\title{
Dorsal root ganglion stimulation for pain relief in painful polyneuropathy
}

Citation for published version (APA):

Koetsier, E. (2020). Dorsal root ganglion stimulation for pain relief in painful polyneuropathy: efficacy and mechanism of action. [Doctoral Thesis, Maastricht University]. ProefschriftMaken. https://doi.org/10.26481/dis.20200902ek

Document status and date:

Published: 01/01/2020

DOI:

10.26481/dis.20200902ek

Document Version:

Publisher's PDF, also known as Version of record

\section{Please check the document version of this publication:}

- A submitted manuscript is the version of the article upon submission and before peer-review. There can be important differences between the submitted version and the official published version of record.

People interested in the research are advised to contact the author for the final version of the publication, or visit the DOI to the publisher's website.

- The final author version and the galley proof are versions of the publication after peer review.

- The final published version features the final layout of the paper including the volume, issue and page numbers.

Link to publication

\footnotetext{
General rights rights.

- You may freely distribute the URL identifying the publication in the public portal. please follow below link for the End User Agreement:

www.umlib.nl/taverne-license

Take down policy

If you believe that this document breaches copyright please contact us at:

repository@maastrichtuniversity.nl

providing details and we will investigate your claim.
}

Copyright and moral rights for the publications made accessible in the public portal are retained by the authors and/or other copyright owners and it is a condition of accessing publications that users recognise and abide by the legal requirements associated with these

- Users may download and print one copy of any publication from the public portal for the purpose of private study or research.

- You may not further distribute the material or use it for any profit-making activity or commercial gain

If the publication is distributed under the terms of Article $25 \mathrm{fa}$ of the Dutch Copyright Act, indicated by the "Taverne" license above, 


\section{Dorsal Root Ganglion Stimulation for Pain Relief in Painful Polyneuropathy: Efficacy and Mechanism of Action}

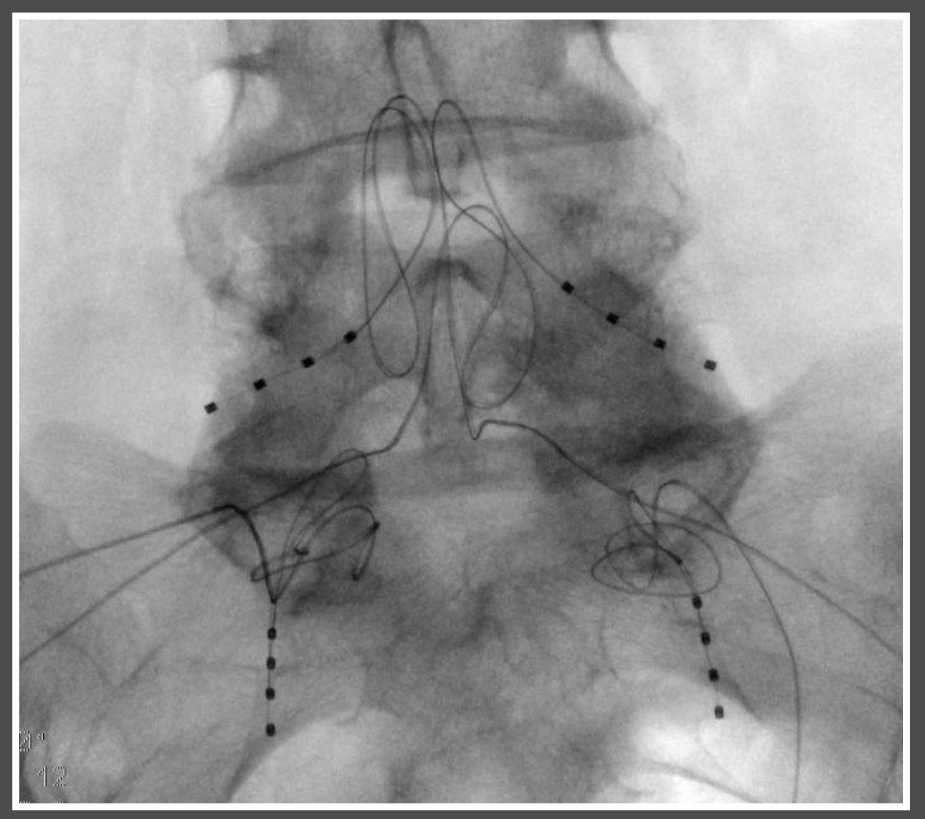

Eva Koetsier 



\title{
Dorsal Root Ganglion Stimulation for Pain Relief in Painful Polyneuropathy:
}

\author{
Efficacy and Mechanism of Action
}


Propositions belonging to the Dissertation

\title{
Dorsal Root Ganglion Stimulation for Pain Relief in Painful Polyneuropathy:
}

\author{
Efficacy and Mechanism of Action
}

By Eva Koetsier

1. Dorsal root ganglion stimulation reduces pain in painful diabetic polyneuropathy (this thesis, Chapters 2, 3)

2. Conventional dorsal root ganglion stimulation and spinal cord stimulation are equally effective for pain relief in painful diabetic polyneuropathy (this thesis, Chapter 4)

3. Dorsal root ganglion stimulation at low frequency is more beneficial for pain relief in painful diabetic polyneuropathy (this thesis, Chapter 5)

4. Dorsal root ganglion stimulation for pain relief in painful diabetic polyneuropathy acts via a different mechanism then spinal cord stimulation (this thesis, Chapter 6)

5. Long-term DRGS-stimulation experiments with use of different stimulation settings are needed to increase translation of the findings to the clinic (this thesis)

6. It is a strange fancy to suppose that science can bring reason to an irrational world, when all it can ever do is give another twist to a normal madness (2002, John N. Gray)

7. The greatest glory in living lies not in never falling, but in rising every time we fall (Nelson Mandela, 1918-2013)

8. Magic is believing in yourself, if you can do that, you can make anything happen (Johann Wolfgang von Goethe, 1749-1832)

9. What the world needs is more women who have quit fearing themselves and started trusting themselves. What the world needs is masses of women who are entirely out of control (2020, Glennon Doyle) 
Copyright (C) Eva Koetsier, 2020

Printing: ProefschriftMaken || www.proefschriftmaken.nl

ISBN 9789463808774

All rights reserved. No part of this publication may be reproduced, stored in a retrieval system or transmitted, in any form or by any means, electronic, mechanical, photocopying, recording or otherwise, without prior permission of the author or the copyright-owning journals for previous published chapters. 


\title{
Dorsal Root Ganglion Stimulation for Pain Relief in Painful Polyneuropathy:
}

\author{
Efficacy and Mechanism of Action
}

\author{
Dissertation \\ to obtain the degree of Doctor at the Maastricht University, \\ on the authority of the Rector Magnificus, \\ Prof.dr. Rianne M. Letschert \\ in accordance with the decision of the Board of Deans, \\ to be defended in public \\ on Wednesday 2 September 2020, at 16:00 hours
}

by

Eva Koetsier 


\section{Supervisors:}

Prof.dr. E.A. Joosten

Prof.dr. J.A.M. van Zundert

\section{Co-supervisor:}

Dr. S.M.J. van Kuijk

\section{Assessment Committee:}

Prof.dr. Y. Temel

Prof.dr. F.J. Huygen

Prof.dr. G. van Koeveringe

Dr. A.L. Liem

Prof.dr. C.D.A. Stehouwer 


\section{TABLE OF CONTENTS}

\section{Chapter 1}

General Introduction and Outline of the Thesis

\section{Chapter 2}

Efficacious Dorsal Root Ganglion Stimulation for Painful Small Fiber Neuropathy:

a Case Report

\section{Chapter 3}

Dorsal Root Ganglion Stimulation for the Management Of Intractable Painful

Polyneuropathy: A prospective open-label, pilot study

\section{Chapter 4}

Effectiveness of Dorsal Root Ganglion Stimulation and Dorsal Column Spinal Cord Stimulation in a Model of Experimental Painful Diabetic Polyneuropathy

\section{Chapter 5}

Dorsal Root Ganglion Stimulation in Experimental Painful Diabetic Polyneuropathy:

Delayed Wash-out of Pain Relief after Low-Frequency $(1 \mathrm{~Hz})$ Stimulation

\section{Chapter 6}

Mechanism of Dorsal Root Ganglion Stimulation for Pain Relief in Painful

Diabetic Polyneuropathy is Not Dependent on GABA Release in the Dorsal Horn

of the Spinal Cord

\section{Chapter 7}

Summary and Discussion

\section{Chapter 8}

Valorization Addendum

Co-author Affiliations

Acknowledgments

About the author

List of Publications 

Chapter 1

General Introduction and Outline of the Thesis 


\section{General Introduction}

Dorsal root ganglion stimulation (DRGS) is an emerging option for treatment of painful polyneuropathy. ${ }^{1,2}$ In section 1 of this General Introduction pain is defined and a description of the different types pain is given. Section 2 goes more into detail about neuropathic pain and its two subcategories central and peripheral neuropathic pain (2.2), followed by an overview of the structure of the peripheral nervous system (2.3). Subsequently, section 3 introduces painful polyneuropathy, and describes the prevalence and typical symptoms (3.1) and primary care management (3.2). In section 4, the neuromodulatory interventions spinal cord stimulation (SCS) (4.1) and DRGS (4.2) in the treatment of painful polyneuropathy are discussed. Additionally, this section give an introduction of stimulation parameters, mechanism of action and experimental research (4.3), and goes then deeper into optimization of stimulation frequency (4.3.1) and the role of gamma-amino-butyric acid (GABA) in the mechanism of action of the analgesic effect of SCS and DRGS (4.3.2). There are several advantages and disadvantages of neuromodulation in animal studies, which are summarized in section 5. Last, section 6 concludes, and presents the aim and the research questions of this thesis.

\section{Pain and Specific Types of Pain}

Pain is a subjective experience and is modulated by past experiences, setting, affect, cognitive influences, gender and cultural expectations. ${ }^{3}$ The International Association for the Study of Pain (IASP) defines pain as an unpleasant sensory and emotional experience associated with actual or potential tissue damage, or described in terms of such damage. ${ }^{4}$ In clinical practice, two types of pain are described: nociceptive pain and neuropathic pain. Nociceptive pain is defined as pain that arises from actual or threatened damage to non-neural tissue. ${ }^{4}$ Nociception is the neural process of encoding noxious stimuli. ${ }^{4}$ It is the process of detection of intense thermal, mechanical, or chemical stimuli by specialized nerve fibers, called nociceptors, that are capable of transducing and encoding the noxious stimuli. ${ }^{4,5}$ Nociceptors are activated in the target organs (skin, muscles, joints and viscera) and convey the nociceptive information to the central nervous system (CNS). ${ }^{3}$ The cell bodies of nociceptors for the body are located in the dorsal root ganglia (DRG), while those for the face in the trigeminal ganglion. Neuropathic pain, on the other hand, is defined as pain caused by a lesion or disease of the somatosensory nervous system. ${ }^{4}$ Neuropathic pain will be discussed more in detail in section 2. A third type of pain, nociplastic pain, defined as pain that arises from altered nociception despite no clear evidence of tissue damage or evidence for lesion or disease of the somatosensory system is recently defined by the IASP but still not implemented in clinical practice. 


\section{Neuropathic Pain}

In the western world, the prevalence of chronic pain varies between 20 and $30 \%$ and about one fifth of these persons has predominantly neuropathic pain. ${ }^{6-9}$ Neuropathic pain is a condition which is characterized by sensory abnormalities such as abnormal unpleasant sensation (dysesthesia), an increased sensitivity to painful stimuli (hyperalgesia) and pain in response to normally innocuous stimuli (allodynia).

The ability to detect noxious stimuli is essential to an organism's survival and serves, initially, as a warning sign to protect the damaged region until it can heal. ${ }^{5}$ However, in chronic neuropathic pain the experience of pain persist after the injury and outlives its usefulness as an acute warning system. ${ }^{5}$ The nervous system is responding then inappropriately to the damage through multiple mechanisms involving the nervous system and its modulators. This results in an unbalanced somatosensory system that misreads sensory inputs and might spontaneously generate painful sensations. . $^{5,10,11}$

\subsection{Central and Peripheral Neuropathic Pain}

Neuropathic pain syndromes can be divided into two general subcategories: those that are consequences of a lesion or disease of the central nervous system and those that are consequences of a lesion or disease of the peripheral nervous system. ${ }^{10,12}$ Central neuropathic pain (CNP) syndromes encompass all disorders that result from a lesion or disease of the nerves within the central nervous system (the brain, the brainstem, or the spinal cord). Common causes of CNP are stroke, multiple sclerosis, and spinal cord injury. ${ }^{13}$ Peripheral neuropathic pain (PNP) syndromes include all disorders that result from a lesion or disease of the nerves within the peripheral nervous system. ${ }^{14}$ PNP syndromes encompass a broad range of disorders affecting the peripheral nervous system, like painful polyneuropathy, radiculopathy, complex regional pain syndrome, postherpetic neuralgia, and trigeminal neuralgia. ${ }^{14}$

\subsection{The Structure of the Peripheral Nervous System}

Peripheral nerves are categorized into motor, sensory, and autonomic nerves. The cell bodies (soma) of motor neurons are located in the ventral gray matter of the spinal cord. The axons of the motor neurons extend to the neuromuscular junction. Sensory neurons are bipolar and have an afferent axon receiving sensory input from the periphery and an efferent axon entering the central nervous system via the dorsal root. The cell body of the sensory neuron resides in the DRG or one of the sensory ganglia of sensory cranial nerves. The DRG is a located bilaterally in the intraforaminal space at each spinal vertebral level. It contains the cell bodies of all the primary sensory neurons innervating the dermatome governed by its spinal segmental level. ${ }^{15}$ The autonomic nervous system is classified into sympathetic and parasympathetic nerves. The neurons of sympathetic nerves are located in the lateral horn of the spinal cord from T1 to L2, whereas the 
neurons of parasympathetic nerves are located in the brain stem and sacral spinal cord $(\mathrm{S} 2, \mathrm{~S} 3$, and $\mathrm{S} 4) .{ }^{16}$

\section{Painful Polyneuropathy}

One of the most common types of peripheral neuropathy is polyneuropathy. ${ }^{14,17}$ In this section painful polyneuropathy is described with focus at its prevalence, typical symptoms (3.1), and primary care management (3.2).

\subsection{Painful Polyneuropathy: Prevalence and Symptoms}

The overall prevalence of polyneuropathy in the general population worldwide ranges from 1 to $3 \%,{ }^{14,18,19}$ and the condition has numerous etiologies of like diabetes mellitus (DM), alcohol abuse, treatment with toxic agents like chemotherapeutic drugs, nutritional deficiencies, autoimmune-mediated causes and hereditary factors, and in 20$50 \%$ of the cases an underlying cause cannot be identified. ${ }^{18,20}$ Polyneuropathy is typically characterized by sensory symptoms in the distal parts of the limbs, such as sensory loss, paresthesia and sharp and shooting pain, with motor disturbances like distal weakness under more severe conditions. ${ }^{14,21-23}$ Symptoms mostly worsen during periods of rest and at night. The most common type of polyneuropathy is distal symmetric polyneuropathy, a diffuse, length-dependent process. ${ }^{14}$ The distal portions of the (longest) nerves projecting to the lower limbs are most often first affected. ${ }^{14}$ When the symptoms progress and the disease reaches more proximal levels of the nerves innervating the lower limbs, also upper limb involvement can follow, with a similar progression proximally starting in the hands. ${ }^{14,24}$ The characteristic distribution and development of pain with painful polyneuropathy is often referred to as a "stocking glove pattern". ${ }^{14}$

Disorders of sensory nerve function are diverse and depend on the type of afferent nerve fibers that are affected. ${ }^{25}$ In small fiber neuropathies (SFN), the medium size myelinated $A_{\delta}$ fibers and the small unmyelinated $C$ fibers are affected. ${ }^{26}$ Diabetic polyneuropathy (DPN) is an example of a predominant SFN. Large fiber neuropathies (LFN), like Friedreich's ataxia, result from dysfunction of the large $A_{\beta}$ fibers that mediate vibratory and touch sensation, ${ }^{23}$ and are characterized by loss of joint position, loss of vibration sense and sensory ataxia. ${ }^{27,28}$ Painful polyneuropathies affect large- and smallnerve fibers and are therefore termed mixed fiber neuropathies (MFN). ${ }^{29,30}$

Neuropathic pain in polyneuropathy is often under-diagnosed and undertreated. It can significantly impair physical and mental quality of life, ${ }^{31,32}$ and has an important socioeconomic impact. ${ }^{9}$ Today, DM is the most common cause of polyneuropathy in the world, accounting for $30-40 \%$ of the cases of polyneuropathy. ${ }^{14,37,41} \mathrm{DM}$ is a worldwide growing epidemic, affecting approximately 380 million people worldwide, and expectedly 438 million by $2030 .{ }^{44}$ Symptoms of DPN occur in up to $50 \%$ of all patients with DM, and up 
to $1 / 3$ of all diabetic patients acquire painful diabetic polyneuropathy (PDPN). ${ }^{45}$ In DPN the small unmyelinated $C$ fibers and thinly myelinated $A_{\delta}$ fibers are primarily targeted early in the disease process, ${ }^{46}$ whereas large myelinated sensory fibers are only affected later in the disease process. ${ }^{42,47}$

\subsection{Painful polyneuropathy: Primary Care Management}

The management of painful polyneuropathy is related to intervene with the underlying cause and etiology. ${ }^{19}$ Examples of treatment of the underlying cause are improved glucose management in case of PDPN and treatment of alcohol dependence in case of alcohol induced polyneuropathy. The treatment of the underlying cause of polyneuropathy needs to be continuously monitored and managed as further progression of the disease and painful neuropathic symptoms occurs and this is often irreversible. First-line drugs in the pain treatment of painful polyneuropathy consist of antidepressants (i.e. tricyclic antidepressants or selective serotonin-norepinephrine reuptake inhibitors), and anticonvulsants (alpha-2- delta agonists). ${ }^{14,42}$ Furthermore, analgesic therapy can be based on the principles of pharmacological pain management as in the World Health Organization (WHO) analgesic ladder for cancer pain relief. ${ }^{48}$ This ladder is divided into three steps. Step One: non-opioids (paracetamol and non-steroidal anti-inflammatory drugs (NSAIDs)). Step Two: weak opioids (e.g. codeine). Step Three: strong opioids (e.g. morphine). 'Adjuvant' analgesics, like antidepressants, anticonvulsants and topicals like lidocaine and capsaicin can be added at any stage of the WHO ladder. ${ }^{48-50}$ Yet, the degree of effectiveness of pharmacological treatment of painful polyneuropathy is often minor and frequently accompanied by unacceptable side effects. ${ }^{51,52}$ Therefore, other (non-pharmacological) treatment options are urgently needed. Emerging options for treatment of painful polyneuropathy are neuromodulatory interventions like SCS and DRGS. ${ }^{1,2,53-56}$ At present, the neuromodulatary interventions SCS and DRGS are considered as a last resort treatment method for patients that suffer from painful polyneuropathy when conventional therapies (e.g., pharmacologic, psychologic, physical treatments) have failed. . $3,55,57,58$

\section{Neuromodulatory interventions}

The International Neuromodulation Society defines therapeutic neuromodulation as "the alteration of nerve activity through targeted delivery of a stimulus, such as electrical stimulation or chemical agents, to specific neurological sites in the body." 59 In the next sections, the neuromodulatory interventions SCS and DRGS will be introduced and their possible mechanisms of action described. 


\subsection{Spinal Cord Stimulation}

SCS is a form of neuromodulation and was first utilized in 1967 and emerged as an application of the Gate Control Theory of Pain of Melzack and Wall. ${ }^{60,61}$ This theory states that electrical stimulation of the large myelinated non-nociceptive $A_{\beta}$-fibers in the dorsal column of the spinal cord activates the inhibitory interneurons to release GABA in the dorsal horn of the spinal cord. This leads to an inhibition of the spinal nociceptive signal from smaller diameter nociceptive $A_{\delta}$ fibers and $C$ fibers to the brain. ${ }^{60,61}$ With SCS, electrodes are epidurally implanted on top of the dorsal columns (fig. 1A and 1B) and connected to a pulse generator for delivery of electrical current to the non-nociceptive $A_{\beta}$ fibers. ${ }^{61} \mathrm{SCS}$ with conventional settings (frequency $20-80 \mathrm{~Hz}$; pulse width 0.2-0.5 ms; amplitude 3.6 to $8.5 \mathrm{~mA})^{62,63}$ has been shown to be effective in patients having a variety of neuropathic pain conditions, including complex regional pain syndrome (CRPS) and failed back surgery syndrome (FBSS). ${ }^{64-65}$ Other study results have suggested its effectiveness for chronic intractable angina pectoris and peripheral vascular diseases. ${ }^{66-}$ ${ }^{68}$ Moreover, SCS has also been shown to be effective in PDPN, ${ }^{53,54,69-75}$ and in intractable painful polyneuropathy due to other causes, like HIV infection and chemotherapy. ${ }^{57,58}$ 


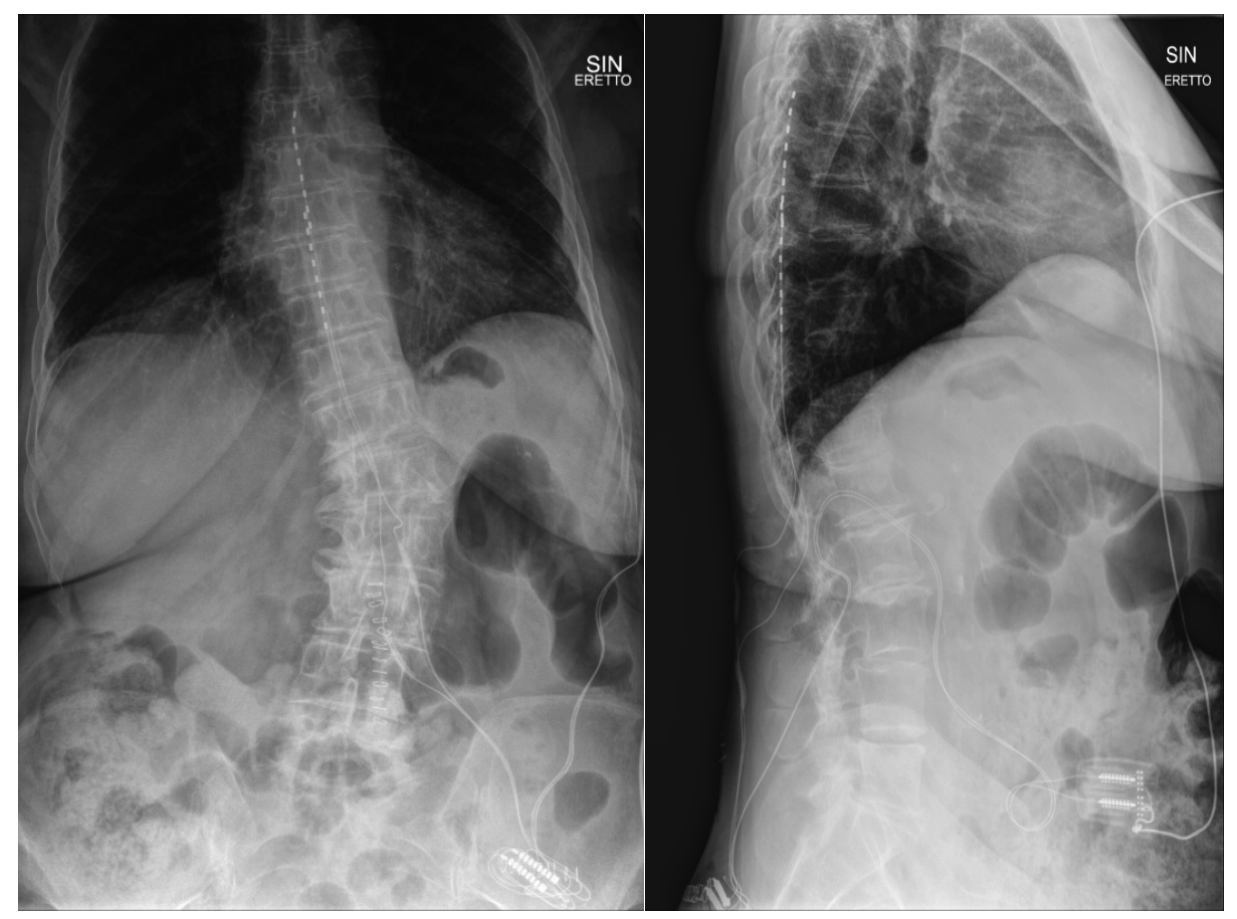

Figure 1. (A) Anterior-posterior fluoroscopic view of two octapolar leads in the epidural space implanted at the Th6-Th10 level of a Failed Back Surgery Syndrome (FBSS) patient with the external pulse generator left-under. (B) Lateral fluoroscopic view of the two octapolar leads dorsally in the epidural space with the external pulse generator right-under.

The precise spinal mechanisms of action of SCS are still unknown and the Gate Control Theory is supposed to only partially explain its pain relieving mechanism. SCS is additionally known to induce orthrodromic activation of a supraspinal network inhibiting the incoming nociceptive signal at spinal levels via descending tracts. ${ }^{61}$ Furthermore, SCS also leads to decreased connectivity between sensory and limbic areas, ${ }^{76}$ resulting in a reduction of the affective component of pain. Moreover, conventional SCS is known to cause peripheral vasodilatation relieving ischemic pain and improving peripheral blood flow. ${ }^{68,77-79}$ Considering this and the vascular mechanisms that are involved in PDPN pathology, it is likely that the SCS-induced improvement of blood perfusion of tissue leads to a better functioning of nervous tissue in PDPN. ${ }^{79,80}$

The majority of literature examining conventional SCS for painful polyneuropathy relates to polyneuropathy caused by DM (PDPN). However, there are limits to the effectiveness of conventional SCS for PDPN, as this treatment is known to provide approximately $50 \%$ pain reduction to only $60 \%$ of PDPN patients. ${ }^{53,69-73,75}$ Forty percent of the patients is therefore not responding to this therapy. Furthermore, the analgesic effect of SCS treatment is known to decrease over time. ${ }^{81}$ Lastly, SCS is often unable to cover the pain in the so-called "difficult-to-reach areas", like the lower extremities (feet) 
and the groin, without generating extensive paresthesias or motor side effects. ${ }^{82,83}$ It is in view of these limitations that new options should be developed and studied. In the last few years, several novel stimulation modalities have already been introduced, including high-frequency (HF) stimulation, ${ }^{84,85}$ burst stimulation, ${ }^{86}$ and also new locations of stimulation have been explored like stimulation of the DRG (DRGS). ${ }^{82}$ In particular the new location of stimulation at the DRG is shown to be a promising new option for treatment of PDPN.

\subsection{Dorsal Root Ganglion Stimulation}

For DRGS, electrodes are implanted directly at the level of the DRG (Fig. 2). ${ }^{87}$ The neurostimulator system (ProclaimTM DRG Neurostimulator System manufactured by St. Jude Medical, now Abbott, Sunnyvale, CA, USA) is comprised of a stimulator device (an external trial neurostimulator [TNS] used for the trial period, followed by an implantable neurostimulator [INS] if successful), up to four quadripolar percutaneous leads and a patient- and clinician-programmer device. The delivery system consists of a flexible lead with a hollow inner lumen and a sheath into which the lead is loaded. A curved stylet is inserted into the lumen of the lead to provide rigidity and directionality to the lead during implantation. Leads are implanted via an epidural needle, according to the technique descripted by Liem et al. ${ }^{82,88}$ Fluoroscopy is used to identify spinal levels for lead placement, to guide lead delivery, and to confirm final lead position. While the patient is under local anesthesia (and lightly sedated), access to the contralateral epidural space is obtained percutaneously using a $14 \mathrm{G}$ needle and applying the loss of resistance technique. The lead is inserted epidurally through the needle in an anterograde fashion and steered into the intraforaminal epidural space above the DRG. Once appropriate lead position is determined, the stylet is partially removed and the lead advanced in the epidural space to create a loop to limit lead migration (Fig. 2). The sheath, needle, and stylet are completely removed and the lead is then anchored to the fascia using tissue anchors. The lead is tunneled to the subcutaneous stimulator pocket, typically in the upper buttocks (or abdomen), and connected to the stimulator device.

As described above (section 2.2, The structure of the peripheral nervous system), the DRG contains the somata for all sensory neuron subtypes, not only for the nonnociceptive $A_{\beta}$ cell bodies but also the $A_{\delta}$ and C-type nociception related cell bodies. While conventional SCS of the dorsal columns can theoretically only directly modulate $A_{\beta}$ fiber signaling, DRGS might therefore also be able to modulate the nociceptive $A_{\delta}$ - and C-type fiber signaling. ${ }^{89}$

Since a DRG innervates a single dermatome of the body, DRGS with conventional settings (frequency 20-70 Hz; pulse width 0.2-0.4 ms; amplitude 800 to $900 \mu \mathrm{A}$ ) 82,90 provides dermatome-specific pain relief. This suggests that DRGS can more precisely target a specific pain area and be more effective for patients with pain that is difficult to target with conventional SCS (e.g. foot and groin pain). ${ }^{15,82,91,92}$ 
DRGS has demonstrated pain relief for groin pain, CRPS and chronic, intractable neuropathic pain of the limbs and trunk. ${ }^{90-93}$ Liem et al. ${ }^{82}$ performed a study investigating the safety and performance of DRGS, which showed clinically significant pain relief after 6 months of treatment in $89 \%$ of the patients suffering from foot pain. Amongst the subjects with foot pain were patients with CRPS, postsurgical pain, radicular pain and pain caused by peripheral nerve damage. Apart from the ability to better reach areas like the feet, DRGS offers several other potential benefits over SCS systems like lack of positional and movement effects on stimulation and reduced migration rate, because of better lead stability within the foramen. ${ }^{22,82,94}$ Additionally, as the anatomical location of the DRG offers a closer proximity to the electrodes compared to the spinal cord and its dorsal columns, reduced power is required. ${ }^{22,82}$

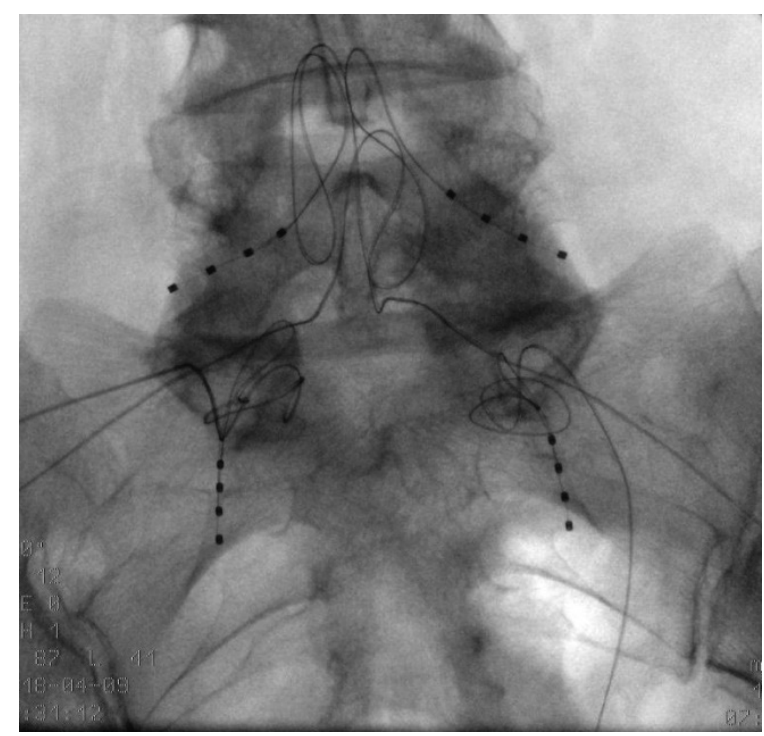

Figure 2. Anterior-posterior fluoroscopic view of two quadripolar leads in the epidural space implanted at the bilateral L5 DRG and at the S1 DRG.

As in painful polyneuropathy pain is mostly present and starts in the lower extremities and as DRGS might cover these difficult-to-reach-areas better, ${ }^{95}$ we hypothesized that DRGS is more effective when compared to SCS. Early findings from one retrospective case series in painful diabetic polyneuropathy patients suggested that DRGS is an effective neuromodulation modality to improve painful symptoms. ${ }^{1}$ Furthermore, the results of another small retrospective case series suggest that DRGS may be an effective treatment option for painful hereditary and idiopathic axonal polyneuropathy. ${ }^{2}$ As only retrospective studies on the effect of DRGS on PDPN have been published as of yet more and prospective clinical evidence is warranted to further confirm the efficacy of this treatment. 


\subsection{Stimulation Parameters, Mechanism of Action and Experimental Research}

Experimental research on animals is considered as an important part of translational research. Understanding of the working mechanism underlying SCS- and DRGS-induced pain relief may enable optimization of treatment and result in better treatment outcomes. Most of our knowledge about the mechanisms underlying the pain-relieving effect of SCS and, more recently DRGS, is derived from experiments performed in rodents. ${ }^{96,97}$ Initially, experiments were performed on nerve lesioned rats, usually a partial lesion of the sciatic nerve or a complete section of its peripheral braches or spinal root, leading to an increased sensitivity ("hypersensitivity") to normally innocuous mechanical stimuli..${ }^{96,98}$ The rats were then implanted with a miniaturized SCS system and it was demonstrated that SCS suppressed or attenuated the hypersensitivity. ${ }^{96,99,100}$ To determine "pain" in animals, the gold standard is the use of von Frey filaments and paw withdrawal response. The procedure of the von Frey test is such that a series of filaments of different weights are applied in a standardized and protocolized sequence to the plantar surface of the hind paw of the animal with a pressure that causes the filament to bend. ${ }^{101}$ Mechanical hypersensitivity in these animals is suggested to mimic allodynia in humans, and this behavioral sign is considered to represent "pain" ${ }^{96}$

SCS and its effect on pain relief (paw withdrawal) in diabetic rats with PDPNassociated-mechanical-hypersensitivity has been described in female Sprague-Dawley rats $^{79,102,103}$ DM was induced in these rats with a single intraperitoneal injection of 65 $\mathrm{mg} / \mathrm{kg}$ streptozotocin (STZ). Four days after STZ injection, blood glucose level was determined in blood derived from the saphenous vein of the leg. Rats with a glucose level of $\geq 15 \mathrm{mmol} / \mathrm{L}$ were considered diabetic, ${ }^{101}$ and included into the studies. Animals were tested for mechanical hypersensitivity using von Frey paw withdrawal testing. ${ }^{104}$

DRGS has also been used and studied in an animal model. ${ }^{97}$ With use of monopolar electrodes which were placed around the L5-DRG in rats with chronic peripheral neuropathic pain a significant effect on mechanical hypersensitivity was noted. This study made it possible to analyze the mechanism of action underlying DRGS in chronic neuropathic pain. ${ }^{97}$ In the present thesis we further developed the technique of DRGS in rats and extended it to a model of PDPN. The mechanism of action of DRGS in pain relief of PDPN in relation to use of various stimulation settings like frequency, intensity and pulse width of stimulation can now be studied. However, attention should be paid to the fact that these variables and stimulation parameters are tightly related and interact to modulate pain pathways within the nervous system. ${ }^{62}$ The combination of the various stimulation parameters results in a "total charge delivery" and the manner in which stimulation parameters are combined is important for investigating mechanisms of action and the subsequent outcomes. ${ }^{62}$ In the next section, special attention will be paid to the analgesic effect of frequency of stimulation. 


\subsubsection{Optimization of Stimulation Frequency}

Optimization of the stimulation frequency might result in additional beneficial effects in painful polyneuropathy, and PDPN in particular. It is known that after an action potential, the sodium channels of a neuron are temporarily inactivated. The period of this inactivation is called the "refractory period" and the duration of this period is inversely related to the fiber diameter. Consequently, large diameter fibers are able to follow higher stimulation rates, whereas the activation of smaller will be desynchronized. ${ }^{105}$ From this can be concluded that for modulation of the smaller nociceptive As fibers and $C$ fibers lower frequencies are needed as compared to large $A_{\beta}$-fibers. As with DRGS not only the $A_{\beta}$ fiber signaling can be modulated, but probably also the nociceptive $A_{\delta}$ - and C-type fiber signaling, ${ }^{89}$ lower frequency settings might be more effective for DRGS as compared to SCS. Nevertheless, until today it is not known with which frequency the smaller diameter fibers can be stimulated and as it is known that extracellular stimulation preferentially activates large-diameter myelinated axons over small unmyelinated axons, ${ }^{106}$ the smaller diameter fibers may not be stimulated by DRGS at all. ${ }^{25}$

Conventionally, SCS and DRGS frequencies vary between 20 and $80 \mathrm{~Hz} .62,63,82,90,107$ High-frequency SCS varies between 1 and $10 \mathrm{kHz}$, and has been shown to attenuate hyperalgesia in a rat chronic peripherally induced neuropathic model to a greater extent than stimulation at $50 \mathrm{~Hz} .{ }^{108}$ The same may hold true for PDPN, but the pathophysiology of PDPN also includes the vasculature system and here SCS has been suggested to not only modulate the nerve fibers but additionally to induce vasodilation. ${ }^{109}$ Previous studies have shown that SCS at higher frequencies significantly increased cutaneous blood flow in comparison to lower frequencies. ${ }^{110,111}$ On the other hand, the results of the experimental study of Pluijms et al., ${ }^{102}$ testing the effect of different SCS frequencies on pain relief in PDPN-animals, showed that the effect of SCS on mechanical hypersensitivity is equal when applied at low- $(4.0-10 \mathrm{~Hz})$, mid- $(35-55 \mathrm{~Hz})$ and higher- $(150-375 \mathrm{~Hz})$ frequencies (pulse width $0.2 \mathrm{~ms}$; amplitude at $67 \%$ of motor threshold $(0.19 \pm 0.01 \mathrm{~mA})$ ). Furthermore, van Beek et al., ${ }^{103}$ compared the efficacy of low- $(5 \mathrm{~Hz})-$, mid- $(50 \mathrm{~Hz})$, and higher- $(500 \mathrm{~Hz})$ frequency on mechanical hypersensitivity in chronic experimental PDPN. A delayed but not superior effect of the higher frequency on mechanical hypersensitivity in the chronic PDPN animals compared to lower frequencies was noted. Van Beek et al. recently demonstrated that long-term SCS (of four weeks, 12 hours/day) with a frequency of $50 \mathrm{~Hz}$ resulted in decreased baseline mechanical hypersensitivity in a rat model of PDPN, suggesting an improvement in the baseline condition with long-term SCS treatment. ${ }^{79}$ Concluding, frequency seems to be important in pain relieving effect in PDPN, but optimal frequency still needs to be determined. Future experiments should be performed with longer-term DRGS-stimulation paradigms which much more closely mimics the clinical situation.

With respect to DRGS and pain relieving effect in PDPN, there is little data about the effect of different stimulation frequencies. As one might expect that frequency also with 
DRGS will be an important stimulation parameter in establishing optimal pain relief, further clinical and experimental research is warranted.

\subsubsection{Analgesic Effect of SCS or DRGS: Mechanism of Action and a Role of GABA}

Experimental studies have demonstrated that peripheral nerve injury can induce a dysfunction of the (natural) GABA-ergic inhibition and a neuronal hyperexcitability in the spinal dorsal horn. ${ }^{112-116}$ This dysfunction of the GABA-ergic inhibition and the neuronal hyperexcitability are supposed to be among the major underlying causes of neuropathic pain. ${ }^{112-116}$ The theory that nerve injury induces a loss in GABA-ergic inhibition in the spinal dorsal horn, causing neuropathic pain, is sustained by experimental studies that have shown that pharmacological antagonism of GABA-ergic inhibition either via the $G_{A B A}$, but mainly via the $G A B A_{B}$ receptor in the spinal cord induced mechanical hypersensivity. ${ }^{117-119}$ The stimulation of the $A_{\beta}$ fibers with SCS seems to interrupt these processes as it induces an activation of the GABA-ergic inhibitory interneurons in the dorsal horn and subsequently an inactivation of the wide dynamic range (WDR) neurons, thereby impairing pain transmission to higher brain centers. ${ }^{60,105,120}$ Studies in nerve lesioned rats demonstrated that SCS indeed increases the spinal dorsal horn GABA release. ${ }^{116,121,122}$ The role of GABA in the analgesic effect of SCS has been confirmed by the fact that SCS-induced increases in spinal dorsal horn extracellular GABA levels were only observed in rats receiving adequate pain relief from SCS ("responders-toSCS"). $^{116,121,122}$ Additionally, the results of an experimental study of Janssen et al. ${ }^{123}$ demonstrated that responders-to-SCS showed decreased levels of intracellular GABAimmunoreactivity in the spinal dorsal horn in comparison to non-responders-to-SCS and sham-SCS animals. Therefore, a relation between the release of intracellular accumulated GABA in the spinal cord dorsal horn and the analgesic effect of SCS was hypothesized. ${ }^{114,123-125}$ Animal experiments furthermore demonstrated that intrathecal administration of a $\mathrm{GABA}_{B}$ receptor agonist (baclofen) enhances the analgesic effect of SCS, and even converts SCS non-responding animals into good responders. ${ }^{126}$ These experimental findings were also translated to the clinic. ${ }^{127-129}$ In a study of Lind et al. ${ }^{127}$ with patients suffering from neuropathic pain of peripheral origin responding poorly to SCS, 7 out of 48 patients experienced a significant increase of the effect of SCS when baclofen was administered intrathecally. These patients were implanted with pumps for intrathecal baclofen delivery together with SCS and the enhanced analgesic effect persisted on the long-term. ${ }^{128}$

The mechanisms underlying DRGS and its ensuing pain relief are as yet unknown, and it is likely that it shares some spinal and supraspinal mechanisms with SCS, dealing with the $A_{\beta}$ fibers activation with both types of stimulation. Therefore, it is likely that the analgesic effect of DRGS is linked to activation of GABA release in the dorsal horn, likewise SCS. However, it is on the other hand recently postulated that the modulation of a GABAmediated Gate Control mechanism with DRGS actually takes place in a primary Gate for nociceptive control, namely at the DRG itself, instead of at the secondary level of the 
dorsal horn. This hypothesis is supported by an experimental study of Du at al., ${ }^{130}$ which showed that key components of the GABA-ergic transmission are expressed in the DRG. Their study demonstrated that depolarizing stimuli induce GABA release in the DRG and at the same time a reduction of the neuronal excitability in the DRG in response to GABA was observed. ${ }^{130}$ Furthermore, focal infusion of GABA, GABAA agonists, and GABA reuptake inhibitors to the $D R G$ resulted in alleviating neuropathic pain. The delivery of $G A B A_{A}$ receptor antagonists to the DRG on the other hand exacerbated peripherally induced nociception. ${ }^{130}$ These results taken altogether indicate that there is a endogenous GABA-ergic control in the DRG, ${ }^{131,132}$ and analgesic effects of focally applied GABA mimetics suggest that DRGS acts via the modulation of a GABA mediated Gate Control at the level of the DRG itself. This does not exclude, however, that DRGS, like SCS, induces also an increased GABA release at the level of the dorsal horn. In view of these findings it is important to study the effect of DRGS in relation to its effect on GABA-release both at the spinal dorsal horn and at the DRG as this may shed light on the underlying mechanism of this type of stimulation as compared to SCS.

\section{Advantages and Disadvantages of Neuromodulation in Animal Studies}

There are several discrepancies between animal and human studies as with SCS and/or DRGS. Examples of these discrepancies are the way of assessing pain (von Frey in animals (a reflex based outcome) measure vs. questionnaires in humans, which also take into account affective components of pain), the size of anatomical structures such as the DRG, and the time frame of the PDPN animal model and the stimulation paradigm used (shortterm stimulation in our PDPN animal model, long-term stimulation in PDPN patients). Nevertheless, there are several distinct advantages of neuromodulation animal studies. Animal studies can be conducted in a highly standardized manner (DRGS paradigm, housing, routine, age, sex, duration of pain), while clinical studies have to deal with a large amount intra- and inter-subject variability. For example, patients are often allowed to make changes within their stimulation paradigm (like changes in amplitude) throughout the day, within a limited range. ${ }^{75,88,92}$ In animal studies, the experimental design can be designed in such a way that for example only the applied DRGS frequency differs between the different animal groups. Highly standardized research is pivotal for acquiring objective results. 


\section{Conclusion, Aim of Thesis and Research Questions}

Based on the first clinical data, DRGS is considered an interesting option for treatment of painful polyneuropathy and further studies are needed to establish the effect of DRGS in PDPN. To improve the effectiveness of DRGS in treatment of PDPN, a mechanism based approach is needed and therefore experimental studies are required. The development of an animal model for DRGS in PDPN is a first next step. Then insights into effects of various stimulation parameters either combined with cellular-molecular studies on the mechanism underlying DRGS will add to our understanding and result in development of optimizing the analgesic effect of DRGS.

The main objectives of the research as presented in this thesis are:

Clinical research

- $\quad$ assess effectiveness of DRGS in patients with painful polyneuropathy

\section{Experimental research}

- develop an experimental model for DRGS in PDPN and test pain relieving effect of DRGS as compared to SCS

- $\quad$ study the effect of DRGS frequency and pain relieving effect in an animal model of PDPN

- $\quad$ study the mechanism of DRGS in pain relief in PDPN animals focused at role of GABA in the spinal dorsal horn

\subsection{Aim of the Thesis}

The aim of this thesis is to establish and improve the effectiveness of DRGS and pain relief in painful polyneuropathy. This is not based on clinical studies only, but also based on development of an animal model of DRGS in PDPN and understanding the underlying mechanism. With use of an animal model we aim to increase effectiveness of this therapy per se, and as compared to SCS, and to further understand the underlying mechanism of DRGS in pain relief. 


\subsection{Research Questions}

In view of this aim, the following research questions (RQs) were formulated:

RQ1: Does DRGS result in pain relief in patients with painful polyneuropathy?

RQ2A: Is it possible to develop an animal model for DRGS in PDPN?

RQ2B: Is DRGS more effective in pain relief as compared to SCS in this animal model?

RQ3: IS DRGS frequency an important parameter in pain relief in an animal model of PDPN?

RQ4: Is spinal GABA release involved in the mechanism underlying DRGS and pain relief in an animal model of PDPN?

\section{Outline of the Thesis}

The research presented in this thesis was part of a collaboration between the following centers:

- The Pain Management Center, Neurocenter of Southern Switzerland, Regional Hospital of Lugano, Switzerland

- The Department of Anesthesiology and Pain Management, MUMC +, Maastricht, The Netherlands

- The Department of Anesthesiology and Pain Management, VU University Medical Center, Amsterdam, The Netherlands

$R Q 1$ is addressed in Chapters $\mathbf{2}$ and $\mathbf{3}$. In comparison to SCS, DRGS is known to better cover difficult-to-reach areas like the foot. ${ }^{82,91,92}$ In Chapter $\mathbf{2}$ we describe a first case of intractable painful SFN of the foot, successfully treated with DRGS of the left L5 DRG. Results from this case report demonstrate that the DRG is a promising neural stimulation target to treat neuropathic pain due to intractable SFN. Furthermore, the results of two recently published retrospective case series suggest that DRGS is an effective treatment option for painful diabetic, hereditary and idiopathic polyneuropathy. ${ }^{1,2}$ Nevertheless, prospective studies are warranted to confirm the efficacy of this treatment as a treatment option for painful polyneuropathy. Therefore, the aim of the study presented in Chapter $\mathbf{3}$ was to obtain preliminary prospective information on the ability of DRGS to reduce pain intensity in patients with intractable painful polyneuropathy. Secondary objectives in this study were to assess impact of DRGS on the patients' global impression of change, physical functioning, quality of life and mood. 
$R Q 2 A$ and $2 B$ are addressed in Chapter 4. To obtain further insight into the efficacy underlying the pain relieving effect of DRGS in polyneuropathy, in particular in PDPN, we implemented DRGS in an already operational and meticulously tested PDPN animal model. ${ }^{102}$ The development of the animal model for DRGS in PDPN allowed to compare the pain relieving effect of DRGS to SCS in PDPN associated mechanical hypersensitivity in STZ-induced diabetic rats (Chapter 4).

$R Q 3$ is addressed in Chapter 5. The study presented in this chapter was aimed to compare the pain relieving effect of DRGS at various frequencies: $1 \mathrm{~Hz}$ (defined as "low-frequency", LF), at $20 \mathrm{~Hz}$ (defined as "mid-frequency", MF), at $1000 \mathrm{~Hz}$ (defined as "high-frequency", HF) and sham-DRGS in an animal model of PDPN.

$R Q 4$ is addressed in Chapter 6. The mechanisms underlying DRGS and its ensuing pain relief are as yet unknown, and it is likely that it shares some spinal and supraspinal mechanisms with SCS, dependent on $A_{\beta}$ fibers activated by both types of stimulation. The study presented in Chapter 6 was aimed to investigate the hypothesis that DRGS, like SCS, induces GABA release from spinal dorsal horn cells. 


\section{References}

1. Eldabe S, Espinet A, Wahlstedt A, et al. Retrospective case series on the treatment of painful diabetic peripheral neuropathy with dorsal root ganglion stimulation. Neuromodulation. 2018;21:787-792.

2. Ho KWD, Rempe T, Jerath N, Antony A. Dorsal root ganglion stimulation as a potentially effective treatment for painful hereditary and idiopathic axonal polyneuropathy: a retrospective case series. Neuromodulation. 2020;23:234-238.

3. Gold MS, Gebhart GF. Nociceptor sensitization in pain pathogenesis. Nat Med. 2010;16:1248-1257.

4. IASP Terminology. https://www.iasp-pain.org/terminology?navltemNumber=576\#Pain (accessed March 7, 2020).

5. Basbaum Al, Bautista DM, Scherrer G, Julius D. Cellular and molecular mechanisms of pain. Cell. 2009;139:267-284.

6. Cohen SP, Mao J. Neuropathic pain: mechanisms and their clinical implications. BMJ. 2014;348:f7656.

7. Torrance N, Smith BH, Bennett MI, Lee AJ. The epidemiology of chronic pain of predominantly neuropathic origin. Results From a General Population Survey. J Pain. 2006;7:281-289.

8. Bouhassira D, Lantéri-Minet M, Attal N, Laurent B, Touboul C. Prevalence of chronic pain with neuropathic characteristics in the general population. Pain. 2008;136:380-387.

9. Breivik H, Collett B, Ventafridda V, Cohen R, Gallacher D. Survey of chronic pain in Europe: prevalence, impact on daily life, and treatment. Eur J Pain. 2006;10:287-333.

10. Meacham K, Shepherd A, Mohapatra DP, Haroutounian S. Neuropathic pain:central vs. peripheral mechanisms. Curr Pain Headache Rep. 2017; 21:28.

11. Braz J, Solorzano C, Wang X, Basbaum Al. Transmitting pain and itch messages: a contemporary view of the spinal cord circuits that generate gate control. Neuron. 2014;82:522-536.

12. Haanpää M, Attal N, Backonja M, et al. NeuPSIG guidelines on neuropathic pain assessment. Pain. 2011;152:14-27.

13. Watson JC, Sandroni P. Central neuropathic pain syndromes. Mayo Clinic Proceedings. 2016; 91:372-385.

14. Callaghan BC, Price RS, Feldman EL. Distal symmetric polyneuropathy a review. JAMA. 2015;314:21722181.

15. Graham RD, Bruns TM, Duan B, Lempka SF. Dorsal root ganglion stimulation for chronic pain modulates Aß-fiber activity but not C-fiber activity: A computational modeling study. Clin Neurophysiol. 2019;130:941-951.

16. Chung T, Prasad K, Lloyd TE. Peripheral neuropathy. Clinical and electrophysiological considerations. Neuroimaging Clin N Am. 2014;24:49-65.

17. Tavee J, Zhou L. Small fiber neuropathy: A burning problem. Cleve Clin J Med. 2009;76:297-305.

18. Hanewinckel R, van Oijen M, Ikram MA, van Doorn PA. The epidemiology and risk factors of chronic polyneuropathy. Eur J Epidemiol. 2016;31:5-20.

19. Watson JC, Dyck PJ. Peripheral Neuropathy: A practical approach to diagnosis and symptom management. Mayo Clin Proc. 2015 ;90:940-951.

20. Bennett DL, Woods CG. Painful and painless channelopathies. Lancet Neurol. 2014;13:587-599.

21. Andreassen CS, Jakobsen J, Andersen H. Muscle weakness: a progressive late complication in diabetic distal symmetric polyneuropathy. Diabetes. $2006 ; 55: 806-812$.

22. Tesfaye S, Selvarajah D. Advances in the epidemiology, pathogenesis and management of diabetic peripheral neuropathy. Diabetes Metab Res Rev. 2012;28(SUPPL. 1):8-14.

23. Gwathmey KG. Sensory polyneuropathies. Continuum. 2017;23:1411-1436.

24. Tesfaye S, Boulton AJ, Dickenson AH. Mechanisms and management of diabetic painful distal symmetrical polyneuropathy. Diabetes Care. 2013;36:2456-2465.

25. Gwathmey KG, Pearson KT. Diagnosis and management of sensory polyneuropathy. BMJ. 2019;365:I1108.

26. Terkelsen AJ, Karlsson P, Lauria G, Freeman R, Finnerup NB, Jensen TS. The diagnostic challenge of small fibre neuropathy: clinical presentations, evaluations, and causes. Lancet Neurol. 2017;16:934-944.

27. Siao P, Kaku M. A Clinician's Approach to Peripheral neuropathy. Semin Neurol. 2019;39:519-530. 


\section{Chapter 1}

28. Martinez AR, Nunes MB, Nucci A, França MC Jr. Sensory neuronopathy and autoimmune diseases. Autoimmune Dis. 2012;2012:873587.

29. Hansen N, Kahn AK, Zeller D, Katsarava Z, Sommer C, Üçeyler N. Amplitudes of pain-related evoked potentials are useful to detect small fiber involvement in painful mixed fiber neuropathies in addition to quantitative sensory testing - An electrophysiological study. Front Neurol. 2015 7;6:244.

30. Singleton JR, Smith AG, Bromberg MB. Painful sensory polyneuropathy associated with impaired glucose tolerance. Muscle and Nerve. 2001;24:1225-1228.

31. Aslam A, Singh J, Rajbhandari SM. The impact of painful diabetic neuropathy on quality of life: An observational study. Diabetes Prim Care. 2014;16: 212-219.

32. Van Acker K, Bouhassira D, De Bacquer D, et al. Prevalence and impact on quality of life of peripheral neuropathy with or without neuropathic pain in type 1 and type 2 diabetic patients attending hospital outpatients clinics. Diabetes Metab. 2009;35:206-213.

33. England JD, Asbury AK. Peripheral neuropathy. Lancet. 2004;363:2151-2161.

34. Burns TM, Mauermann ML. The evaluation of polyneuropathies. Neurology. 2011;76(7 Suppl 2):S6-13.

35. Peltier A, Goutman SA, Callaghan BC. Painful diabetic neuropathy. BMJ. 2014;348:g1799.

36. Mygland $\AA$, Monstad P. Chronic polyneuropathies in Vest-Agder, Norway. Eur J Neurol. 2001;8:157-165.

37. Visser NA, Notermans NC, Linssen RS, Van Den Berg LH, Vrancken AF. Incidence of polyneuropathy in Utrecht, the Netherlands. Neurology. 201;84:259-264.

38. George J, Twomey JA. Causes of polyneuropathy in the elderly. Age Ageing. 1986;15:247-249.

39. Lin KP, Kwan SY, Chen SY, et al. Generalized neuropathy in Taiwan: an etiologic survey. Neuroepidemiology. 1993;12:257-261.

40. Johannsen L, Smith T, Havsager AM, et al. Evaluation of patients with symptoms suggestive of chronic polyneuropathy. J Clin Neuromuscul Dis. 2001;3:47-52.

41. Rudolph T, Farbu E. Hospital-referred polyneuropathies-causes, prevalences, clinical- and neurophysiological findings. Eur J Neurol. 2007;14:603-608.

42. Li Y. Axonal sensorimotor polyneuropathies. Continuum Lifelong Learn Neurol. 2017;23:1378-1393.

43. Tesfaye S, Boulton AJM, Dyck PJ, et al. Diabetic neuropathies: update on definitions, diagnostic criteria, estimation of severity, and treatments. Diabetes Care. 2010;33:2285-2293.

44. Shaw JE, Sicree RA, Zimmet PZ. Global estimates of the prevalence of diabetes for 2010 and 2030. Diabetes Res Clin Pract. 2010;87:4-14.

45. Abbott CA, Malik RA, Van Ross ERE, Kulkarni J, Boulton AJM. Prevalence and characteristics of painful diabetic neuropathy in a large community-based diabetic population in the U.K. Diabetes Care. 2011;34:2220-2224.

46. Løseth S, Stålberg E, Jorde R, Mellgren SI. Early diabetic neuropathy: thermal thresholds and intraepidermal nerve fibre density in patients with normal nerve conduction studies. J Neurol. 2008 ;255:1197-1202.

47. Said G, Baudoin D, Toyooka K. Sensory loss, pains, motor deficit and axonal regeneration in lengthdependent diabetic polyneuropathy. J Neurol. 2008;255:1693-1702.

48. https://www.who.int/cancer/palliative/painladder/en/ (accessed January 27, 2020).

49. Laird B, Colvin L, Fallon M. Management of cancer pain: basic principles and neuropathic cancer pain. Eur J Cancer. 2008 ;44:1078-1082.

50. Jensen TS, Madsen CS, Finnerup NB. Pharmacology and treatment of neuropathic pains. Curr Opin Neurol. $2009 ; 22: 467-474$

51. Bril V, England J, Franklin GM, et al. Evidence-based guideline: treatment of painful diabetic neuropathy. PM\&R. 2011;3:345-352.

52. Sindrup SH, Jensen TS. Pharmacologic treatment of pain in polyneuropathy. Neurology. 2000;55:915-920.

53. Slangen R, Schaper NC, Faber CG, et al. Spinal cord stimulation and pain relief in painful diabetic peripheral neuropathy: a prospective two-center randomized controlled trial. Diabetes Care. 2014;37:3016-3024.

54. van Beek M, Slangen R, Schaper NC, et al. Sustained treatment effect of spinal cord stimulation in painful diabetic peripheral neuropathy: 24-month follow-up of a prospective two-center randomized controlled trial. Diabetes Care. 2015;38:e132-134. 
55. de Vos CC, Meier K, Zaalberg PB, et al. Spinal cord stimulation in patients with painful diabetic neuropathy: A multicentre randomized clinical trial. Pain. 2014;155:2426-2431.

56. van Beek M, Geurts JW, Slangen R, et al. Severity of neuropathy is associated with long-term spinal cord stimulation outcome in painful diabetic peripheral neuropathy: Five-year follow-up of a prospective twocenter clinical trial. Diabetes Care. 2018;41:32-38.

57. Abd-Elsayed A, Schiavoni N, Sachdeva H. Efficacy of spinal cord stimulators in treating peripheral neuropathy: a case series. J Clin Anesth. 2016;28:74-77.

58. Knezevic NN, Candido KD, Rana S, Knezevic I. The use of spinal cord neuromodulation in the management of HIV-Related Polyneuropathy. Pain Physician. 2015;18:E643-650.

59. International Neuromodulation Society. Neuromodulation, or Neuromodulatory Effect. https://www.neuromodulation.com/neuromodulation-defined (Accessed September 13, 2019).

60. Melzack R, Wall P. Pain mechanisms: a new theory. Science. 1965;150:971-979.

61. Linderoth B, Foreman RD. Conventional and novel spinal stimulation algorithms: hypothetical mechanisms of action and comments on outcomes. Neuromodulation. 2017;20:525-533.

62. Miller JP, Eldabe S, Buchser E, Johanek LM, Guan Y, Linderoth B. Parameters of spinal cord stimulation and their role in electrical charge delivery: a review. Neuromodulation. 2016;19:373-384.

63. Geurts JW, Joosten EA, Van Kleef M. Current status and future perspectives of spinal cord stimulation in treatment of chronic pain. Pain. 2017;158:771-774.

64. Kemler MA, Barendse GA, van Kleef $M$, et al. Spinal cord stimulation in patients with chronic reflex sympathetic dystrophy. N Engl J Med. 2000;343:618-624.

65. Kumar K, Taylor RS, Jacques L, et al. Spinal cord stimulation versus conventional medical management for neuropathic pain: A multicentre randomised controlled trial in patients with failed back surgery syndrome. Pain. 2007;132:179-188.

66. Tsigaridas N, Naka K, Tsapogas P, Pelechas E, Damigos D. Spinal cord stimulation in refractory angina. A systematic review of randomized controlled trials. Acta Cardiol. 2015;70:233-243.

67. Eldabe S, Thomson S, Duarte R, et al. The effectiveness and cost-effectiveness of spinal cord stimulation for refractory angina (RASCAL study): a pilot randomized controlled trial. Neuromodulation. 201 ;19(1):60-70.

68. Ubbink DT, Vermeulen H. Spinal cord stimulation for non-reconstructable chronic critical leg ischaemia. Cochrane Database Syst Rev. 2013;(2):CD004001.

69. Kumar K, Toth C, Nath RK. Spinal cord stimulation for chronic pain in peripheral neuropathy. Surg Neurol. 1996;46:363-369.

70. Tesfaye S, Watt J, Benbow SJ, Pang KA, Miles J, MacFarlane IA. Electrical spinal-cord stimulation for painful diabetic peripheral neuropathy. Lancet. 1996;348:1698-1701.

71. Daousi C, Benbow SJ, MacFarlane IA. Electrical spinal cord stimulation in the long-term treatment of chronic painful diabetic neuropathy. Diabet Med. 2005;22:393-398.

72. de Vos CC, Rajan V, Steenbergen W, van der Aa HE, Buschman HP. Effect and safety of spinal cord stimulation for treatment of chronic pain caused by diabetic neuropathy. J Diabetes Complicat. 2009;23:40-45.

73. Pluijms WA, Slangen $\mathrm{R}$, Bakkers $\mathrm{M}$, et al. Pain relief and quality-of-life improvement after spinal cord stimulation in painful diabetic polyneuropathy: A pilot study. Br J Anaesth. 2012;109:623-629.

74. Slangen R, Pluijms WA, Faber CG, Dirksen CD, Kessels AGH, Van Kleef M. Sustained effect of spinal cord stimulation on pain and quality of life in painful diabetic peripheral neuropathy. $\mathrm{Br} J$ Anaesth. 2013;111:1030-1031.

75. Vos C, Meier K, Zaalberg P, et al. Spinal cord stimulation in patients with painful diabetic neuropathy: a multicentre randomized clinical trial. Pain. 2014;155:2426-2431.

76. Deogaonkar M, Sharma M, Oluigbo C, et al. Spinal cord stimulation (SCS) and runctional magnetic resonance imaging (fMRI): modulation of cortical connectivity with therapeutic SCS. Neuromodulation. 2016;19:142-153.

77. Ubbink DT, Vermeulen H. Spinal cord stimulation for critical leg ischemia: a review of effectiveness and optimal patient selection. J Pain Symptom Manage. 2006;31(4 Suppl):S30-35. 


\section{Chapter 1}

78. Deer TR, Mekhail N, Provenzano D, et al. The appropriate use of neurostimulation of the spinal cord and peripheral nervous system for the treatment of chronic pain and ischemic diseases: The neuromodulation appropriateness consensus committee. Neuromodulation. 2014;17:515-550.

79. van Beek M, Hermes D, Honig WM, et al. Long-term spinal cord stimulation alleviates mechanical hypersensitivity and increases peripheral cutaneous blood perfusion in experimental painful diabetic polyneuropathy. Neuromodulation. 2018;21:472-479.

80. van Beek M. Thesis: Spinal Cord Stimulation in Clinical and experimental painful diabetic polyneuropathy. Maastricht University. 2017

81. van Beek M, Geurts JW, Slangen R, et al. Severity of neuropathy is associated with long-term spinal cord stimulation outcome in painful diabetic peripheral neuropathy: Five-year follow-up of a prospective twocenter clinical trial. Diabetes Care. 2018;41:32-38.

82. Liem L, Russo M, Huygen FJPM, et al. A multicenter, prospective trial to assess the safety and performance of the spinal modulation dorsal root ganglion neurostimulator system in the treatment of chronic pain. Neuromodulation. 2013:471-482.

83. Verrills P, Sinclair C, Barnard A. A review of spinal cord stimulation systems for chronic pain. J Pain Res. 2016;9:481-492.

84. Kapural L, Yu C, Doust MW, et al. Novel 10-kHz high-frequency therapy (HF10 Therapy) is superior to traditional low-frequency spinal cord stimulation for the treatment of chronic back and leg pain. Anesthesiology. 2015;123:851-860.

85. Kapural L, Yu C, Doust MW, et al. Comparison of 10-kHz high-frequency and traditional low-frequency spinal cord stimulation for the treatment of chronic back and leg pain: 24-month results from a multicenter, randomized, controlled pivotal trial. Neurosurgery. 2016 ;79:667-677.

86. de Ridder D, Plazier M, Kamerling N, Menovsky T, Vanneste S. Burst spinal cord stimulation for limb and back pain. World Neurosurg. 2013 ;80:642-649.

87. Deer TR, Krames E, Mekhail N, et al. The appropriate use of neurostimulation: new and evolving neurostimulation therapies and applicable treatment for chronic pain and selected disease states. Neuromodulation. 2014;17:599-615.

88. Liem L, Russo M, Huygen FJPM, et al. One-year outcomes of spinal cord stimulation of the dorsal root ganglion in the treatment of chronic neuropathic pain. Neuromodulation. 2015;18:41-49.

89. Koopmeiners AS, Mueller S, Kramer J, Hogan QH. Effect of electrical field stimulation on dorsal root ganglion neuronal function. Neuromodulation. 2013;16:304-311; discussion 310-311.

90. Deer TR, Grigsby E, Weiner RL, Wilcosky B, Kramer JM. A prospective study of dorsal root ganglion stimulation for the relief of chronic pain. Neuromodulation. 2013;16:67-72.

91. Schu S, Gulve A, Eldabe S, et al. Spinal cord stimulation of the dorsal root ganglion for groin pain-a retrospective review. Pain Pract. 2015;15:293-299.

92. Deer TR, Levy RM, Kramer J, et al. Dorsal root ganglion stimulation yielded higher treatment success rate for complex regional pain syndrome and causalgia at 3 and 12 months: a randomized comparative trial. Pain. 2017;158:669-681.

93. van Buyten JP, Smet I, Liem L, Russo M, Huygen F. Stimulation of dorsal root ganglia for the management of complex regional pain syndrome: a prospective case series. Pain Pract. 2014;15:208-216.

94. Chang Chien GC, Mekhail N. Alternate intraspinal targets for spinal cord stimulation: a systematic review. Neuromodulation. 2017;20:629-641.

95. Galer BS, Gianas A, Jensen MP. Painful diabetic polyneuropathy: epidemiology, pain description, and quality of life. Diabetes Res Clin Pract. 2000;47:123-128

96. Ultenius C, Song Z, Lin P, Meyerson BA, Linderoth B. Spinal GABAergic mechanisms in the effects of spinal cord stimulation in a rodent model of neuropathic pain: Is GABA synthesis involved? Neuromodulation. 2013;16:114-120.

97. Pan B, Yu H, Fischer GJ, Kramer JM, Hogan QH. Dorsal root ganglionic field stimulation relieves spontaneous and induced neuropathic pain in rats. J Pain. 2016;17:1349-1358.

98. Linderoth B, Meyerson BA. Spinal cord stimulation: Exploration of the physiological basis of a widely used therapy. Anesthesiology. $2010 ; 113: 1265-1267$. 
99. Cui JG, Meyerson BA, Sollevi A, Linderoth B. Effect of spinal cord stimulation on tactile hypersensitivity in mononeuropathic rats is potentiated by simultaneous $G A B A(B)$ and adenosine receptor activation. Neurosci Lett. 1998;247:183-186.

100. Li D, Yang H, Meyerson BA, Linderoth B. Response to spinal cord stimulation in variants of the spared nerve injury pain model. Neurosci Lett. 2006;400:115-120.

101. Calcutt NA. Modeling diabetic sensory neuropathy in rats. Methods Mol Med. 2004;99:55-65.

102. Pluijms WA, van Kleef M, Honig WM, Janssen SP, Joosten EA. The effect of spinal cord stimulation frequency in experimental painful diabetic polyneuropathy. Eur J Pain. 2013;17:1338-1346.

103. van Beek M, Kleef M, Linderoth $B$, et al. Spinal cord stimulation in experimental chronic painful diabetic polyneuropathy: Delayed effect of High-frequency stimulation. Eur J Pain. 2017;21:795-803.

104. Chaplan SR, Bach FW, Pogrel JW, Chung JM, Yaksh TL. Quantitative assessment of tactile allodynia in the rat paw. J Neurosci Methods. 1994;53:55-63.

105. Smits H, van Kleef M, Holsheimer J, Joosten EA. Experimental spinal cord stimulation and neuropathic pain: mechanism of action, technical aspects, and effectiveness. Pain Pract. 2013;13:154-168.

106. Rattay F. Analysis of models for external stimulation of axons. IEEE Trans Biomed Eng. 1986;33:974-977.

107. Deer TR, Levy RM, Kramer J, et al. Dorsal root ganglion stimulation yielded higher treatment success rate for CRPS and causalgia at 3 and 12 months. Pain. 2017;158:669-681.

108. Shechter R, Yang F, Xu Q, et al. Conventional and kilohertz-frequency spinal cord stimulation produces intensity-and frequency-dependent inhibition of mechanical hypersensitivity in a rat model of neuropathic pain. Anesthesiology. 2013;119:422-432.

109. Wu M, Thorkilsen MM, Qin C, Farber JP, Linderoth B, Foreman RD. Effects of spinal cord stimulation on peripheral blood circulation in rats with streptozotocin-induced diabetes. Neuromodulation. 2007;10:216223.

110. Gao J, Wu M, Li L, et al. Effects of spinal cord stimulation with "standard clinical" and higher frequencies on peripheral blood flow in rats. Brain Res. 2010;1313:53-61.

111. Kao CH, Chen JJ, Hsu YM, Bau DT, Yao CH, Chen YS. High-frequency electrical stimulation can be a complementary therapy to promote nerve regeneration in diabetic rats. PLoS One. 2013;8:e79078.

112. Patel S, Naeem S, Kesingland A, et al. The effects of GABA(B) agonists and gabapentin on mechanical hyperalgesia in models of neuropathic and inflammatory pain in the rat. Pain. 2001;90:217-226.

113. Somers DL, Clemente FR. Dorsal horn synaptosomal content of aspartate, glutamate, glycine and GABA are differentially altered following chronic constriction injury to the rat sciatic nerve. Neurosci Lett. 2002;323:171-174.

114. Moore KA, Kohno T, Karchewski LA, Scholz J, Baba H, Woolf CJ. Partial peripheral nerve injury promotes a selective loss of GABAergic inhibition in the superficial dorsal horn of the spinal cord. J Neurosci. 2002;22:6724-6731.

115. Drew GM, Siddall PJ, Duggan AW. Mechanical allodynia following contusion injury of the rat spinal cord is associated with loss of GABAergic inhibition in the dorsal horn. Pain. 2004;109:379-388.

116. Stiller CO, Cui JG, O'Connor WT, Brodin E, Meyerson BA, Linderoth B. Release of $Y$-aminobutyric acid in the dorsal horn and suppression of tactile allodynia by spinal cord stimulation in mononeuropathic rats. Neurosurgery. 1996;39:367-375.

117. Hao JX, Xu XJ, Wiesenfeld-Hallin Z. Intrathecal $\gamma$-aminobutyric acidB (GABAB) receptor antagonist CGP 35348 induces hypersensitivity to mechanical stimuli in the rat. Neurosci Lett. 1994;182:299-302.

118. Malan TP, Mata HP, Porreca F. Spinal GABA(A) and GABA(B) receptor pharmacology in a rat model of neuropathic pain. Anesthesiology. 2002;96:1161-1167.

119. Sivilotti L, Woolf CJ. The contribution of GABAA and glycine receptors to central sensitization: disinhibition and touch-evoked allodynia in the spinal cord. J Neurophysiol. 1994;72:169-179.

120. Guan Y. Spinal cord stimulation: Neurophysiological and neurochemical mechanisms of action. Curr Pain Headache Rep. 2012;16:217-225.

121. Cui JG, O'Connor WT, Ungerstedt U, Linderoth B, Meyerson BA. Spinal cord stimulation attenuates augmented dorsal horn release of excitatory amino acids in mononeuropathy via a GABAergic mechanism. Pain. 1997;73:87-95. 


\section{Chapter 1}

122. Linderoth B, Stiller CO, Gunasekera L, O'Connor WT, Ungerstedt U, Brodin E. Gamma-aminobutyric acid is released in the dorsal horn by electrical spinal cord stimulation: an in vivo microdialysis study in the rat. Neurosurgery. 1994;34:484-488; discussion 488-489.

123. Janssen SP, Gerard S, Raijmakers ME, Truin M, Van Kleef M, Joosten EA. Decreased intracellular GABA levels contribute to spinal cord stimulation-induced analgesia in rats suffering from painful peripheral neuropathy: the role of KCC2 and GABAAreceptor-mediated inhibition. Neurochem Int. 2012;60:21-30.

124. Eaton MJ, Plunkett JA, Karmally S, Martinez MA, Montanez K. Changes in GAD- and GABAimmunoreactivity in the spinal dorsal horn after peripheral nerve injury and promotion of recovery by lumbar transplant of immortalized serotonergic precursors. J Chem Neuroanat. 1998;16:57-72.

125. Kim J, Kim SJ, Lee H, Chang JW. Effective neuropathic pain relief through sciatic nerve administration of GAD65-expressing rAAV2. Biochem Biophys Res Commun. 2009;388:73-78.

126. Cui JG, Linderoth B, Meyerson BA. Effects of spinal cord stimulation on touch-evoked allodynia involve GABAergic mechanisms. An experimental study in the mononeuropathic rat. Pain. 1996;66:287-295.

127. Lind G, Meyerson BA, Winter J, Linderoth B. Intrathecal baclofen as adjuvant therapy to enhance the effect of spinal cord stimulation in neuropathic pain: a pilot study. Eur J Pain. 2004;8:377-383.

128. Lind G, Schechtmann G, Winter J, Meyerson BA, Linderoth B. Baclofen-enhanced spinal cord stimulation and intrathecal baclofen alone for neuropathic pain: long-term outcome of a pilot study. Eur J PAIN. 2008;12:132-136.

129. Schechtmann G, Lind G, Winter J, Meyerson BA, Linderoth B. Intrathecal clonidine and baclofen enhance the pain-relieving effect of spinal cord stimulation: a comparative placebo-controlled, randomized trial. Neurosurgery. 2010;67:173-181.

130. Du X, Hao H, Yang Y, et al. Local GABAergic signaling within sensory ganglia controls peripheral nociceptive transmission. J Clin Invest. 2017;127:1741-1756.

131. Obradovic AL, Scarpa J, Osuru HP, et al. Silencing the $\alpha 2$ subunit of $\gamma$-aminobutyric acid type A receptors in rat dorsal root ganglia reveals its major role in antinociception posttraumatic nerve injury. Anesthesiology. 2015;123:654-667.

132. Naik AK, Pathirathna S, Jevtovic-Todorovic V. GABAA receptor modulation in dorsal root ganglia in vivo affects chronic pain after nerve injury. Neuroscience. 2008;154:1539-1553. 


\section{Chapter}

\section{Efficacious Dorsal Root Ganglion Stimulation for Painful Small Fiber Neuropathy: a Case Report}

Paolo Maino $\mathrm{MD}^{1}$, Eva Koetsier $\mathrm{MD}^{1}$, Alain Kaelin² PhD, MD, Claudio Gobbi MD³ ${ }^{3}$, Roberto S.G.M. Perez PhD ${ }^{4}$

${ }^{1}$ Pain Management Center, Neurocenter of Southern Switzerland, Ospedale Regionale di Lugano, Switzerland;

${ }^{2}$ Department of Neuroscience, Neurocenter of Southern Switzerland, Ospedale Regionale di Lugano, Switzerland;

${ }^{3}$ Department of Neurology, Neurocenter of Southern Switzerland, Ospedale Regionale di Lugano, Switzerland;

${ }^{4}$ Department of Anesthesiology, VU University Medical Center, Amsterdam, The Netherlands.

Pain Physician, 2017;20:E459-E463. 


\section{Abstract}

Small fiber neuropathy is a disorder of the peripheral nerves which typical symptoms are burning, sharp and shooting pain and sensory disturbances in the feet. Pain treatment depends principally on the underlying etiology with concurrent administration of antidepressants, anticonvulsants, opioids, and topical treatments like capsaicin and local anesthetics. However, treatments for pain relief in these patients frequently fail. We describe the first case of intractable painful small fiber neuropathy of the foot, successfully treated with spinal cord stimulation of the left L5 dorsal root ganglion.

A 74-year-old man presented at our clinic with severe intractable pain, dysesthesia and allodynia of the left foot caused by idiopathic small fiber neuropathy, confirmed by skin biopsy. His pain score was 8 on a standard 0-10 numeric rating scale. As the pain was not satisfactory controlled by conventional therapy, dorsal root ganglion stimulation was proposed to the patient and, after informed consent, a specifically designed percutaneous stimulation lead was placed over the left L5 dorsal root ganglion and connected to an external neurostimulator. After a positive trial of 10 days, a permanent neurostimulator was implanted. Twenty months post-implantation the patient continued to experience that the stimulation-induced paresthesia covered the entire pain area and reported a pain rating of 4 .

Results from this case report demonstrate that the dorsal root ganglion is a promising neural stimulation target to treat neuropathic pain due to intractable small fiber neuropathy. Prospective controlled studies are warranted to confirm the efficacy of this treatment as a treatment option for the aforementioned condition. 


\section{Introduction}

Small fiber neuropathy (SFN) is a disorder of the peripheral nerves that affects thinly myelinated $A \delta$ and unmyelinated, nociceptive $C$ nerve fibers. Typical symptoms affect the lower limbs in a distal-to-proximal gradient and are burning, sharp and shooting pain in the feet, sensory disturbances, and in some cases autonomic dysfunction. Symptoms mostly worsen during periods of rest and at night. ${ }^{1}$ SFN might be acquired secondary to a variety of diseases including diabetes mellitus, HIV infection, or chemotherapy. However, in up to $50 \%$ of cases of painful SFN, an underlying cause cannot be identified. ${ }^{2}$ The diagnosis of SFN is often challenging because the small fibers are undetectable to routine neurophysiological nerve conduction studies. Tests such as quantitative sensory testing (QST) and laser Doppler flowmetry may help to confirm the diagnosis. Biopsy at the ankle showing reduced intraepidermal nerve fiber (IENF) density is however the most reliable technique to diagnose SFN to date. ${ }^{3}$ Pain treatment depends principally on the underlying etiology with concurrent administration of antidepressants, anticonvulsants, opioids, and topical treatments like capsaicin and local anesthetics. However, treatments for pain relief in these patients frequently fail. ${ }^{1}$

Spinal cord stimulation (SCS) is used to treat chronic, intractable neuropathic pain when conventional therapies have failed and has been shown to be effective in patients having a variety of neuropathic pain conditions, including complex regional pain syndrome (CRPS), failed back surgery syndrome (FBSS), and diabetic polyneuropathy. ${ }^{4-6}$ Dorsal root ganglion stimulation (DRGS) is a relatively new mode of SCS with selective stimulation in specific dermatomes and has demonstrated neuropathic pain relief for groin pain, CRPS and pain of the limbs and trunk. ${ }^{7-10}$ DRGS may offer several potential benefits over conventional SCS, including lack of postural effects, lower therapeutic power demands, and selective stimulation in the dermatomes and therefore the ability to reach areas like the feet that are typically difficult to reach using conventional SCS. ${ }^{7}$ In this report we describe a first case of successful treatment of intractable painful SFN with left L5 DRGS. The patient gave permission to publish this case report. 


\section{Case report}

A 74-old male (retired pilot) presented at our clinic with burning and shooting pain in his feet that had progressively worsened in the past 6 years. The pain was predominantly localized on the dorsal side of his left foot and he experienced allodynia, hypoesthesia, and dysesthesia in the same region. The pain increased especially at rest and at night, which led to sleep disturbances. The patient reported a pain score of 8 out of 10 at rest and a pain score of 2-3 during the day on a standard numeric rating scale (NRS, $0=$ no pain; 10 = extremely painful).

The neurological examination was normal. His medical history was significant for hyperlipidemia, left common carotid artery stenosis, coronary artery disease and a depressive disorder. His medications included oral daily doses of aspirin $100 \mathrm{mg}$ and atorvastatin $20 \mathrm{mg}$. Atorvastatin, because of its known potential side effects of myositis and myalgia, was stopped for two weeks but the symptoms did not improve. In 2010, lumbar MRI demonstrated a mild spinal stenosis without radicular compression. Electromyographic assessment performed in 2010 and 2011 showed no signs of sensitive or motoric polyneuropathy of the large fibers. In 2011, the patient scored 7 out of 21 on the Hospital Anxiety and Depression Scale (HADS), which is considered normal. ${ }^{11}$ QST performed in 2011 according to the protocol of the German Research Network on Neuropathic Pain, ${ }^{12}$ revealed dysesthesia, thermal hypoesthesia and hypoalgesia to pinprick on the left foot and an increased vibration detection threshold. Laser Doppler flowmetry did not show any flare response as expression of disturbed C-fiber function and blood tests revealed normal full blood count, renal and thyroid function, glucose levels, ferritin, folic acid and vitamin B12. Other serologies, including antineutrophil cytoplasmic antibody, antinuclear antibody, hepatitis C, human immunodeficiency virus (HIV), and paraneoplastic antibodies were negative. Finally, vascular disturbances of the leg were excluded and in March 2014 a biopsy of the skin confirmed the diagnosis of SFN via pathological reduction of intraepidermal unmyelinated nerve fibers. There was no history of SFN in his family.

Over the course of six years the pain of his previously-undiagnosed SFN was neither satisfactorily controlled by optimized doses of multiple medications including gabapentin, pregabalin, duloxetine, amitriptyline, mirtazapine, lidocaine patches, topical capsaicin $8 \%$, and cannabis, nor by treatment with a transcutaneous electrical nerve stimulation (TENS), physical therapy, acupuncture, and a corticosteroid infiltration at the level of the 1 st ray of the left foot. Only ice packs and pressure applied on his left foot provided some relief at night.

As the pain was not satisfactory controlled by conventional therapy, neuromodulation was considered. DRGS was chosen because of its known selective stimulation in the dermatomes and therefore the ability to reach areas like the feet that are typically difficult to reach using conventional SCS. Informed consent was obtained. The left L5 dorsal root ganglion (DRG) was chosen as the pain was predominantly felt in the dorsal 
side of the patient's left foot. On April 7, 2014 a quadripolar DRG stimulation lead (Axium $^{\text {TM }}$ Neurostimulator System, Spinal Modulation, Inc., Menlo Park, CA, U.S.A.) was placed percutaneously according the procedure described by Liem et al. ${ }^{7}$ Access to the contralateral epidural space was achieved using a 14-gauge, 10-cm Tuohy needle applying a loss of resistance technique. The lead was advanced under fluoroscopic guidance in an anterograde fashion and steered into the medial aspect of the left L5/S1 intervertebral foramen over the left L5 DRG (Fig 1).

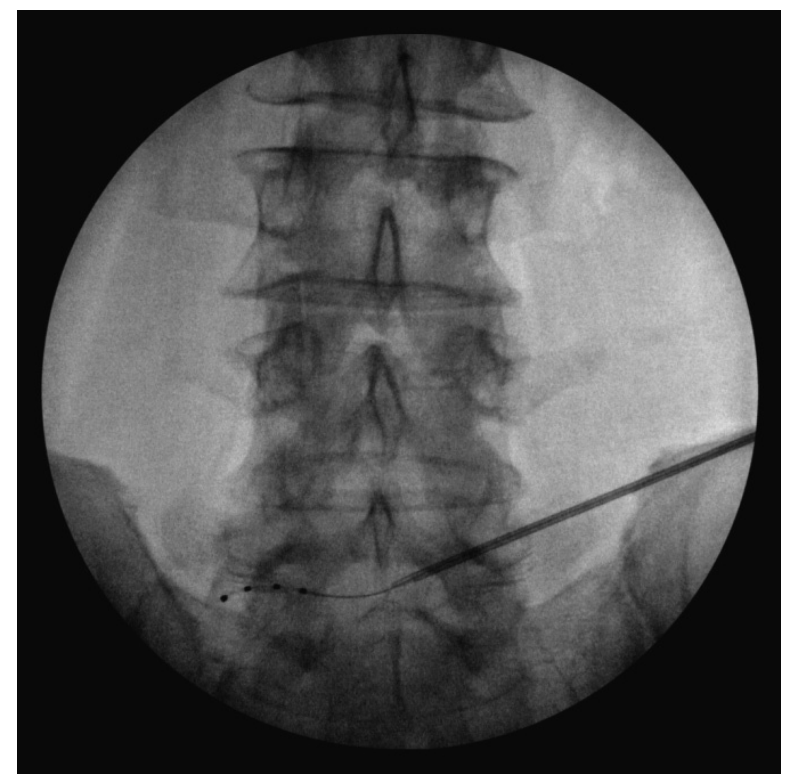

Figure 1. Lead placement at the left L5 DRG

The lead was connected to an external trial neurostimulator, stimulation was initiated, and stimulation-induced paresthesia covered the entire pain area on the dorsal site of the left foot. During 10 days of trial period, the patient reported a pain score of 3 out of 10 on the NRS at rest (an improvement of 62.5\%). Having completed a successful trial, the implantable neurostimulator was placed in the patient's abdominal wall (pulse width: $670 \mu \mathrm{s}$; frequency: $40 \mathrm{~Hz}$; amplitude: $1.88 \mathrm{~mA}$ ). Two months post-implantation, the patient still experienced complete pain coverage, graded his pain as 2 out of 10 on the NRS during stimulation (an improvement of 75\% from baseline), and improved on all domains of the McGill Pain Questionnaire (MPQ). Also the degree of disability, as measured by the Oswestry Disability Index (ODI), reduced substantially (Table 1). In addition, the patient reported that his sleep quality had improved and that he did no longer need to apply ice packs on his left foot to alleviate the pain before going to bed. To describe his feelings, the patient stated "now it's paradise". The device was used 24 hours a day. The patient reported resumption of pain within minutes when the 
neurostimulator was switched off. At the sixth month follow-up the patient still reported a pain rating of NRS 2 and at the twelfth and the twentieth month follow-up he reported a pain rating of 4 . The augmentation in NRS of two points could possibly be explained by progression of the SFN. However, the patient was completely satisfied with the pain relief from stimulation, did not need additional pain treatment and reported his Global Impression of Change (GIC) on all the above mentioned time points as "much improved". The improvement on the MPQ and the degree of disability measured by the ODI, remained stable.

Table 1. Pain and function are improved with DRGS.

\begin{tabular}{|c|c|c|c|c|c|c|}
\hline & & \multirow[t]{2}{*}{ Baseline } & \multicolumn{2}{|c|}{2 Month Follow- 6 Month } & \multirow{2}{*}{$\begin{array}{l}1 \text { year } \\
\text { Follow-up }\end{array}$} & \multirow{2}{*}{$\begin{array}{l}20 \text { Month } \\
\text { Follow up }\end{array}$} \\
\hline & & & up & Follow-up & & \\
\hline \multicolumn{2}{|c|}{ Numeric Rating Scale (NRS) } & 8 & 2 & 2 & 4 & 4 \\
\hline \multicolumn{7}{|l|}{ McGill Pain } \\
\hline Questionnaire & Total Pain & 2.55 & 1.45 & & 1.05 & 1.09 \\
\hline \multirow{4}{*}{ (MPQ) Scores } & Continuous & 2.83 & 1.67 & & 0.83 & 0.83 \\
\hline & Intermittent & 1.33 & 0.83 & & 0 & 0.67 \\
\hline & Neuropathic & 3.67 & 2.00 & & 2.5 & 1.83 \\
\hline & Affective & 2.25 & 1.25 & & 2 & 1.5 \\
\hline \multicolumn{7}{|c|}{ Oswestry Disability Index (ODI) } \\
\hline & & $32 \%$ & $8 \%$ & & $12 \%$ & $6 \%$ \\
\hline & & (Moderate & (Minimum & & & \\
\hline & & Disability) & Disability) & & & \\
\hline
\end{tabular}

\section{Discussion}

This is the first description of a successful case of intractable painful SFN treated with DRGS. The DRG is located between the dorsal root and the spinal nerve within the neural foramen and is composed of cell bodies of primary sensory neurons. It transduces pain to the central nervous system and pathological changes in the DRG are described during chronic neuropathic pain states. ${ }^{13}$ Furthermore, it has recently been reported that mutations in sodium channels expressed in DRG neurons can induce hyperexcitability that contribute to the pathophysiology of painful small-fiber neuropathy. ${ }^{14}$ DRGS reduces excitability of sensory neuronal somata in the DRG in vitro, which suggests that it provides an analgesic effect by reducing somatic excitability. ${ }^{15}$ This may underlie the beneficial analgesic effects that have been observed in our patient.

Although a case report is a report related to the care of an individual patient, it could be a source of new ideas in medicine, guide the treatments in clinical practice, and generate hypotheses for future clinical studies. To date, our patient remains satisfied with the DRGS and has not experienced any disturbances or complications related to the neurostimulator. We realize that this is a single case, but we consider the present results to be encouraging and the DRG to be a potential new neural stimulation target for 
reducing chronic intractable neuropathic pain due to SFN of the lower limbs. Keeping in mind appropriate patient selection and indication assessment, DRGS can be part of an overall treatment plan to manage difficult to treat neuropathic pain syndromes when conventional therapies have failed. Furthermore, we would like to remind the readers that SFN mostly affects the feet bilaterally, ${ }^{1}$ and although it is feasible to apply DRGS bilaterally as the neurostimulator can support up to four leads, the case made in this report applies to predominantly unilateral pain related to SFN. Prospective controlled studies (for both unilateral and bilateral SFN related pain) are needed to evaluate the efficacy of DRGS as a treatment option in SFN. 


\section{References}

1. Hovaguimian A, Gibbons CH. Diagnosis and treatment of pain in small-fiber neuropathy. Curr Pain Headache Rep 2011; 15:193-200.

2. Bennett DL, Woods CG. Painful and painless channelopathies. Lancet Neurol 2014; 13:587-599.

3. Lauria G, Lombardi R. Skin biopsy in painful and immune-mediated neuropathies. J Peripher Nerv Syst 2012; 17 Suppl 3:38-45.

4. Kemler MA, Barendse GA, van Kleef M, de Vet HC, Rijks CP, Furnée CA, van den Wildenberg FA. Spinal cord stimulation in patients with chronic reflex sympathetic dystrophy. N Eng/ J Med 2000; 343:618624.

5. Kumar K, Taylor RS, Jacques L, Eldabe S, Meglio M, Molet J, Thomson S, O'Callaghan J, Eisenberg E, Milbouw G, Buchser E, Fortini G, Richardson J, North RB. Spinal cord stimulation versus conventional medical management for neuropathic pain: a multicentre randomised controlled trial in patients with failed back surgery syndrome. Pain 2007; 132:179-188.

6. Slangen R, Pluijms WA, Faber CG, Dirksen CD, Kessels AG, van Kleef M. Sustained effect of spinal cord stimulation on pain and quality of life in painful diabetic peripheral neuropathy. Br J Anaesth 2013; 111:1030-1031.

7. Liem L, Russo M, Huygen F J, Van Buyten JP, Smet I, Verrills P, Cousins M, Brooker C, Levy R, Deer T, Kramer J. A multicenter, prospective trial to assess the safety and performance of the spinal modulation dorsal root ganglion neurostimulator system in the treatment of chronic pain. Neuromodulation 2013; 16:471-482.

8. Schu S, Gulve A, Eldabe S, Baranidharan G, Wolf K, Demmel W, Rasche D, Sharma M, Klase D, Jahnichen G, Wahlstedt A, Nijhuis H, Liem L. Spinal cord stimulation of the dorsal root ganglion for groin pain - a retrospective review. Pain Pract 2015; 15:293-299.

9. Van Buyten JP, Smet I, Liem L, Russo M, Huygen F. Stimulation of dorsal root ganglia for the management of complex regional pain syndrome: a prospective case series. Pain Pract 2015; 15:208216.

10. Deer TR, Grigsby E, Weiner RL, Wilcosky B, Kramer JM. A prospective study of dorsal root ganglion stimulation for the relief of chronic pain. Neuromodulation 2013; 16:67-71.

11. Zigmond AS, Snaith RP. The hospital anxiety and depression scale. Acta Psychiatr Scand 1983; 67:361-370.

12. Rolke R, Magerl W, Campbell KA, Schalber C, Caspari S, Birklein F, Treede RD. Quantitative sensory testing: a comprehensive protocol for clinical trials. Eur J Pain 2006; 10:77-88.

13. Sapunar D, Kostic S, Banozic A, Puljak L. Dorsal root ganglion - a potential new therapeutic target for neuropathic pain. J Pain Res 2012; 5:31-38.

14. Han C, Vasylyev D, Macala LJ, Gerrits MM, Hoeijmakers JG, Bekelaar KJ, Dib-Hajj SD, Faber CG, Merkies IS, Waxman SG. The G1662S NaV1.8 mutation in small fibre neuropathy: impaired inactivation underlying DRG neuron hyperexcitability. J Neurol Neurosurg Psychiatry 2014; 85:499505.

15. Koopmeiners AS, Mueller S, Kramer J, Hogan QH. Effect of electrical field stimulation on dorsal root ganglion. 


\section{chapter 3}

\section{Dorsal Root Ganglion Stimulation for the Management of Intractable Painful Polyneuropathy: A prospective open-label, pilot study}

E. Koetsier, ${ }^{1,2^{*}}$ S.M.J. van Kuijk, ${ }^{3}$ G. Melli, ${ }^{4,5,6}$ J. Dukanac, ${ }^{1}$ M. Barbero, ${ }^{1,7}$ J. van Zundert, ${ }^{8,9}$ E.A. Joosten, ${ }^{8,10}$ P. Maino ${ }^{1,2}$

${ }^{1}$ Pain Management Center, Neurocenter of Southern Switzerland, Lugano, 6962, Switzerland

${ }^{2}$ Division of Anaesthesiology, Department of Acute Medicine, Regional Hospital of Lugano, Lugano, 6900, Switzerland

${ }^{3}$ Department of Clinical Epidemiology and Medical Technology Assessment, Maastricht University Medical Center+, Maastricht, 6202 AZ, the Netherlands

${ }^{4}$ Laboratory for Biomedical Neurosciences, Neurocenter of Southern Switzerland, Torricella-Taverne, 6807, Switzerland

${ }^{5}$ Faculty of Biomedical Sciences, Università della Svizzera Italiana, Lugano, 6900, Switzerland

${ }^{6}$ Neurology Department, Neurocenter of Southern Switzerland, Regional Hospital of Lugano, Lugano, 6900, Switzerland

${ }^{7}$ Rehabilitation Research Laboratory 2rLab, Department of Business Economics, Health and Social Care, University of Applied Sciences and Arts of Southern Switzerland, Landquart, Manno/Landquart, 6928, Switzerland

${ }^{8}$ Department of Anesthesiology and Pain Management, Maastricht University Medical Center+, Maastricht, 6202 AZ, The Netherlands

${ }^{9}$ Department of Anesthesiology and Multidisciplinary Pain Centre, Ziekenhuis OostLimburg, Genk/Lanaken, 3600, Belgium

${ }^{10}$ Department of Translational Neuroscience, School of Mental Health and Neuroscience (MHeNS), University of Maastricht, Maastricht, 6229 ER, The Netherlands

Submitted 


\section{Abstract}

Objectives: Dorsal root ganglion stimulation (DRGS) is a promising neurostimulation modality in the treatment of intractable painful polyneuropathy. The aim of this prospective pilot study was to investigate the effect of DRGS on pain intensity in patients with painful intractable polyneuropathy.

Methods: Nine patients with chronic, intractable painful polyneuropathy in the lower limbs were recruited. In each subject, between two and four quadripolar percutaneous DRGS leads were placed in the lateral epidural space at the level of the L5 and S1 dorsal root ganglion. If trial stimulation was successful, defined as a reduction of $\geq 50 \%$ in daytime and/or night-time pain intensity, a definitive implantable pulse generator (IPG) was implanted. Pain intensity was scored using an 11-point numeric rating scale. Additionally, patients' global impression of change (PGIC), pain extent, presence of neuropathic pain, physical functioning, health related quality of life, and mood were assessed.

Results: Eight out of 9 patients had a successful trial phase, of which 7 received an IPG. Six out of the 7 implanted patients showed stable treatment success and an improvement on the PGIC during the follow up period of 6 months. Pain extent and impact of pain on functioning improved significantly during follow up period.

Discussion: The results of this study suggest that DRGS reduces significantly both pain intensity and PGIC in patients with refractory painful polyneuropathy in the lower extremities. Large-scale clinical trials are needed to prove the efficacy of DRGS in intractable pain associated with polyneuropathy. 


\section{Introduction}

The worldwide prevalence of polyneuropathy in the general population ranges from 1 to $3 \%$ and rises to $7 \%$ in the elderly. ${ }^{1}$ In the western world, diabetes mellitus (DM) is the cause of polyneuropathy in around $30-40 \%$ of cases. ${ }^{2-5}$ Polyneuropathy can also be acquired secondary to a variety of other conditions including alcohol abuse, vitamin $\mathrm{B}_{12}$ deficiency, human immunodeficiency virus (HIV) infection, chronic inflammatory demyelinating polyneuropathy (CIDP), and after chemotherapy. ${ }^{2,6,7}$ Furthermore, in up to $50 \%$ of patients with polyneuropathy an underlying cause cannot be identified. ${ }^{8}$ One of the most limiting symptoms of polyneuropathy is neuropathic pain, which is present in up to $1 / 3$ of the patients. ${ }^{2,9,10}$ Although pain in polyneuropathy is known to significantly impair health-related quality of life (HRQoL) and a high socio-economic impact, ${ }^{11}$ it is often underdiagnosed and undertreated. ${ }^{2}$

The efficacy of pharmacological treatment including antidepressants (i.e., tricyclic antidepressants or selective serotonin-norepinephrine reuptake inhibitors), and anticonvulsants (alpha-2- delta agonists) is limited, and treatment compliance is often hindered by unacceptable side effects. ${ }^{12,13}$ Therefore, other (non-pharmacological) treatment options are needed. Spinal cord stimulation (SCS ${ }^{14-19}$ and dorsal root ganglion stimulation (DRGS) ${ }^{18-21}$ are emerging neurostimulation last resort treatment options for painful polyneuropathy, when conventional therapies have failed. SCS has been shown to be effective for pain treatment in complex regional pain syndrome (CRPS), failed back surgery syndrome (FBSS), chronic intractable angina pectoris and peripheral vascular diseases. ${ }^{22-26}$ Moreover, SCS has also been demonstrated to be effective in painful diabetic polyneuropathy (PDPN) $)^{14,18,27-34}$ and case reports suggest that this treatment is also effective in intractable painful polyneuropathy due to other causes, like HIV infection and chemotherapy. ${ }^{20,21}$ Nevertheless, conventional SCS often provides incomplete pain relief in PDPN patients, and in around $40 \%$ of these patients SCS does not lead to a clinically relevant pain relief. ${ }^{14,18,19}$ With SCS, focal areas of pain such as the feet, which are often first affected in these patients, are difficult to target. ${ }^{35,36}$ In comparison to SCS, DRGS is known to better cover the "difficult-to-reach areas" like the feet and the groin. ${ }^{37,38}$ Indeed, early findings from one retrospective case series suggest that DRGS is an effective neurostimulation modality to improve painful symptoms in PDPN patients, ${ }^{16}$ and the results of another small retrospective case series suggest that DRGS may be an effective treatment option for painful hereditary and idiopathic axonal polyneuropathy. ${ }^{17}$

The aim of this prospective pilot study was to investigate the effect of DRGS on pain intensity in patients with painful intractable polyneuropathy. Secondary objectives were to assess the impact of DRGS on the patients' global impression of change (PGIC), the reduction of the pain extent, the presence of neuropathic pain, physical functioning, health related quality of life (HRQoL), and mood. 


\section{Materials and methods}

The ethics committee of the Canton Ticino, Switzerland approved this single-centre prospective pilot study. All subjects provided written informed consent prior to participation in the study. The study was registered at the Swiss Trial Register (SNCTP000001376) and at ClinicalTrials.gov (NCT02435004) and was performed at the Pain Management Center of the Neurocenter of Southern Switzerland.

\section{Participants}

Eligible subjects were at least 18 years or older and diagnosed with chronic, intractable painful polyneuropathy in the lower limbs, based on typical clinical signs and (stable) symptoms such as pinprick and thermal sensory loss, allodynia and hyperalgesia. ${ }^{10}$ All subjects were evaluated independently by the neurologist and were diagnosed with chronic large fibre polyneuropathy (LFN) (axonal, demyelinated, or mixed) through electroneuromyography (ENMG) and/or with small fibre neuropathy (SFN) confirmed by skin biopsy at the ankle showing a reduced intraepidermal nerve fibre (IENF) density. ${ }^{39,40}$ Furthermore, eligible patients had neuropathic pain for at least 3 months with an average daily and/or nighty pain intensity of $\geq 5$ on an 11-point Numeric Rating Scale (NRS) (ranging from 0 to 10, assessed by a pain diary of five days, three times daily and 1 time for the night) and had to present an incomplete analgesic effect and/or intolerable side effects with prior pharmacologic therapy, including at least antidepressants, anticonvulsants, or a combination of these. If painful polyneuropathy was also present in the upper limbs, patients were only eligible if the pain was at least predominantly present in the lower limbs. Patients were excluded if they suffered from pain in the limbs as a result of other origin than polyneuropathy (e.g. pain due to atherosclerotic lesions), if they were addicted to drugs or alcohol, and if they were known or suspected to be noncompliant, or incapable of cooperation (e.g. due to language problems, psychological disorders, dementia). Other exclusion criteria were coagulation disorders, known immune deficiency, infection at the site of skin incision, life expectancy of less than two years, pregnancy, and severe foraminal stenosis at the expected target level for DRGS lead implantation. All included subjects underwent screening for common etiologies of polyneuropathy, including levels of vitamin B1, B6, B12, folate, hemoglobin A1C, serum protein electrophoresis and immuno-fixation, thyroid-stimulating hormone (TSH), autoantibodies (antinuclear antibodies (ANA), extractable nuclear antigens (ENA) screen), HIV, hepatitis C virus (HCV) and Borrelia. 


\section{DRGS Implantation and Trial}

DRGS was delivered by the Proclaim ${ }^{\text {TM }}$ DRG Neurostimulator System (manufactured by St. Jude Medical, now Abbott, Sunnyvale, CA, USA). The participants were prepared for DRGS lead implantation according to our standard pain clinic's practice, including antibiotic prophylaxis, prone position and monitored anesthesia care. In each subject, between two and four quadripolar percutaneous DRGS leads were placed in the lateral epidural space at the level of the L5 and S1 dorsal root ganglion (DRG), depending on the dermatomal area of pain (Fig. 1A-1B). ${ }^{37,41,42}$ For the $L 5$ level the translaminar approach was used, while for the S1 level the needle was guided into the S1 foramen using the transforaminal approach, according to the technique descripted by Velsen et al. ${ }^{42}$ Intraoperative testing to determine the paresthesia coverage of the painful area was confirmed before lead fixation.

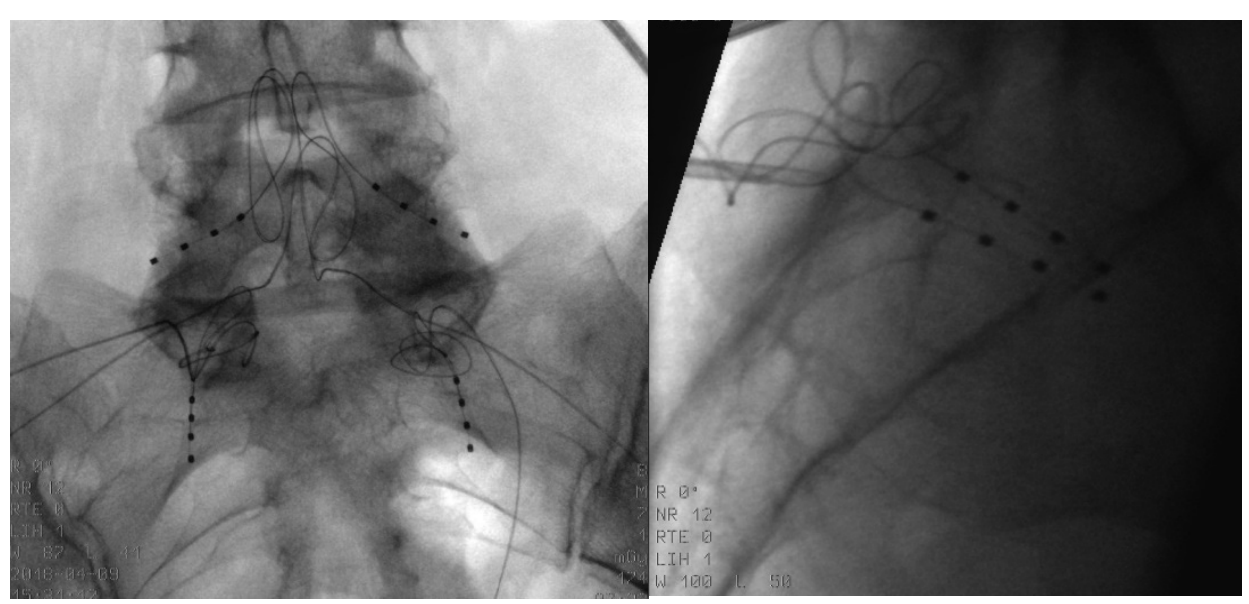

Figure 1. A) Anterior posterior fluoroscopic view of final position of bilateral DRGS leads on the L5 and S1 level. B) Lateral fluoroscopic view of final position of bilateral DRGS leads on the S1 level. DRGS, dorsal root ganglion stimulation.

For the trial phase of DRGS, the leads were connected via extension leads to an external pulse generator (EPG). The trial was defined to be successful if there was a reduction of $\geq 50 \%$ in daytime and/or night-time pain intensity during the trial period and if the patient was expressing a desire to be implanted with an implantable pulse generator (IPG). The average trial stimulation phase was $8(S D=2)$ days. In subjects with a successful trial, the extension leads were removed and the DRGS leads were connected to an IPG. The IPG was implanted subcutaneously in the upper buttock. Post implantation wound care and device programming proceeded according to standard practice. Device programming settings were adjusted for paresthesia to overlap maximally the painful areas. After programming, amplitude was reduced to remain subthreshold but therapeutic. 


\section{Primary outcome measure}

The primary outcome measure was pain intensity, which was evaluated using an 11-point NRS-based pain dairy as described above. ${ }^{43}$ The averages were calculated from the daily pain scores ( 3 times daily for 5 days) and nocturnal pain scores (once daily for 5 days). In addition, peak pain was calculated from the diaries. Clinical follow-ups of the primary outcome occurred at 1,3 , and 6 months post-implant. If trial stimulation had been unsuccessful, follow-up occurred only at 6 months.

\section{Secondary outcome measures}

Secondary outcomes were measured at 3 and 6 months. The PGIC of painful symptoms compared with baseline was assessed using the 7-point PGIC Likert scale. ${ }^{43}$ Additionally, pain extent was evaluated at baseline and follow up ( 3 and 6 months). Each patient completed a pain drawing using a sketching application installed on digital tablet (iPad Pro 12.9, Apple Computer, Cupertino, CA, USA). Patients were instructed to shade their current pain on two body charts (frontal and dorsal view of the body) using a stylus pen. Pain extent was estimated using a customized software previously validated, ${ }^{44,45}$ and was reported as the sum of all pixels coloured inside the two body charts and expressed as a percentage of the body charts area (dorsal body chart area, 145837 pixels; frontal body chart area, 14856 pixels). The pain frequency map is an illustration in which all the pain drawings are overlapped to highlight the most frequently reported locations of pain across the enrolled patients. Pain frequency maps and pain location analysis were computed as earlier described. ${ }^{46-49}$ Pain location analysis was obtained by dividing the body charts into 45 anatomical regions. ${ }^{50}$ The presence of pain in a body region was confirmed when the pain drawing involved at least $10 \%$ of the body region area or where the number of pixels was greater than 60. Moreover, histograms were used to quantify the number of patients that reported pain in a specific anatomical location.

Neuropathic pain assessment was performed with the Douleur Neuropathique 4 (DN4) questionnaire, which is considered a reliable tool with a high sensitivity and specificity, and positive predictive value. ${ }^{51}$ The DN4 consists of 7 items based on verbal pain description and 3 items related to clinical examination to be scored as 0 for "no" and 1 for "yes". A DN4 score of $\geq 4$ is considered as evidence of neuropathic pain. ${ }^{51}$

The interference of pain with functioning (general activity, mood, walking, work, relationships, sleep, and enjoyment of life) was measured with the Brief Pain Inventory (Short Form, BPISF). ${ }^{52}$ Zero is no interference and 10 is complete interference. Moreover, HRQoL was assessed using the EuroQol 5-dimention 5-level (EQ-5D-5L) health status measure. ${ }^{53}$ Mood was assessed with the Hospital Anxiety and Depression Scale (HADS), a self-report scale, originally developed to measure depression and anxiety among hospital outpatients clinics. ${ }^{54}$ 
The use of various types of analgesic medication was recorded at baseline and at follow up visits. Safety outcomes (frequency of adverse events [AEs] and severe adverse events [SAEs]) were recorded throughout the study.

\section{Statistical analysis}

Baseline characteristics of all included patients were summarized using mean and standard deviation and count and percentage. Pain intensity during the day and night and peak pain post-implantation were reported as mean and standard deviation, and as median and interquartile range (IQR), and compared to baseline values using the Wilcoxon signed-rank test, a non-parametric variant of the paired-samples t-test.

Scores reported on the PGIC were summarized as count and percentage. Pain extent was described as median and interquartile range. BPI pain interference, EQ-5D-5L health status, and depression and anxiety scores were compared with baseline values using the Wilcoxon signed-rank test. Stimulation parameters were described as mean, standard deviation and range, or as count and percentage. Analgesic use was stratified to type and reported as count and percentage. All analyses were performed using R version 3.5.1.

\section{Results}

Between September 2016 and January 2019, we screened 31 patients with painful polyneuropathy and 9 patients were included. Twenty-two patients were not eligible for inclusion: 5 patients had a pain intensity of $<5$ on the NRS; 3 patients suffered from pain in the limbs as a result of other origin, 12 patients declined to be implanted with a DRGS system, and one patient had a good analgesic effect of pharmacologic therapy (duloxetine in combination with tramadol). Baseline characteristics of all patients who were included are shown in Table 1. In Fig. 2 the pain distribution at baseline of the enrolled patients is shown. 

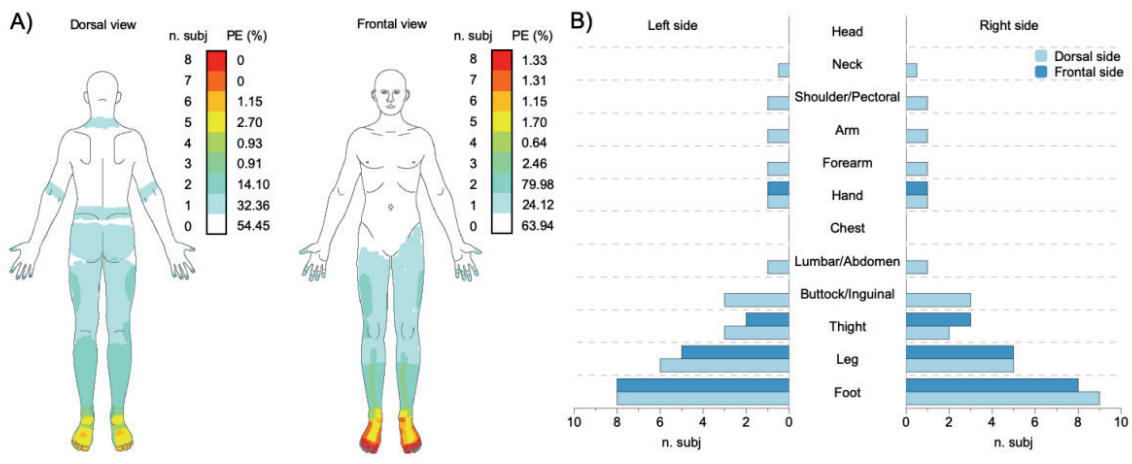

Figure 2. Pain distribution in the enrolled patients at the baseline. A) Pain frequency maps obtained by overlapping the pain drawings of all the patients enrolled in the study $(n=9)$. The colour scale indicates both the number patients that reported pain in the specific area and the extent of that area expressed in percentage of the total area of the body chart. Dark red represents the most frequently reported area of pain. B) Pain location analysis showing the number of patients reporting pain in a specific body region.

N. subj, number of subjects; PE, pain extent.

The median duration of painful polyneuropathy was 7 years. All patients that were included in this study were diagnosed with SFN. Five of these patients were also diagnosed with large fibre axonal polyneuropathy and 2 other patients with large fibre mixed polyneuropathy (Table 1). Eight out of 9 patients (88.9\%) had a successful trial phase, of which 7 received an IPG. In one of the patients with a successful trial phase, the DRGS leads were removed after the trial phase due to infection. The patient with the negative trial reported less than $50 \%$ pain relief during daytime and night-time and complained of symptoms of neurological deficit (see below). Table 1 reports the number and placement of leads in the subjects. Four subjects received bilateral implantation involving both L5 and S1 ganglion and five patients received bilateral implantation involving only the $\mathrm{L} 5$ ganglion. 
Table 1. Characteristics of all included patients

\begin{tabular}{|c|c|}
\hline & $\begin{array}{l}\text { All patients } \\
(\mathrm{N}=9)\end{array}$ \\
\hline Age & $63.2(8.7)$ \\
\hline \multicolumn{2}{|l|}{ Gender } \\
\hline Male & $6(66.7 \%)$ \\
\hline Female & $3(33.3 \%)$ \\
\hline $\mathrm{BMI}\left(\mathrm{kg} / \mathrm{m}^{2}\right)$ & $29.1(4.8)$ \\
\hline Smoking & $4(44.4 \%)$ \\
\hline Pain duration (years)* & 7 (1 to 20$)$ \\
\hline \multicolumn{2}{|l|}{ Aetiology } \\
\hline Diabetes mellitus type 2 & $3(28.6 \%)$ \\
\hline Idiopathic & $3(42.9 \%)$ \\
\hline Chemotherapy & $1(14.3 \%)$ \\
\hline CIDP & $2(14.3 \%)$ \\
\hline Douleur neuropathique score $>4$ & $8(88.9 \%)$ \\
\hline \multicolumn{2}{|l|}{ Polyneuropathy type } \\
\hline Small fiber neuropathy & $9(100 \%)$ \\
\hline Axonal & $5(71.4 \%)$ \\
\hline Demyelinated & $0(0.0 \%)$ \\
\hline Mixed & $2(28.6 \%)$ \\
\hline \multicolumn{2}{|l|}{ Number of leads implanted } \\
\hline 2 & $5(55.6 \%)$ \\
\hline 4 & $4(44.4 \%)$ \\
\hline \multicolumn{2}{|l|}{ Lead location } \\
\hline L5, left & $9(100 \%)$ \\
\hline L5, right & $9(100 \%)$ \\
\hline S1, left & $4(44.4 \%)$ \\
\hline S1, right & $4(44.4 \%)$ \\
\hline \multicolumn{2}{|l|}{ Preoperative medication use } \\
\hline Acetominophen & $2(22.2 \%)$ \\
\hline NSAID & $3(33.3 \%)$ \\
\hline Opioid, weak (tramadol, codeine) & $2(22.2 \%)$ \\
\hline Opioid, strong & $2(22.2 \%)$ \\
\hline Antidepressant & $1(11.1 \%)$ \\
\hline Muscle relaxant & $0(0.0 \%)$ \\
\hline Benzodiazepine & $1(11.1 \%)$ \\
\hline Antiepileptic & $5(55.6 \%)$ \\
\hline Systemic corticosteroid & $0(0.0 \%)$ \\
\hline Other (sleep medication, cannabis) & $0(0.0 \%)$ \\
\hline None & $0(0.0 \%)$ \\
\hline
\end{tabular}

Data are expressed as mean (standard deviation) or count (percentage), unless stated otherwise

BMI, body mass index; CIDP, chronic inflammatory demyelinating polyneuropathy; NSAID, nonsteroidal antiinflammatory drug.

*expressed as median (range) 


\section{Chapter 3}

\section{Primary outcome measure}

The remaining 7 patients completed their 6-month follow-up visit. On average, they showed a significant decrease of 4.5 points on the daytime pain NRS in the first week of the trial phase after leads implantation (Table 2). An average decrease of at least 3.4 points for daytime pain was found over the complete follow-up period. In addition to daytime pain, both night-time pain and peak pain during follow up decreased significantly. Fig. 3 shows average day and night pain scores over the 6 months follow-up. Six out of 7 IPG implanted patients (85.7\%) showed treatment success 1 month after treatment, defined as a minimum decrease of pain of $50 \%$ for day and night, and this remained stable during the 6 months follow up period.

Table 2. Pain scores of patients implanted with a device over the course of 6 months

\begin{tabular}{lllll}
\hline & $\begin{array}{l}\text { Mean } \\
\text { pain (SD) }\end{array}$ & $\begin{array}{l}\text { Mean difference with } \\
\text { Baseline }(95 \% \mathrm{CI})\end{array}$ & $\begin{array}{l}\text { Median pain } \\
(\text { IQR })\end{array}$ & $\begin{array}{l}\text { p-value for } \\
\text { difference }\end{array}$ \\
\hline $\begin{array}{llll}\text { Baseline } \\
\text { day }\end{array}$ & $\begin{array}{l}\text { N.0(2.1) } \\
\text { night }\end{array}$ & N.A. & $7.0(5.9-8.3)$ & N.A. \\
peak & $5.3(4.0)$ & N.A. & $5.4(2.1-8.9)$ & N.A. \\
Trial phase & $8.8(1.6)$ & N.A. & $9.0(9.0-10.0)$ & N.A. \\
1 week, day & $2.5(2.4)$ & $4.5(3.2-5.7)$ & $2.0(1.0-3.5)$ & 0.016 \\
1 week, night & $2.8(2.3)$ & $2.6(-0.1-5.2)$ & $2.0(1.7-3.4)$ & 0.106 \\
1 week, peak & $4.4(3.3)$ & $4.0(2.1-5.9)$ & $4.0(2.5-6.0)$ & 0.035 \\
Follow up & & & & \\
1 month, day & $2.7(2.0)$ & $4.3(2.6-5.9)$ & $2.3(1.5-3.7)$ & 0.016 \\
1 month, night & $2.6(2.8)$ & $2.7(0.9-4.5)$ & $2.2(0.0-4.7)$ & 0.036 \\
1 month, peak & $4.4(3.3)$ & $4.0(1.9-6.1)$ & $3.0(3.0-6.0)$ & 0.022 \\
3 months, day & $3.5(2.3)$ & $3.4(1.3-5.5)$ & $3.7(2.2-5.2)$ & 0.031 \\
3 months, night & $3.2(2.9)$ & $2.1(0.8-3.4)$ & $3.0(0.7-5.6)$ & 0.036 \\
3 months, peak & $4.6(2.5)$ & $3.9(1.5-6.2)$ & $5.0(3.5-6.5)$ & 0.020 \\
6 months, day & $3.1(2.1)$ & $3.9(1.4-6.3)$ & $3.0(1.6-4.9)$ & 0.031 \\
6 months, night & $2.4(3.0)$ & $2.9(0.5-5.4)$ & $1.0(0.0-4.2)$ & 0.036 \\
6 months, peak & $4.4(2.8)$ & $4.4(1.1-6.9)$ & $4.0(3.0-6.5)$ & 0.035 \\
\hline
\end{tabular}

$\mathrm{SD}$, standard deviation; $\mathrm{Cl}$, confidence interval; IQR, interquartile range; N.A., not applicable 


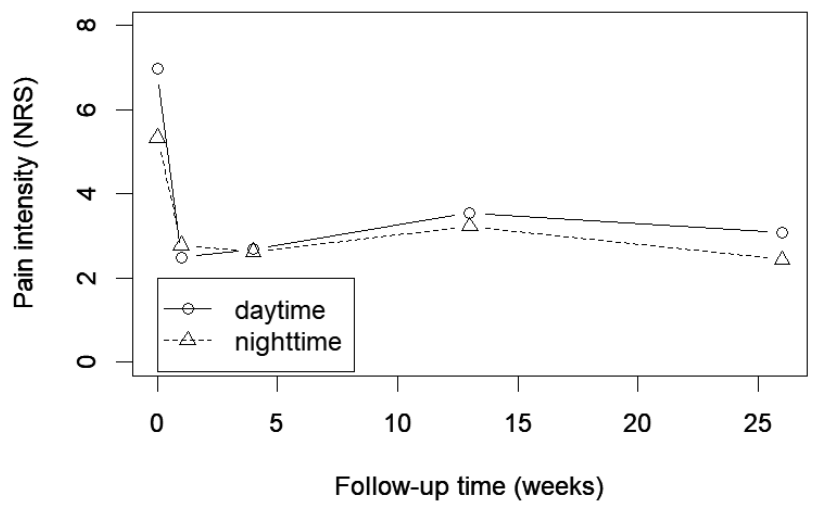

Figure 3. Mean NRS scores over 6 months follow-up, stratified by day and night. NRS, Numeric Rating Scale.

\section{Secondary outcome measures}

Fig. 4 shows the distribution of PGIC scores at 3 and 6 months. One of the IPG implanted patients reported worsening at 3 months, none at 6 months. At both time points, 6 out of $7(85.7 \%)$ reported improvement.

The median (IQR) pain extent was $7.7 \%(6.9)$ of the whole body at baseline and significantly reduced at 3 and 6 months to $2.4 \%$ (4.2) and 2.5\% (10.4), respectively. There was preoperative evidence of neuropathic pain according to the DN4 (score $\geq 4$ ) in 6 out of the 7 (85.7\%) IPG implanted patients. At 3 and at 6 months, 5 (71.4\%) of the 7 IPG implanted patients had a DN4 score $\geq 4$. Interference of pain with functioning (BPISF), $\mathrm{HRQ}$ oL, anxiety and depression scores are shown in Table 3. Both pain interference score decreased significantly after treatment.

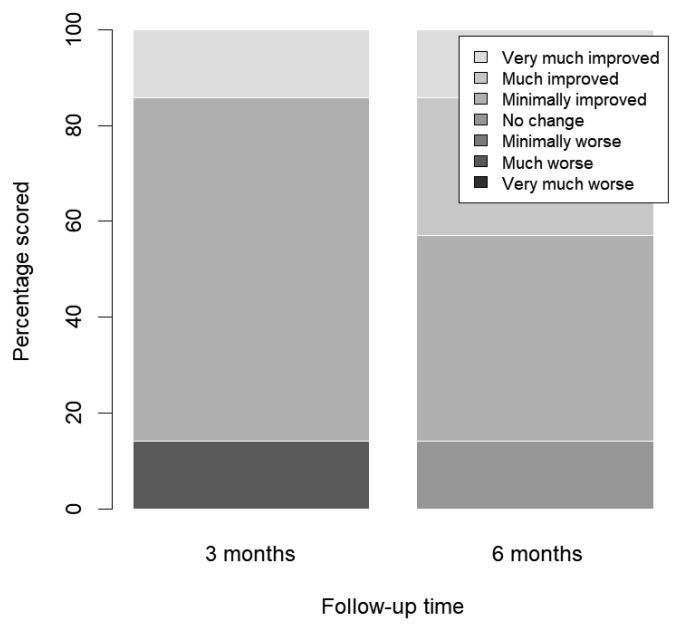

Figure 4. Distribution of Patient Global Impression of Change (PGIG) scores at 3 and 6 months. 
Chapter 3

Table 3. BPI, EQ-5D-5L, and HADS scores during follow-up compared to baseline

\begin{tabular}{llllll}
\hline & $\begin{array}{l}\text { Baseline } \\
\text { mean (SD) }\end{array}$ & $\begin{array}{l}3 \text { months } \\
\text { mean (SD) }\end{array}$ & $\begin{array}{l}3 \text { months } \\
\text { p-value }\end{array}$ & $\begin{array}{l}6 \text { months } \\
\text { mean (SD) }\end{array}$ & $\begin{array}{l}6 \text { months } \\
\text { p-value }\end{array}$ \\
\hline $\begin{array}{l}\text { BPI } \\
\text { Pain interference } \\
\text { EQ-5D-5L } \\
\text { Health-related QoL }\end{array}$ & $6.5(1.7)$ & $3.5(1.9)$ & 0.022 & $2.6(1.9)$ & 0.016 \\
$\begin{array}{l}\text { HADS } \\
\text { Anxiety } \\
\text { Depression }\end{array}$ & $69.3(28.3)$ & $60.7(23.9)$ & 0.518 & $59.3(18.6)$ & 0.620 \\
\hline
\end{tabular}

SD, standard deviation; BPI, Brief Pain Inventory; EQ-5D-5L, EuroQol 5-dimentoion 5-level, QoL, Quality of Life; HADS, Hospital Anxiety and Depression scale; N.A., not applicable.

$\mathrm{p}$-values are derived by comparing to baseline values

In Table 4 the programming settings used during the study are displayed. Most subjects had subthreshold stimulation settings. All patients were allowed to adjust stimulation amplitude and to switch the stimulation "off" and "on" as they wished. The majority of the patients used the stimulation continuously.

Table 4. Stimulation parameters

\begin{tabular}{|c|c|c|c|c|}
\hline & 3 months & & 6 months & \\
\hline & $L 5(n=7)$ & $\mathrm{S} 1(\mathrm{n}=2)$ & $\mathrm{L} 5(\mathrm{n}=7)$ & $\mathrm{S} 1(\mathrm{n}=2)$ \\
\hline \multicolumn{5}{|l|}{ Frequency $(\mathrm{Hz})$} \\
\hline Mean (SD) & $22.9(7.6)$ & $20(0)$ & $22.9(7.6)$ & $20(0)$ \\
\hline Min, $\max$ & 20,40 & 20,20 & 20,40 & 20,20 \\
\hline \multicolumn{5}{|l|}{ Pulse width ( $\mu s)$} \\
\hline Mean (SD) & $349.3(147.3)$ & $265.0(49.5)$ & $360.0(202.0)$ & $230(0)$ \\
\hline Min, max & $130.0,570.0$ & $230.0,300.0$ & 200,800 & 230,230 \\
\hline \multicolumn{5}{|l|}{ Amplitude ( $\mu \mathrm{A})$} \\
\hline Mean (SD) & 605.4 (379.9) & $181.2(8.8)$ & $598.2(473.3)$ & $187.5(0)$ \\
\hline Min, $\max$ & $137.5,1025.0$ & $175.0,187.5$ & $162.5,1462.5$ & $187.5,187.5$ \\
\hline \multicolumn{5}{|l|}{ Impedance } \\
\hline in the norm (\%) & $7 / 7$ (100\%) & $2 / 2(100 \%)$ & $7 / 7$ (100\%) & $2 / 2(100 \%)$ \\
\hline
\end{tabular}

Preoperatively, all subjects were taking analgesics (Table 1 and Table 5). Most subjects were treating pain with anticonvulsants (pregabaline, carbamazepine) (Table 1). Four patients $(44.4 \%)$ were taking opioids, of whom half took strong opioids (fentanyl and oxycodone). In the IPG implanted patients there was a tendency in reduced analgesic intake (Table 5), but we refrained from statistical testing due to the limited number of patients per type of analgesic. 
Table 5 Analgesics during follow-up compared to baseline of implanted patients

\begin{tabular}{llll}
\hline & Preoperative & 3 months & 6 months \\
\hline $\begin{array}{l}\text { Acetaminophen } \\
\text { paracetamol) }\end{array}$ & $2(28.6 \%)$ & $1(14.3 \%)$ & $1(14.3 \%)$ \\
NSAID & $3(42.9 \%)$ & $1(14.3 \%)$ & $1(14.3 \%)$ \\
$\begin{array}{l}\text { Opioid, weak (tramadol, } \\
\text { codeine) }\end{array}$ & $2(28.6 \%)$ & $2(28.6 \%)$ & $2(28.6 \%)$ \\
Opioid, strong & $2(28.6 \%)$ & $1(14.3 \%)$ & $1(14.3 \%)$ \\
Antidepressant & $1(14.3 \%)$ & $1(14.3 \%)$ & $1(14.3 \%)$ \\
Muscle relaxant & $0(0.0 \%)$ & $0(0.0 \%)$ & $0(0.0 \%)$ \\
Benzodiazepine & $1(14.3 \%)$ & $1(14.3 \%)$ & $1(14.3 \%)$ \\
Antiepileptic & $3(42.9 \%)$ & $2(28.6 \%)$ & $2(28.6 \%)$ \\
Systemic corticosteroid & $0(0.0 \%)$ & $0(0.0 \%)$ & $0(0.0 \%)$ \\
Other (sleep medication, & $0(0.0 \%)$ & $0(0.0 \%)$ & $0(0.0 \%)$ \\
cannabis) & & $0(0.0 \%)$ & $0(0.0 \%)$ \\
None & $0(0.0 \%)$ & &
\end{tabular}

NSAID, nonsteroidal anti-inflammatory drug.

Two AEs occurred in 2 subjects. In one patient wound dehiscence at the site of needle insertion was noted at the day of IPG implant. The lead was notable, but there were no signs of infection. During the intervention of the IPG implant, the wound was revised and sutured again. The subject was dismissed on the same day and the wound healed well. In another subject, who was implanted with two leads at the bilateral L5 DRG, one lead was erroneously cut by the surgeon during the IPG implant. This AE was resolved through lead repositioning.

Two SAE's occurred in 2 subjects. One subject, which had a negative trial phase, suffered from loss of sensitivity in the $L 5$ dermatome and deficit of dorsiflexion of the right foot during the trail phase. Possible relation with the device (radicular lead irritation) was suspected and the leads were removed. In the following months the neurologic symptoms resolved. As in this patient the trial was negative for pain relief, leads were not re-implanted. The other SAE, which occurred in a patient suffering from insulin dependent DM, was a subcutaneous infection at the site of the connections between the DRGS leads and the extension leads at the end of the trial phase. This SAE was interpreted as definitely related the implant procedure. The patient responded well to intravenous antibiotic treatment and the removal of the DRGS leads. It was decided to not re-implant the leads in this high-risk patient. 


\section{Discussion}

Our study is the first prospective pilot study that suggests that DRGS results in a clinical significant pain reduction for at least six months follow up in patients with refractory painful polyneuropathy in the lower limbs. DRGS reduced daytime pain, night-time pain and peak pain. Trial stimulation with DRGS was successful in 8 out of 9 patients (89\%) with intractable painful polyneuropathy, which is higher than most previously published SCS studies in the treatment of PDPN. ${ }^{18,19,28,30,31}$ In 7 of these patients a definite IPG was implanted. Six out of the 7 IPG implanted patients (85.7\%) showed stable treatment outcome during the follow up period of 6 months and an improvement on the PGIC.

In our study, the baseline mean pain interference (impact of pain on functioning) was 6.5, which is high in comparison to earlier studies in PDPN patients. ${ }^{55,56}$ However mean pain interference decreased significantly to 2.6 with DRGS treatment. Additionally, mood improved, although not significantly.

DRGS reduced pain extent significantly at 3 and 6 months as compared to baseline. Indeed, during this study, the standard pain assessment tools/procedures have been completed by providing patients with a digital tablet including body charts. All patients were able to provide a detailed topographic measurement of their pain, highlighting its somatic location together with the extension. From a clinical perspective, the observed shrinking of the pain should be considered as a meaningful finding especially when considering that previous investigations confirmed that pain extent is associated with disability and psychological factors. ${ }^{57-59}$ As clearly confirmed by the pain frequency maps of this study, pain in polyneuropathy is mostly present in the feet. DRGS has been shown to cover difficult-to-reach areas like the feet better than $\mathrm{SCS},{ }^{37,41}$ and therefore we hypothesize that DRGS is more effective when compared to SCS in painful polyneuropathy patients. This hypothesis is supported by the fact that the results of a randomized controlled trial confirmed a higher rate of treatment success of DRGS than SCS in CRPS. ${ }^{60}$

An important aspect of our prospective study as compared to published clinical studies on effectiveness of SCS in PDPN, ${ }^{14,18,27-34}$ is the fact that the aetiology of polyneuropathy in our study was heterogeneous and not confined to PDPN. In the present study, the significant pain relieving effect in the subsample of the 2 PDPN patients was similar as compared to the 5 patients with polyneuropathy of other etiologies (idiopathic, CIDP, chemotherapy-induced peripheral neuropathy). This suggest that DRGS has the ability to not only successfully manage pain in PDPN, but also in other etiologies of painful polyneuropathy. This is even more important as the prevalence of other conditions leading to painful polyneuropathies is rising. ${ }^{61-63}$ Additionally, several case reports and case series have already suggested that SCS and DRGS have the ability to successful manage pain in polyneuropathy of other etiologies than PDPN. . $2,21,64^{2}$

As DRGS implantation is an invasive and expensive treatment, it should only be offered to well selected patients and as a last resort treatment. Therefore, we used strict 
inclusion criteria like a pain intensity of $\geq 5$ on an NRS, responding incompletely to pharmacologic therapy. Moreover, although the clinical history and physical examination are often sufficient to make the diagnosis of a SFN and a LFN, additional objective diagnostic tests help to confirm the diagnosis. ${ }^{65}$ Our study was the first prospective neurostimulation study in polyneuropathy patients that restricted inclusion to patients that were objectively diagnosed with SFN and/or LFN using diagnostic tests like nerve conduction studies, EMG and skin biopsy. Consequently, it was confirmed that the patients were suffering from "definitive neuropathic pain" according to the grading system of the International Association for the Study of Pain (IASP) Special Interest Group on Neuropathic Pain (NeuPSIG). ${ }^{66}$

In the current study, HRQoL did not improve significantly. However, this may be caused by a lack of statistical power for this outcome in our study. Pain is known to be a primary indicator for worsening HRQoL and diminished overall wellbeing in polyneuropathy patients. ${ }^{67-69}$ A study regarding PDPN patients has previously shown that PDPN patients have a significantly worse HRQoL compared with DM patients without pain. ${ }^{70}$ Moreover, HRQoL is inversely associated with neuropathic pain severity and pain duration, ${ }^{68,71}$ and the aetiology of polyneuropathy does not influence levels of neuropathic pain-related compromise of HRQoL. ${ }^{67}$ Furthermore, it is known that neuropathic pain reduction, as a result of treatment, is related to improvement in HRQoL. ${ }^{72,73}$ Although in the current study HRQoL did not improve, studies with a larger number of subjects are probably necessary to determine the effects of DRGS on HRQoL.

Most IPG implanted patients of our study were able to reduce their analgesic intake. As effectiveness of pharmacological treatment in painful polyneuropathy patients is often minor and frequently accompanied by unacceptable side effects, ${ }^{12,13}$ these patients are more likely to have medication incompliance, like inappropriate polypharmacy and misuse of prescribed medications. ${ }^{67}$ Many patients require pain treatment with more than one analgesic, which increases the risk of additional adverse events and incorrect use of analgesics. Furthermore, concomitant medication use is high in these patients. For instance, a patient survey has shown that $43 \%$ of PDPN patients received prescription medications for sleep disturbance, anxiety and/or depression. ${ }^{74}$ The fact that most IPG implanted patients of our study were able to reduce their analgesic intake, suggests that DRGS has the advantage of minimizing the use of analgesics in painful polyneuropathy patients, thereby also reducing the risk of drug interactions.

As expected for an invasive procedure, DRGS implantation is not without risks. Two SAE's related to the DRGS implant occurred in the current study, and both led to the removal of the trial leads. The patient that suffered from an infection was diabetic. His trial period had a duration of 11 days, a little shorter than the standard duration of 14 days applied in most SCS/DRGS-studies. However, it could be argued that in patients susceptible to infections, like DM, a maximum trial period of 7 days should be applied, to lessen the risk for infection. The SAE characterized by neurological deficit in the second patient was transitory and eventually resolved. 
The present study has some limitations. First, it was conducted with a relatively small number of patients, although the number of patients was large enough to demonstrate clinically relevant and statistically significant effects on pain reduction over a 6-months period. Studies with a larger number of subjects are necessary to determine the effects of DRGS on HRQoL. Additionally, due to the heterogeneity of etiologies of polyneuropathy, our pilot study does not allow to draw definite conclusions about DRGS for painful polyneuropathy of singular etiologies. However this study suggest that DRGS is significantly effective for the treatment of painful polyneuropathy in general, independently from the underlying cause.

In conclusion, the results of this study suggest that DRGS reduces significantly both pain intensity and PGIC in patients with refractory painful polyneuropathy in the lower extremities. Additionally, clinically improvements have been appreciated for pain extent, impact of pain on functioning, mood, and analgesics intake. A successful application of digital pain drawings has been reported. This low cost and user-friendly tools should be considered, in clinical practice as well as in research, to optimize and monitor DRGS treatments. Larger-scale (randomized controlled) clinical trials are needed to prove the efficacy of DRGS in intractable pain associated with polyneuropathy. 


\section{References}

1. Hanewinckel R, van Oijen M, Ikram MA, et al. The epidemiology and risk factors of chronic polyneuropathy. Eur J Epidemiol. 2016;31:5-20.

2. Callaghan BC, Price RS, Feldman EL. Distal symmetric polyneuropathy a review. JAMA. 2015;314:21722181.

3. Beghi E, Monticelli ML. Chronic symmetric symptomatic polyneuropathy in the elderly: a field screening investigation in two italian regions. I. Prevalence and general characteristics of the sample. Neurology. 1995;45:1832-1836.

4. Visser NA, Notermans NC, Linssen RS, et al. Incidence of polyneuropathy in Utrecht, the Netherlands. Neurology. 2015;84:259-264.

5. Rudolph T, Farbu E. Hospital-referred polyneuropathies-causes, prevalences, clinical- and neurophysiological findings. Eur J Neurol. 2007;14:603-608.

6. Navis A, Jiao J, George MC, et al. Comorbid pain syndromes in HIV-associated peripheral neuropathy. Pain Med. 2017;19:1445-1450.

7. Ventzel $L$, Jensen $A B$, Jensen AR, et al. Chemotherapy-induced pain and neuropathy: A prospective study in patients treated with adjuvant oxaliplatin or docetaxel. Pain. 2016;157:560-568.

8. Bennett DL, Woods CG. Painful and painless channelopathies. Lancet Neurol. 2014;13:587-599.

9. Abbott CA, Malik RA, van Ross ER, et al. Prevalence and characteristics of painful diabetic neuropathy in a large community-based diabetic population in the U.K. Diabetes Care. 2011;34:2220-2224.

10. Watson JC, Dyck PJB. Peripheral neuropathy: A practical approach to diagnosis and symptom management. Mayo Clin Proc. 2015;90:940-951.

11. Breivik H, Collett B, Ventafridda V, et al. Survey of chronic pain in Europe: Prevalence, impact on daily life, and treatment. Eur J Pain. 2006;10:287-333.

12. Bril V, England J, Franklin GM, et al. Evidence-based guideline: treatment of painful diabetic neuropathy. PM\&R. 2011;3:345-352.

13. Sindrup SH, Jensen TS. Pharmacologic treatment of pain in polyneuropathy. Neurology. 2000;55:915-920.

14. van Beek M, Slangen R, Schaper NC, et al. Sustained treatment effect of spinal cord stimulation in painful diabetic peripheral neuropathy: 24-month follow-up of a prospective two-center randomized controlled trial. Diabetes Care. 2015;38:e132-134.

15. van Beek M, Geurts JW, Slangen R, et al. Severity of neuropathy is associated with long-term spinal cord stimulation outcome in painful diabetic peripheral neuropathy: Five-year follow-up of a prospective twocenter clinical trial. Diabetes Care. 2018;41:32-38.

16. Eldabe S, Espinet A, Wahlstedt A, et al. Retrospective case series on the treatment of painful diabetic peripheral neuropathy with dorsal root ganglion stimulation. Neuromodulation. 2018;21:787-792.

17. Ho KWD, Rempe $\mathrm{T}$, Jerath $\mathrm{N}$, et al. Dorsal root ganglion stimulation as a potentially effective treatment for painful hereditary and idiopathic axonal polyneuropathy: A retrospective case series. Neuromodulation. 2019;23:234-238.

18. Slangen R, Schaper NC, Faber CG, et al. Spinal cord stimulation and pain relief in painful diabetic peripheral neuropathy: A prospective two-center randomized controlled trial. Diabetes Care. 2014;37:3016-3024.

19. De Vos CC, Meier K, Zaalberg PB, et al. Spinal cord stimulation in patients with painful diabetic neuropathy: A multicentre randomized clinical trial. Pain. 2014;155:2426-2431.

20. Abd-Elsayed A, Schiavoni N, Sachdeva H. Efficacy of spinal cord stimulators in treating peripheral neuropathy: A case series. J Clin Anesth. 2016;28:74-77.

21. Knezevic NN, Candido KD, Rana S, et al. The use of spinal cord neuromodulation in the management of HIV-related polyneuropathy. Clin J Sport Med. 2016;18:E643-E650.

22. Kemler MA, Barendse GA, van Kleef $M$, et al. Spinal cord stimulation in patients with chronic reflex sympathetic dystrophy. N Engl J Med. 2000;343:618-624. 


\section{Chapter 3}

23. Kumar K, Taylor RS, Jacques L, et al. Spinal cord stimulation versus conventional medical management for neuropathic pain: A multicentre randomised controlled trial in patients with failed back surgery syndrome. Pain. 2007;132:179-188.

24. Tsigaridas N, Naka K, Tsapogas $P$, et al. Spinal cord stimulation in refractory angina. A systematic review of randomized controlled trials. Acta Cardiol. 2015;70:233-243.

25. Eldabe S, Thomson S, Duarte R, et al. The effectiveness and cost-effectiveness of spinal cord stimulation for refractory angina (RASCAL study): A pilot randomized controlled trial. Neuromodulation. 2016;19:6070 .

26. Ubbink DT, Vermeulen H. Spinal cord stimulation for non-reconstructable chronic critical leg ischaemia. Cochrane Database Syst Rev. 2013;2013:CD004001.

27. Kumar K, Toth C, Nath RK. Spinal cord stimulation for chronic pain in peripheral neuropathy. Surg Neurol. 1996;46:363-369.

28. Tesfaye S, Watt J, Benbow SJ, et al. Electrical spinal-cord stimulation for painful diabetic peripheral neuropathy. Lancet. 1996;348:1698-1701.

29. Daousi C, Benbow SJ, MacFarlane IA. Electrical spinal cord stimulation in the long-term treatment of chronic painful diabetic neuropathy. Diabet Med. 2005;22:393-398.

30. de Vos CC, Rajan V, Steenbergen W, et al. Effect and safety of spinal cord stimulation for treatment of chronic pain caused by diabetic neuropathy. J Diabetes Complicat. 2009;23:40-45.

31. Pluijms WA, Slangen R, Bakkers $M$, et al. Pain relief and quality-of-life improvement after spinal cord stimulation in painful diabetic polyneuropathy: A pilot study. Br J Anaesth. 2012;109:623-629.

32. Slangen R, Pluijms WA, Faber CG, et al. Sustained effect of spinal cord stimulation on pain and quality of life in painful diabetic peripheral neuropathy. Br J Anaesth. 2013;111:1030-1031.

33. de Vos C, Meier K, Zaalberg P, et al. Spinal cord stimulation in patients with painful diabetic neuropathy: a multicentre randomized clinical trial. Pain. 2014;155:2426-2431.

34. Geurts JW, Joosten EA, van Kleef M. Current status and future perspectives of spinal cord stimulation in treatment of chronic pain. Pain. 2017;158:771-774

35. Verrills $P$, Sinclair C, Barnard A. A review of spinal cord stimulation systems for chronic pain. J Pain Res. 2016;9:481-492.

36. Aló KM, Holsheimer J. New trends in neuromodulation for the management of neuropathic pain. Neurosurgery. 2002;50;690-703.

37. Liem L, Russo M, Huygen FJPM, et al. A multicenter, prospective trial to assess the safety and performance of the spinal modulation dorsal root ganglion neurostimulator system in the treatment of chronic pain. Neuromodulation. 2013;16:471-482.

38. Schu S, Gulve A, Eldabe S, et al. Spinal cord stimulation of the dorsal root ganglion for groin pain-A retrospective review. Pain Pract. 2015;15:293-299.

39. Devigili G, Tugnoli V, Penza P, et al. The diagnostic criteria for small fibre neuropathy: From symptoms to neuropathology. Brain. 2008;131:19112-1925

40. Boruchow SA, Gibbons $\mathrm{CH}$. Utility of skin biopsy in management of small fiber neuropathy. Muscle Nerve. 2013;48:877-882

41. Liem L, Russo M, Huygen FJPM, et al. One-year outcomes of spinal cord stimulation of the dorsal root ganglion in the treatment of chronic neuropathic pain. Neuromodulation. 2015;18:41-49.

42. van Velsen V, van Helmond N, Chapman KB. Creating a strain relief loop during S1 transforaminal lead placement for dorsal root ganglion stimulation for foot pain: A technical note. Pain Pract. 2018;18:539543.

43. Dworkin RH, Turk DC, Wyrwich KW, et al. Interpreting the clinical importance of treatment outcomes in chronic pain clinical trials: IMMPACT recommendations. J Pain. 2008;9:105-121.

44. Leoni $D$, Falla $D$, Heitz $C$, et al. Test-retest reliability in reporting the pain induced by a pain provocation test: further validation of a novel approach for pain drawing acquisition and analysis. Pain Pract, 2017;17:176-184.

45. Barbero $M$, Moresi $F$, Leoni $D$, et al. Test-retest reliability of pain extent and pain location using a novel method for pain drawing analysis. Eur J Pain. 2015;19:1129-1138. 
46. Willett MJ, Siebertz M, Petzke F, et al. The extent of pain is associated with signs of central sensitization in patients with hip osteoarthritis. Pain Pract. 2019;20:277-288.

47. Barbero M, Fernández-de-las-Peñas $C$, Palacios-Ceña $M$, et al. Pain extent is associated with pain intensity but not with widespread pressure or thermal pain sensitivity in women with fibromyalgia syndrome. Clin Rheumatol. 2017;36:1427-1432.

48. Fernandez-de-Las-Peñas C, Falla D, Palacios-Ceña M, et al. Perceived pain extent is not associated with physical, psychological, or psychophysical outcomes in women with carpal tunnel syndrome. Pain Med (United States). 2019;20:1185-1192.

49. Cruder C, Falla D, Mangili F, et al. Profiling the Location and Extent of Musicians' Pain Using Digital Pain Drawings. Pain Pract. 2018;18:53-66.

50. Margolis RB, Tait RC, Krause SJ. A rating system for use with patient pain drawings. Pain. 1986;24:57-65.

51. Bouhassira D, Attal N, Fermanian J, et al. Development and validation of the Neuropathic Pain Symptom Inventory. Pain. 2004;108:248-257.

52. Tan G, Jensen MP, Thornby Jl, et al. Validation of the brief pain inventory for chronic nonmalignant pain. J Pain. 2004;5:133-137.

53. Herdman M, Gudex C, Lloyd A, et al. Development and preliminary testing of the new five-level version of EQ-5D (EQ-5D-5L). Qual Life Res. 2011;20:1727-1736.

54. Iani L, Lauriola M, Costantini M. A confirmatory bifactor analysis of the hospital anxiety and depression scale in an Italian community sample. Health Qual Life Outcomes. 2014;9:521-526.

55. Tölle T, Xu X, Sadosky AB. Painful diabetic neuropathy: A cross-sectional survey of health state impairment and treatment patterns. J Diabetes Complications. 2006;20:26-33.

56. Alleman $\mathrm{CJ}$, Westerhout KY, Hensen M, et al. Humanistic and economic burden of painful diabetic peripheral neuropathy in Europe: A review of the literature. Diabetes Res Clin Pract. 2015;109:215-225.

57. Ris I, Barbero M, Falla D, et al. Pain extent is more strongly associated with disability, psychological factors, and neck muscle function in people with non-traumatic versus traumatic chronic neck pain: A cross sectional study. Eur J Phys Rehabil Med. 2019;55:71-78.

58. Palacios-Ceña M, Barbero M, Falla D, et al. Pain extent is associated with the emotional and physical burdens of chronic tension-type headache, but not with depression or anxiety. Pain Med. 2017;18:20332039.

59. Falla D, Peolsson A, Peterson G, et al. Perceived pain extent is associated with disability, depression and self-efficacy in individuals with whiplash-associated disorders. Eur J Pain. 2016;20:1490-1501.

60. Deer TR, Levy RM, Kramer J, et al. Dorsal root ganglion stimulation yielded higher treatment success rate for complex regional pain syndrome and causalgia at 3 and 12 months: A randomized comparative trial. Pain. 2017;158:669-681.

61. De La Monte SM, Kril JJ. Human alcohol-related neuropathology. Acta Neuropathol. 2014;127:71-91.

62. Kaku M SD. HIV neuropathy. Curr Opin HIV AIDS. 2014;9:521-526.

63. Colvin LA. Chemotherapy-induced peripheral neuropathy: where are we now? Pain. 2019;160:Suppl 1:S1S10.

64. Groenen PS, van Helmond N, Chapman KB. Chemotherapy-induced peripheral neuropathy treated with dorsal root ganglion stimulation. Pain Med. 2018;20:857-859.

65. Hovaguimian A, Gibbons CH. Diagnosis and treatment of pain in small-fiber neuropathy. Curr Pain Headache Rep. 2011;15:193-200.

66. Finnerup NB, Haroutounian S, Kamerman P, et al. Neuropathic pain: An updated grading system for research and clinical practice. Pain. 2016;157:1599-1606.

67. Poliakov I, Toth C. The impact of pain in patients with polyneuropathy. Eur J Pain. 2011;15:1015-1022.

68. Gore M, Brandenburg NA, Dukes E, et al. Pain severity in diabetic peripheral neuropathy is associated with patient functioning, symptom levels of anxiety and depression, and sleep. I Pain Symptom Manage. 2005;30:374-385.

69. van Acker K, Bouhassira D, de Bacquer D, et al. Prevalence and impact on quality of life of peripheral neuropathy with or without neuropathic pain in type 1 and type 2 diabetic patients attending hospital outpatients clinics. Diabetes Metab. 2009;35:206-213. 


\section{Chapter 3}

70. Davies M, Brophy S, Williams R, Taylor A. The prevalence, severity, and impact of painful diabetic peripheral neuropathy in type 2 diabetes. Diabetes Care. 2006;29:1518-1522.

71. Geelen CC, Smeets RJEM, Schmitz S, et al. Anxiety affects disability and quality of life in patients with painful diabetic neuropathy. Eur J Pain. 2017;21:1632-1641.

72. Deshpande MA, Holden RR, Gilron I. The impact of therapy on quality of life and mood in neuropathic pain: What is the effect of pain reduction? Anesth Analg. 2006;102:1473-1479.

73. Attal N, Cruccu G, Baron R, et al. EFNS guidelines on the pharmacological treatment of neuropathic pain: 2010 revision. Eur J Neurol. 2010;17:111-e88.

74. Rodríguez MJ, Díaz S, Vera-Llonch M, et al. Cost-effectiveness analysis of pregabalin versus gabapentin in the management of neuropathic pain due to diabetic polyneuropathy or post-herpetic neuralgia. Curr Med Res Opin. 2007;23:2585-2596. 


\section{Chapter 4}

\section{Effectiveness of Dorsal Root Ganglion Stimulation and Dorsal Column Spinal Cord Stimulation in a Model of Experimental Painful Diabetic Polyneuropathy}

Eva Koetsier, MD, ${ }^{1}$ Glenn Franken, $\mathrm{MSc}^{2,3}$ Jacques Debets, ${ }^{4}$ Sander M.J. van Kuijk $\mathrm{PhD},{ }^{5}$ Roberto S.G.M. Perez ${ }^{6}$, PhD, Bengt Linderoth, PhD, MD, ${ }^{7}$ Elbert A. Joosten, PhD, ${ }^{2,3}$ Paolo Maino, MD ${ }^{1}$

${ }^{1}$ Pain Management Center, Neurocenter of Southern Switzerland, Regional Hospital of Lugano, Via Capelli, 6962, Lugano, Switzerland

${ }^{2}$ Department of Anesthesiology and Pain Management, Maastricht University Medical Center ${ }^{+}$, Maastricht, the Netherlands

${ }^{3}$ Department of Translational Neuroscience, School of Mental Health and Neuroscience (MHeNS), University of Maastricht, the Netherlands

${ }^{4}$ Muroidean Facility, School of Cardiovascular Diseases (CARIM), Maastricht, the Netherlands

${ }^{5}$ Department of Clinical Epidemiology and Medical Technology Assessment, Maastricht University Medical Center ${ }^{+}$, Maastricht, the Netherlands.

${ }^{6}$ Department of Anesthesiology and Pain Management, VU University Medical Center, Amsterdam, the Netherlands

${ }^{7}$ Department of Clinical Neuroscience, Karolinska Institutet, SE-171 77 Stockholm, Sweden

CNS Neuroscience \& Therapeutics. 2019;25:367-37 


\begin{abstract}
Aims: Conventional Dorsal Root Ganglion Stimulation (DRGS) is known to achieve better pain-paresthesia overlap of difficult-to-reach areas like the feet compared to dorsal column Spinal Cord Stimulation (SCS). As in Painful Diabetic polyneuropathy (PDPN) pain is mostly present in the feet, we hypothesized that DRGS is more effective in relieving pain in PDPN when compared to SCS.
\end{abstract}

Methods: Diabetes was induced in female Sprague-Dawley rats with an intraperitoneal injection of $65 \mathrm{mg} / \mathrm{kg}$ of streptozotocin (STZ) ( $\mathrm{n}=48$ ). Rats with a significant decrease in mechanical paw withdrawal response to von Frey filaments 4 weeks after injection were implanted with DRGS electrodes $(n=18)$. Rats were assigned to DRGS $(n=11)$ or sham$\operatorname{DRGS}(n=7)$. Mechanical paw Withdrawal Thresholds (WT, measured in grams) in response to DRGS $(50 \mathrm{~Hz}, 0.18 \pm 0.05 \mathrm{~mA})$ were assessed with von Frey testing. The results of the experiments on these animals were compared to the results of a previous study using exactly the same model on PDPN animals selected for SCS $(n=8)(40-50 \mathrm{~Hz}, 0.19 \pm$ $0.01 \mathrm{~mA}$ ) and Sham-SCS ( $\mathrm{n}=3)$.

Results: In the SCS group, the $\log _{10}(10.000 \times 50 \%$ WT $)$ increased from 4.910 to 5.211 at $\mathrm{t}=15 \mathrm{~min}(p<0.05)$, and 5.264 at $\mathrm{t}=30 \mathrm{~min}(\mathrm{p}=0.11)$. In the DRGS group, the $\log _{10}(10.000$ $x$ 50\% WT) increased from 4.376 to 4.809 at $t=15 \mathrm{~min}(p<0.01)$ and 5.042 at $t=30 \mathrm{~min}$ $(p<0.01)$. Both DRGS and SCS induced a similar and complete reversal of mechanical hypersensitivity. After cessation of stimulation $(t=60)$ the return of the $\log _{10}(10.000 \mathrm{x}$ $50 \% \mathrm{WT}$ ) response was significantly faster with DRGS than that of SCS $(p<0.05)$.

Conclusions: We conclude that conventional DRGS is as effective as SCS in reduction of PDPN associated mechanical hypersensitivity in STZ-induced diabetic rats. The wash-in effect of DRGS and SCS was similar, but DRGS showed a faster wash-out course. Longterm efficacy should be studied in future animal research. 


\section{Introduction}

Diabetic Polyneuropathy (DPN) is a chronic, symmetrical, length-dependent sensorimotor polyneuropathy, and is present in up to $50 \%$ of patients with diabetes mellitus. ${ }^{1}$ One third of these DPN patients suffers from Painful Diabetic Polyneuropathy (PDPN), ${ }^{2}$ which starts in the toes and spreads into the feet, legs and hands. ${ }^{3}$ As PDPN can be debilitating and a severe handicap to the patient and since effectiveness of pharmacological drugs is limited, there is an urgent need for other treatment options. Conventional Spinal Cord Stimulation of the dorsal columns (hereafter named SCS) has been shown to be such a treatment option, which as well can be supplementary to pharmacological therapy. SCS has shown to be effective on the short- and long-term in PDPN when pharmacological therapies have failed. ${ }^{4-12}$ However, conventional SCS often provides incomplete pain relief (50\% pain reduction or even less), ${ }^{10,11}$ which is restricted to $60 \%$ of PDPN patients and leaves $40 \%$ of the patients as non-responders. In view of these limitations a recently introduced and very promising option for treatment of PDPN might be conventional Dorsal Root Ganglion Stimulation (DRGS). DRGS is known to achieve better pain-paresthesia overlap of difficult-to-reach areas like the feet. ${ }^{13,14}$ The results of a recently published retrospective case series suggest that DRGS improves painful symptoms in PDPN patients. ${ }^{15}$ As in PDPN pain is mostly present in the feet, ${ }^{16}$ we hypothesized that DRGS is more effective in pain relief in PDPN when compared to SCS.

In order to address this hypothesis, we implemented DRGS in an already operational and meticulously tested PDPN animal model and investigated the effectiveness of both DRGS and SCS. SCS and its resulting pain relief in Streptozotocin (STZ) induced PDPN animals has recently been described and here it was shown that SCS normalizes STZinduced mechanical hypersensitivity. ${ }^{17}$ SCS resulted in "a clinically relevant reduction" of mechanical hypersensitivity in 70\% of PDPN animals. DRGS in animals with peripheral nerve damage and chronic neuropathic pain has been described recently. ${ }^{18}$ DRGS did not cause any dorsal root ganglion (DRG) tissue damage as verified by histological examination and with the use of DRGS parameters closely replicating those in clinical use a significant reduction of mechanical hypersensitivity in chronic neuropathic animals was noted. ${ }^{18}$ In order to investigate the underlying pain relieving effect of DRGS in PDPN animals, we compared the pain relieving effect of DRGS versus SCS in PDPN associated mechanical hypersensitivity in STZ-induced diabetic rats. 


\section{Methods}

This study aimed to investigate the behavioral effect (mechanical hypersensitivity as measured by paw withdrawal to von Frey filaments) of one single stimulation paradigm in PDPN: a 30 minutes (min) conventional DRGS (hereafter named DRGS) being compared to a 30 min SCS in rats with PDPN. ${ }^{16}$ This manuscript adheres to the applicable ARRIVE guidelines.

\section{Animals}

The experiments for this study were performed using 18 female Sprague-Dawley rats, which were 8 weeks of age at the start of the experiment (170-230 g). Eleven rats were selected for active DRGS and seven rats were selected for Sham-DRGS. Animals were either housed in pairs before DRGS device implantation, and individually after DRGS device implantation in transparent plastic cages with free access to food and water, in a 12-h light dark cycle. The experiments were approved by the Animal Research Committee of the Maastricht University Medical Centre (DEC-protocol and approval (DEC 2013-079)). The results of the experiments on these animals were compared to the results of a previous study investigating effect of conventional SCS but otherwise performed in an identical manner as this study ( $n=8$ for SCS and $n=3$ for Sham-SCS). ${ }^{17}$

\section{Induction of diabetes mellitus}

DM was induced with a single intraperitoneal injection of $65 \mathrm{mg} / \mathrm{kg}$ streptozotocin (STZ, Sigma-Aldrich, Schnelldorf, Germany) in 48 animals. Before STZ injection, rats were weighed and fasted overnight. STZ was freshly dissolved in sterile $\mathrm{NaCl} 0.9 \%$ to a solution of $65 \mathrm{mg} / \mathrm{mL}$. Four days after STZ injection, blood glucose level was determined in blood derived from the saphenous vein of the leg using a standard blood glucose meter (AccuChek Aviva ${ }^{\circledR}$, Roche Diagnostics $\mathrm{GmbH}$, Mannheim, Germany). Rats with a glucose level of $\geq 15 \mathrm{mmol} / \mathrm{L}$ were considered diabetic, ${ }^{19}$ and were included in the study.

\section{Behavioural testing}

Pain behavior was assessed by testing mechanical hypersensitivity based on the hind limb paw withdrawal response to von Frey filaments. Before the start of behavioural testing, rats were placed in a transparent box on an elevated mesh floor and were given 15 min to acclimate to the surroundings. Mechanical hypersensitivity was assessed according to the 'up-down method', ${ }^{20}$ as previously described. ${ }^{17,21}$ A cut-off value of $28.84 \mathrm{~g}$ was defined. Thereafter, the registered $50 \%$ paw withdrawal thresholds (WT), measured in grams, were multiplied by 10.000 and then logarithmically transformed to conform with Weber's law ${ }^{22}$ and obtain a linear scale. The average of the mechanical hypersensitivity of both paws in the SCS animals of the previous study ${ }^{17}$ was compared to the average of 
the mechanical hypersensitivity of the ipsilateral (stimulated) paw in the DRGS animals, as we did not expect ipsilateral DRGS to have any effects on the contralateral hind paw.

\section{Inclusion of animals}

Animals were tested for mechanical hypersensitivity using von Frey hind limb withdrawal testing at baseline (before STZ injection), and once a week for 4 weeks following STZ injection, for the purpose of selecting animals that develop PDPN. Only animals showing mechanical hypersensitivity at 4 weeks post-injection were treated with either SCS or with DRGS, whereas animals showing no mechanical hypersensitivity were excluded from the study. The presence of mechanical hypersensitivity was presumed if the $\log _{10}(10.000$ x 50\% WT) decreased by 0.2 units compared with pre-STZ baseline. ${ }^{17,21}$

\section{Preparation electrode for DRG stimulation}

The DRGS leads were manufactured from two platinum-iridium wires of different gauges, where the larger diameter wire (0.010in) contained the smaller center wire (0.005in) (PlasticsOne, Roanoke, VA, see Fig 1; see Pan et al. ${ }^{18}$ ), that were secured together at one end in a plastic connection hub. To prepare the electrode for DRGS implantation, the insulation from the terminal portion of the larger wire was removed and the terminal portion of the wire was folded back upon itself to produce an atraumatic tip (Fig. 1). The insulation was similarly removed from the tip of the smaller gauge wire, which was wrapped helically around the insulated portion of the central wire. This design produced an axially symmetric devise that was insensitive to rotational movement. We added a few spots of dental cement to stabilize the structure of the electrode and to give a place for an encircling suture to grab when securing the inserted electrode. The lead was tested with an Ohmmeter to confirm that there was no contact between the two electrode poles and that there was suitable low resistance to the terminal contacts (i.e. no break in the wires) (Fig.1). 


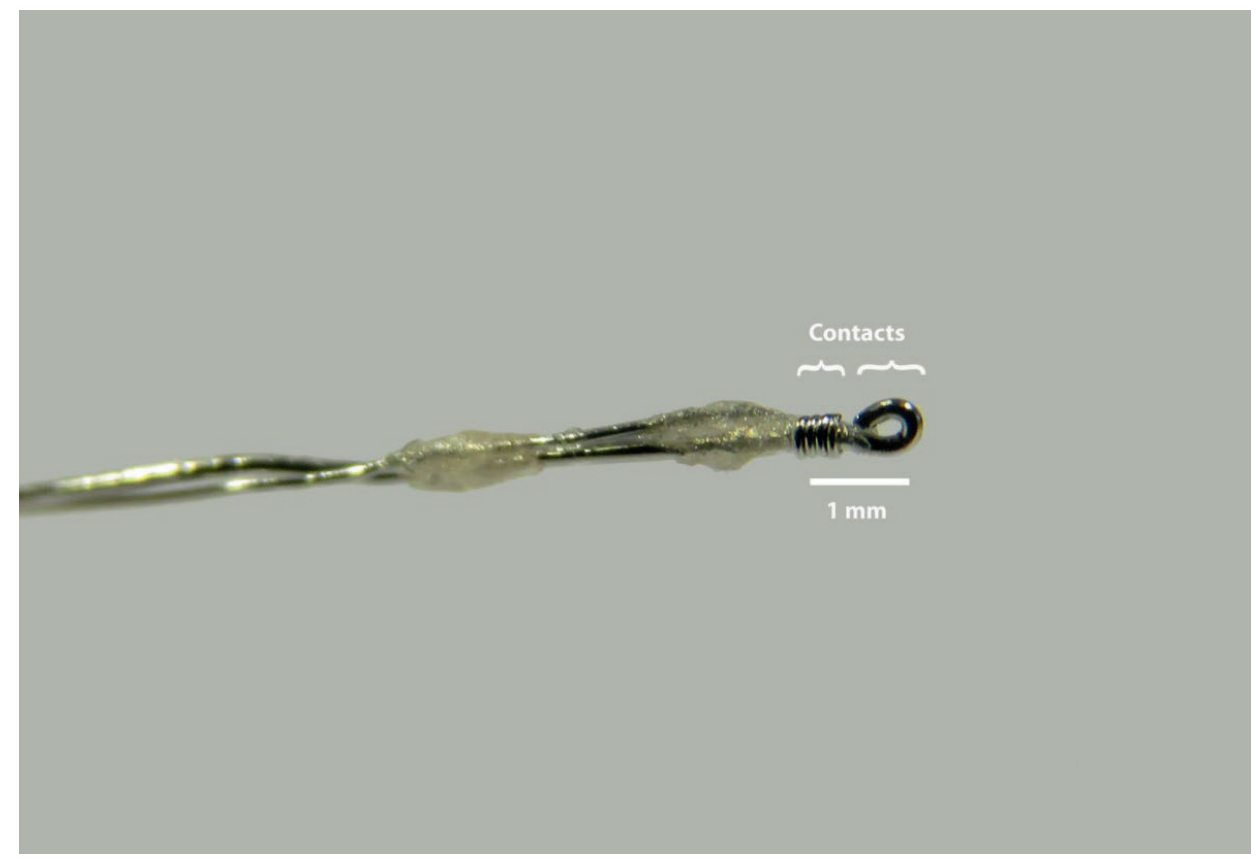

Figure 1 Bipolar electrode for dorsal root ganglion stimulation

Bipolar DRGS electrode. The insulation from the terminal portion of the larger wire was removed and the terminal portion of the wire was folded back upon itself to produce an atraumatic tip. The insulation was similarly removed from the end of the smaller gauge wire, which was then wrapped helically around the insulated portion of the central wire (see Pan et al. 2016). ${ }^{18}$ Both the anode and cathode were implanted at the L5 DRG.

\section{Implantation of DRGS device}

A bipolar DRGS electrode was implanted unilaterally at the L5 DRG (adapted from Pan et al.). ${ }^{18}$ In short: for implantation of the DRGS device the lateral aspect of the intervertebral foramen was exposed via a paravertebral incision under general anaesthesia. Subsequently, the foramen was gently opened by probing with a small, blunt nerve hook to provide a passage for the electrode to enter the foramen on the dorsolateral aspect of the DRG. Both the anode and cathode were implanted at the L5 DRG. The electrode was secured into the transverse process caudal to the foramen using a stainless steel ligature and a small screw (diameter 0,86 mm, length 3,2 $\mathrm{mm}$ ).

\section{Implantation of dorsal column SCS device}

The implantation of the dorsal column SCS device has been previously published and the description is included here to document the difference between the techniques. For the implantation of the SCS device, performed under general anaesthesia, a small laminectomy was made at level T13 and the cathode was inserted in the epidural space 
in caudal direction. Subsequently, the wire was secured to vertebra T12 with tissue adhesive (Histoacryl ${ }^{\circledR}$, B Braun Medical BV, Oss, the Netherlands) to prevent migration of the electrode. The anode was placed subcutaneously in the left flank. ${ }^{16}$

\section{SCS and DRGS}

After implantation of a given device (DRGS or SCS) in the separate groups, the cables were tunnelled subcutaneously through the neck of the animals, sutured to muscle and skin, and the connectors also attached to the skin. The wound was closed in layers. After implantation of the device, the rats were allowed to recover for 2 days before the start of SCS at day 3 following implantation.

The animals were stimulated in one session for $30 \mathrm{~min}$ at 3 days post-implantation. After the connector was attached to the wire of the pulse generator (for SCS: Grass S 88 stimulator fitted with a Grass SIU-5 stimulus isolation unit and a Grass constant current unit (Astro Med, Grass, Warwick, RI, USA); for DRGS: A-M systems MultiStim Model 3800, fitted with an A-M systems 3820 stimulus isolator (A-M systems, Sequim, WA, USA)), the motor threshold (MT) was determined at the following settings for both SCS and DRGS: frequency of $2 \mathrm{~Hz}$ and pulse width of $0.2 \mathrm{~ms}$. MT was defined as the current inducing contractions of the lower trunk or hind limb(s). Stimulation was applied for 30 min with an intensity of $66.7 \%$ of the MT, a pulse width of $0.2 \mathrm{~ms}$, and with a frequency of $40-50$ $\mathrm{Hz}$ in the SCS animals (amplitude: $0.19 \pm 0.01 \mathrm{~mA}$ ) and $50 \mathrm{~Hz}$ in the DRGS animals $(0.18 \pm$ $0.05 \mathrm{~mA}$ ). For the sham animals the amplitude was set at zero.

The animals were tested for mechanical hypersensitivity immediately before stimulation ( $=4$ weeks after STZ injection), 15 ( $\mathrm{t}=15 \mathrm{~min}$ ) and $30 \mathrm{~min}$ ( $\mathrm{t}=30 \mathrm{~min}$ ) during stimulation, and $30 \mathrm{~min}$ after stimulation ( $\mathrm{t}=60 \mathrm{~min}$ ). The measurements at 15 and 30 min during stimulation were performed with the pulse generator switched on. The effect of stimulation was assessed as follows: Firstly, the mean $\log _{10}(10.000 \times 50 \%$ WT) after the start of stimulation (at 15 and $30 \mathrm{~min}$ ) was compared with the mean $\log _{10}(10.000 \mathrm{x}$ $50 \%$ WT) before stimulation onset. Secondly, the mean $\log _{10}(10.000 \times 50 \%$ WT) after the start of stimulation (at 15 and $30 \mathrm{~min}$ ) was compared with the mean Pre-STZ $\log _{10}(10.000$ $x 50 \%$ WT). Thirdly, the effect size was assessed by calculation of the difference between the pre-STZ mean $\log _{10}(10.000 \times 50 \%$ WT $)$ and the mean $\log _{10}(10.000 \times 50 \%$ WT $)$ at 15 and 30 , and $60 \mathrm{~min}$ after the start of stimulation. Lastly, the percentage of responders to stimulation treatment was also calculated. A responder to stimulation was defined as an animal with an increase of the $\log _{10}(10.000 \times 50 \% \mathrm{WT}) \geq 0,2$ during stimulation at 15 and 30 min after the start of stimulation treatment. ${ }^{17}$

\section{Statistical analysis}

Data are presented as means and standard error of mean (SEM). Within-group analysis of changes of mechanical WT over time were analysed using the Wilcoxon signed-rank test. Between-group comparisons of mechanical WT and group characteristics were 
Chapter 4

analysed using the Mann-Whitney U-test and Kruskall-Wallis test. Statistical significance was defined as $p<0.05$.

\section{Results}

\section{Description of cohorts of animals}

Starting from 48 animals, which were injected with STZ, 43 developed DM (blood glucose $\geq 15 \mathrm{mmol} / \mathrm{L} ; 90 \%)$. PDPN developed in 22 animals ( $\log _{10}(10.000 \times 50 \%$ WT) decreased $\geq 0.2$ ); $51 \%$ ), and those animals were implanted with a DRGS device. Four of these 22 animals were excluded from the study, due to connector breakage $(n=2)$ and due to having a too high MT ( $>1 \mathrm{~mA})(\mathrm{n}=2)$. The remaining 18 animals were included for this study and divided into two groups: DRGS $(n=11)$ and sham-DRGS $(n=7)$. For SCS 11 PDPN animals were used as a historical cohort and as described by Pluijms et al. ${ }^{17}$ From these 11 rats 8 were stimulated with SCS (mid-frequency SCS group; $50 \mathrm{~Hz}$ ) and 3 control rats underwent sham-SCS. No significant differences were found between groups with respect to glucose levels [SCS $25.4 \pm 1.5 \mathrm{mmol} / \mathrm{L}$, Sham-SCS $23.4 \pm 4.0 \mathrm{mmol} / \mathrm{L}$, DRGS 27.8 $\pm 1.2 \mathrm{mmol} / \mathrm{L}$, and Sham-DRGS $29.2 \pm 0.9 \mathrm{mmol} / \mathrm{L}(p=0.12)]$.

\section{Development of mechanical hypersensitivity}

In the SCS group, the $\log _{10}(10.000 \times 50 \%$ WT) decreased from 5.412 before STZ injection to 4.910 before start of stimulation $(p<0.01)$. In the DRGS group, the $\log _{10}(10.000 \times 50 \%$ WT) decreased from 5.059 before STZ injection to 4.376 before start of stimulation ( $p<$ 0.01). In the Sham- SCS group, the $\log _{10}(10.000 \times 50 \%$ WT) showed a trend towards a decrease from 5.404 at Pre-STZ baseline to 4.918 before the start of the Sham-SCS therapy $(p=0.25)$. The $\log _{10}(10.000 \times 50 \%$ WT $)$ in the Sham-DRGS group significantly decreased from 5.041 before STZ injection to 4.416 before the start of Sham-DRGS therapy $(p<0.05)$.

\section{Effect of SCS / DRGS on mechanical hypersensitivity}

In the SCS group, stimulation resulted in an increase of the $\log _{10}(10.000 \times 50 \%$ WT $)$ from 4.910 before SCS to 5.211 at $\mathrm{t}=15 \mathrm{~min}(p<0.05)$, and a non-significant trend towards an increase to 5.264 at $t=30 \mathrm{~min}$ (relative to the pre-stimulation baseline $p=0.11$ ). No differences were observed between the $15 \mathrm{~min}$ and $30 \mathrm{~min}$ time point with SCS $(p=0.69)$.After SCS was stopped, the $\log _{10}(10.000 \times 50 \% \mathrm{WT})$ returned to pre-SCS values at $\mathrm{t}=60 \mathrm{~min}(5.093 \mathrm{~g} ; \mathrm{p}=0.20)$. In the DRGS group, the $\log _{10}(10.000 \times 50 \% \mathrm{WT})$ increased from 4.376 before the start of DRGS treatment to 4.809 at $t=15 \min (p<0.01)$ and increased to 5.042 at $t=30 \mathrm{~min}$ relative to the pre-stimulation baseline $(p<0.01)$. A significant increase was observed between the $15 \mathrm{~min}$ and $30 \mathrm{~min}$ time point with DRGS 
$(p<0.05)$. After cessation of DRGS therapy, the $\log _{10}(10.000 \times 50 \%$ WT $)$ returned to preDRGS values at $\mathrm{t}=60 \mathrm{~min}(4.451 ; p=0.90)$. After cessation of the stimulation ( $\mathrm{t}=60 \mathrm{~min}$ ) the wash-out effect, the $\log _{10}(10.000 \times 50 \%$ WT) with DRGS was significantly lower than that of SCS $(p<0.05)$ (data presented as \% of pre-STZ; Fig. 2).

\section{Mechanical hypersensitivity: SCS vs DRGS}

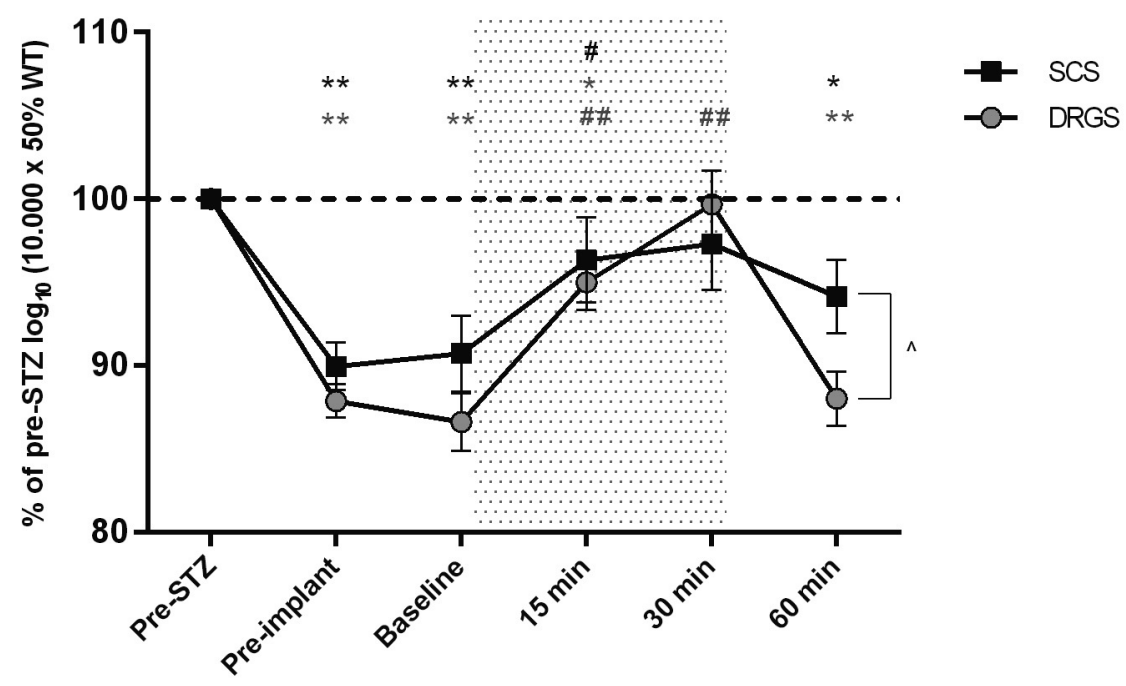

Figure 2 Effect of SCS ( $n=8)$ and DRGS ( $n=11)$ on mechanical hypersensitivity

Data are presented as mean \% of pre-STZ $\log 10(10.000 \times 50 \%$ WT $) \pm$ SEM. Data are compared to pre-STZ values and pre-stimulation baseline values. The stippled area denotes the stimulation period. (also in Fig 3). ${ }^{*} p<0.05$, ${ }^{* *} p<0.01$ compared to pre-STZ values; \#p<0.05, \#\#p<0.01 compared to pre-SCS baseline; $p<0.05$.

SCS, Spinal cord stimulation; DRGS, Dorsal Root Ganglion Stimulation; STZ, streptozotocin; SEM, Standard Error of Mean; min, minutes. 


\section{Effect of Sham-SCS / Sham-DRGS on mechanical hypersensitivity}

Sham therapy - as expected - did not result in a significant increase of the $\log _{10}(10.000$ $x 50 \%$ WT) in the Sham-SCS group at $t=15 \min (4.778, p=0.75)$, and at $t=30 \min (4.796$, $p=0.99)$, and neither in the Sham-DRGS group at $t=15 \min (4.354, p=0.56)$, and at $t=30$ $\min (4.382, p=0.69)$. No significant differences in terms of $\log _{10}(10.000 \times 50 \%$ WT) were found between the Sham-DRGS and Sham-SCS group (data presented as \% of pre-STZ; Fig. 3).

\section{Mechanical hypersensitivity: Sham-SCS vs Sham-DRGS}

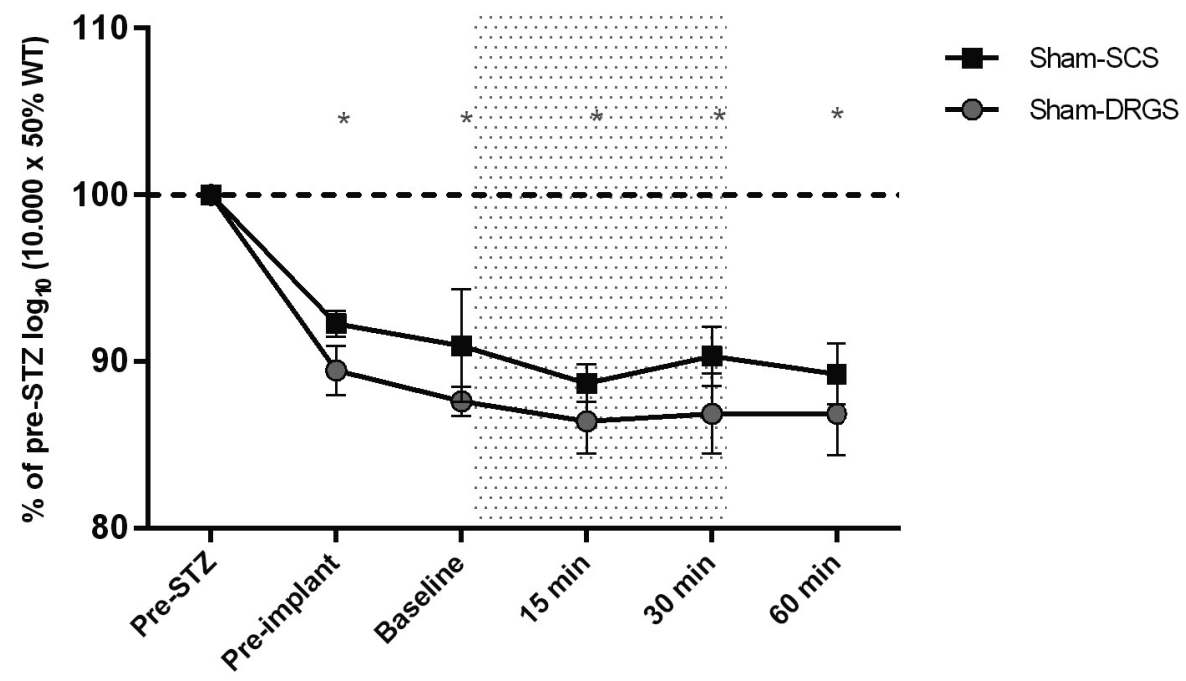

Figure 3 Effect of Sham SCS ( $n=3)$ and Sham DRGS ( $n=7)$ on mechanical hypersensitivity Data are presented as mean $\%$ of pre-STZ $\log _{10}(10.000 \times 50 \%$ WT $) \pm$ SEM. Data are compared to pre-STZ values and pre-stimulation baseline values. ${ }^{*} \mathrm{p}<0.05$ compared to pre-STZ values.

SCS, Spinal cord stimulation; DRGS, Dorsal Root Ganglion Stimulation; STZ, streptozotocin; SEM, Standard Error of Mean; min, minutes.

\section{Percentage responders}

In the SCS cohort the percentage of responders to stimulation was 75\% (six out of eight) at $\mathrm{t}=15$ and $30 \mathrm{~min}$, whereas in the DRGS cohort, the percentage of responders was $73 \%$ (eight out of eleven) at $\mathrm{t}=15 \mathrm{~min}$ and $91 \%$ (ten out of eleven) at $\mathrm{t}=30 \mathrm{~min}$. Sham-SCS did not result in a response on mechanical hypersensitivity both at $\mathrm{t}=15 \mathrm{~min}(0 / 3)$ and at $\mathrm{t}=30$ $\min (0 / 3)$ whereas Sham-DRGS resulted in 1 responder only at $t=30 \mathrm{~min}(1 / 7,14 \%)$ (Table 1). 
Table 1 Percentage responders to stimulation

\begin{tabular}{lll}
\hline Group & $T=15 \mathrm{~min}$ & $\mathrm{~T}=30 \mathrm{~min}$ \\
\hline SCS & $6 / 8(75 \%)$ & $6 / 8(75 \%)$ \\
DRGS & $8 / 11(73 \%)$ & $10 / 11(91 \%)$ \\
Sham-SCS & $0 / 3(0 \%)$ & $0 / 3(0 \%)$ \\
Sham-DRGS & $0 / 7(0 \%)$ & $1 / 7(14 \%)$ \\
\hline
\end{tabular}

A responder to stimulation is defined as an animal with an increase of the $\log _{10}(10.000 \times 50 \%$ WT $) \geq 0,2$ during stimulation.

SCS, Spinal Cord Stimulation; DRGS, Dorsal Root Ganglion Stimulation; WT, Withdrawal Threshold; min, minutes.

\section{Effect size of SCS and DRGS: comparison with pre-STZ values}

In the SCS group, the $\log _{10}(10.000 \times 50 \%$ WT $)$ returned to pre-STZ values after $\mathrm{t}=15 \mathrm{~min}(p$ $=0.31$ ) and $\mathrm{t}=30 \mathrm{~min}(p=0.69)$. In the DRGS group, the $\log _{10}(10.000 \times 50 \% \mathrm{WT})$ returned to pre-STZ values after $\mathrm{t}=30 \mathrm{~min}(p>0.99)$ (data presented as \% of pre-STZ; Fig. 2 ).

\section{Intergroup comparison of effect size of SCS and DRGS on mechanical}

hypersensitivity

Intergroup comparison of the effect size showed no differences between the SCS cohort and the DRGS cohort at $15 \mathrm{~min}(p=0.30)$ and at $30 \mathrm{~min}(p=0.13)$, and $60 \min (p=0.59)$ (Figs 2-4). (data presented as \% of pre-STZ (Figs 2-4).

\section{Effect size}

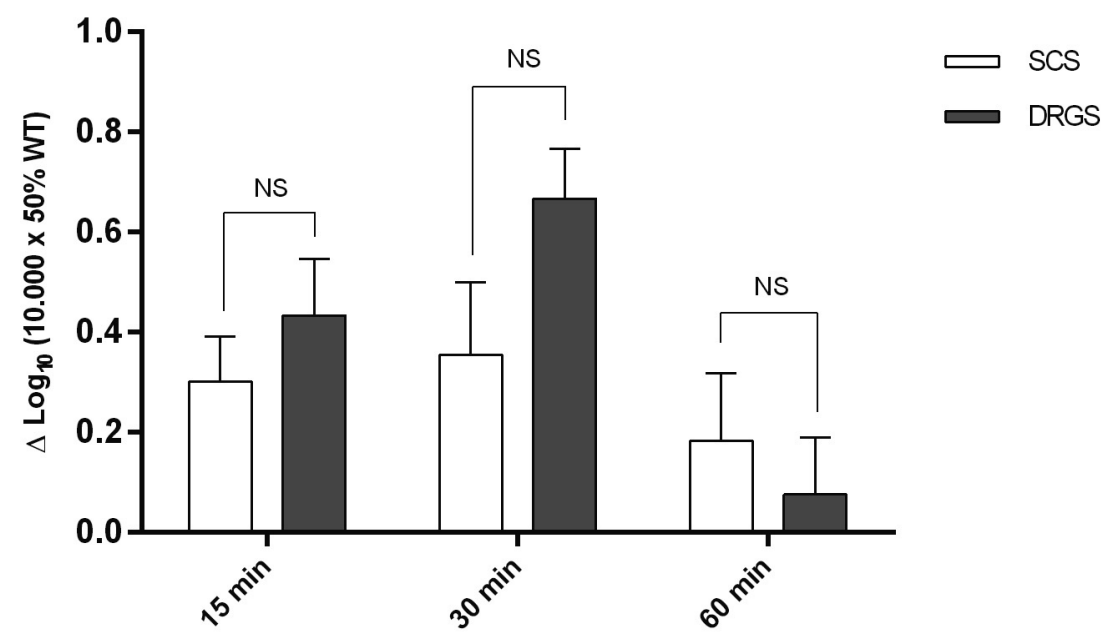

Figure 4 Reversal of mechanical hypersensitivity after 15, 30 and 60 min of SCS or DRGS Data of SCS and DRGS at 15, 30 and 60 min minus their respective pre-stimulation baseline at each time point. Data are presented as mean $\Delta \log _{10}(10.000 \times 50 \%$ WT $) \pm$ SEM. $N=8$ for SCS and $n=11$ for DRGS.

SCS, Spinal cord stimulation; DRGS, Dorsal Root Ganglion Stimulation; SEM, Standard Error of Mean, NS, Not Significant; min, minutes. 


\section{Discussion}

This is the first study that analyzes the behavioral pain relieving effect of DRGS in PDPN animals. From our results, we conclude that DRGS is as effective as SCS for PDPN associated mechanical hypersensitivity in STZ-induced diabetic rats. Both DRGS and SCS induced a complete reversal of mechanical hypersensitivity and a return to pre-STZ values. The complete reversal was obtained already at $15 \mathrm{~min}$ in the SCS group versus 30 min in the DRGS group. The percentage of responders was similar in both treatment groups. The wash-in effect of DRGS and SCS was also similar, with the exception that the therapeutic effect of SCS remained stable between $15 \mathrm{~min}$ and $30 \mathrm{~min}$, while in the DRGS group the therapeutic effect increased between these time points. DRGS showed a faster wash-out course $30 \mathrm{~min}$ after cessation of stimulation in comparison to SCS.

The pathophysiology of diabetic neuropathy includes metabolic changes, which affect nerve fibers and conduction velocity as well as blood microvessel alterations. ${ }^{1}$ Painful stimuli are transmitted by peripheral nerves along small non myelinated (C-type) and thinly myelinated $\left(A_{\delta}\right)$ fibres. These fibres are involved in diabetic polyneuropathy, as along with large myelinated fibres $\left(A_{\alpha}\right.$ and $\left.A_{\beta}\right) \cdot{ }^{23}$ It is conceived that SCS activates fastconducting thick $A_{\beta}$ fibers, ${ }^{24}$ and that electrical stimulation in the dorsal column results in antidromic activation of the descending collateral branches. According to the GateControl Theory, this stimulation of $A_{\beta}$ fibers results in modulation of the incoming $C$ - or $\mathrm{A}_{\delta}$ - mediated nociceptive signal. ${ }^{25}$ Animal studies have shown that SCS provides a suppressive action on dorsal horn neuronal hyperexcitability. ${ }^{26}$

The DRG mediates nociception from the peripheral nerves to the central nervous system, ${ }^{27}$ and is an appealing site for neurostimulation as it represents the sensory gateway to the spinal cord, containing sensory neuron somata for all sensory modalities and fiber types. At the DRG it is theoretically possible to modulate not only the nonnociceptive $A_{\beta}$ fibers, but also the nociceptive $A_{\delta}$ - and $C$-type fibers ${ }^{25}$ Furthermore, the DRG is of great importance in the development and maintenance of chronic pain, as it exhibits pathophysiologic changes during chronic pain states, like altered electrophysiological membrane properties, changes in the expression of integral membrane proteins, and altered gene expression. ${ }^{28-30}$ Elevated excitability of sensory neurons in the DRG contributes to the pathogenesis of chronic pain that follows peripheral nerve injury, ${ }^{31}$ and treatment at this site may interact with the pathogenic processes. It is known that DRGS reduces the generation of action potentials by the sensory neurons during membrane depolarization, which suggest that DRGS provides its analgesic effect via reducing sensory neuron excitability, both on spontaneous activation within the DRG and exerting an inhibitory influence on small diameter fiber activity passage at the T-junction. ${ }^{25}$

Conventional SCS (pulse width 0.2-0.5 ms; amplitude of 3.6 to $8.5 \mathrm{~mA}$; frequency of $40-80 \mathrm{~Hz})^{32,33}$ has been shown to be effective in patients having a variety of neuropathic pain conditions, including PDPN. ${ }^{10,12,34}$ DRGS is, with proper lead placement, effective for 
pain localized to the back, groin, legs, and feet. ${ }^{13,35-37}$ While SCS is often unable to cover difficult-to-reach areas like the feet and the groin without generating extensive paresthesias or motor side effects, ${ }^{13}$ conventional DRGS (settings: pulse width 0.2-0.4 ms; amplitude of 800 to $900 \mu \mathrm{A}$; frequency of $20-70 \mathrm{~Hz})^{13,35}$ has been shown to be able to cover these difficult-to-reach areas, without generating large unwanted areas of paresthesia. DRGS offers several other potential benefits over SCS systems like lack of positional and movement effects on stimulation and reduced migration rate, because of better lead stability. ${ }^{1,13,27}$ Additionally, as the anatomical location of the DRG offers a closer proximity to the electrodes compared to the spinal cord and its dorsal columns, reduced power is required. ${ }^{1,13}$

As PDPN pain is mostly located in the feet, we expected DRGS to be more effective for PDPN pain relief then SCS. Nevertheless, our results showed that effectiveness for pain relief with DRGS and SCS is similar in PDPN animals, with use of conventional stimulation settings. It needs to be stressed that these experiments were based on shortterm stimulation paradigms. Therefore, long-term efficacy should be studied in future animal research to analyse and detect possible differences between the effect of DRGS and SCS in PDPN. In this study, the therapeutic pain relieving effect of SCS remained stable between $15 \mathrm{~min}$ and $30 \mathrm{~min}$, while in the DRGS group the therapeutic effect increased between these time points. This suggests that a longer stimulation time would probably benefit mostly for DRGS, as treatment with SCS reached maximal pain relief effects after 30 minutes (or possibly even earlier). A possible advantage of DRGS as compared to SCS in PDPN could well appear only in a long-term stimulation study. ${ }^{15}$

Furthermore, as the concept underlying DRGS differs from the SCS (DRGS likely to modulate also nociceptive $C$ and $A_{\delta}$ fibers, whereas SCS only stimulates $A_{\beta}$ fibers) it is of the utmost importance to test various stimulation settings in an experimental model to optimize the pain relieving effect. Novel advances in neurostimulation frequencies have emerged, like High Frequency SCS (HF SCS, with frequencies up to $10 \mathrm{kHz}$ ) and Burst SCS (frequency $40 \mathrm{~Hz}$ provided in bursts of five pulses, with an internal frequency of $500 \mathrm{~Hz}{ }^{25}$ Van Beek et. al evaluated the effect of SCS frequency $(5-500 \mathrm{~Hz})$ on mechanical hypersensitivity in the chronic phase of experimental PDPN. ${ }^{21} \mathrm{~A}$ higher frequency (500 $\mathrm{Hz}$ ) SCS resulted in a delayed effect on the pain-related behavioural outcome in chronic PDPN. The effect of HF DRGS and Burst DRGS and/or other DRGS settings on pain relief in PDPN animals need to be tested in an experimental model, where also operational measures (e.g., preference of location in cage due to active or placebo stimulation) are included in the final outcomes for the treatments.

At present it is not known which amplitude (:or percentage of MT in experimental models) is most adequate and effective with SCS and DRGS. A first attempt to study the effect of intensity of SCS and pain relief has been made by Meuwissen et al., ${ }^{38}$ who compared the effect of the intensity of Burst-SCS versus conventional SCS in a model of peripheral neuropathy. From this study it was concluded that Burst-SCS requires significantly more mean charge per second in order to achieve similar pain relief, as 
compared with conventional SCS. In humans, the amplitude in conventional DRGS is usually 4-10 times less (0.8-0.9 mA) than the amplitude used for conventional SCS (3.6$8.5 \mathrm{~mA}$ ). On the contrary, in the current animal study amplitudes for both SCS and DRGS were very close (67\% MT, $0.19 \pm 0.01 \mathrm{~mA}$ and $0.18 \pm 0.05 \mathrm{~mA}$, respectively). As our study was the first study to compare SCS and DRGS in an experimental animal model for PDPN, we preferred using similar settings for DRGS and SCS. However, we cannot completely exclude that with an amplitude of $67 \% \mathrm{MT}$, as in our protocol, also the dorsal columns in the spinal cord were stimulated during DRGS, thereby potentially causing a similar pain relief in the animals. The use of lower DRGS stimulation amplitudes (more like the amplitudes used clinically in humans - i.e., about $15 \%$ of the conventional output of an SCS system $)^{35}$ and effect on pain relief unquestionably needs to be tested in the near future.

\section{Limitations}

For translation of our experimental data to the clinic, where long-term SCS protocols are used for treatment of pain in PDPN, we underline that our experiments and results deal with use of short-term stimulation paradigms and that thus long-term efficacy was not studied.

\section{Conclusions}

We conclude that DRGS is as effective as SCS for PDPN associated mechanical hypersensitivity in STZ-induced diabetic rats. At the same time a faster wash-out course after cessation of the stimulation is noted with DRGS as compared to SCS. The development of the present model for DRGS in PDPN animals allows future research on mechanism and effectiveness of other clinically relevant stimulation paradigms, especially DRGS with different frequencies, lower amplitudes and longer stimulation time. 


\section{References}

1. Tesfaye S, Selvarajah D. Advances in the epidemiology, pathogenesis and management of diabetic peripheral neuropathy. Diabetes Metab Res Rev. 2012;28(SUPPL. 1):8-14.

2. Abbott CA, Malik RA, Van Ross ERE, Kulkarni J, Boulton AJM. Prevalence and characteristics of painful diabetic neuropathy in a large community-based diabetic population in the U.K. Diabetes Care. 2011;34:2220-2224.

3. Peltier A, Goutman SA, Callaghan BC. Painful diabetic neuropathy. BMJ. 2014;348(may06 1):g1799-g1799.

4. Kumar K, Toth C, Nath RK. Spinal cord stimulation for chronic pain in peripheral neuropathy. Surg Neurol. 1996;46:363-369.

5. Tesfaye S, Watt J, Benbow SJ, Pang KA, Miles J, MacFarlane IA. Electrical spinal-cord stimulation for painful diabetic peripheral neuropathy. Lancet. 1996;348:1698-1701.

6. Daousi C, SJ B, IA M. Electrical spinal cord stimulation in the long-term treatment of chronic painful diabetic neuropathy. Diabet Med. 2005;22:393-398.

7. de Vos CC, Rajan V, Steenbergen W, van der Aa HE, Buschman HP. Effect and safety of spinal cord stimulation for treatment of chronic pain caused by diabetic neuropathy. J Diabetes Complicat. 2009;23:40-45.

8. Pluijms WA, Slangen $R$, Bakkers $M$, et al. Pain relief and quality-of-life improvement after spinal cord stimulation in painful diabetic polyneuropathy: A pilot study. Br J Anaesth. 2012;109:623-629.

9. Slangen R, Pluijms WA, Faber CG, Dirksen CD, Kessels AGH, Van Kleef M. Sustained effect of spinal cord stimulation on pain and quality of life in painful diabetic peripheral neuropathy. $\mathrm{Br} J$ Anaesth. 2013;111:1030-1031.

10. Slangen R, Schaper NC, Faber CG, et al. Spinal cord stimulation and pain relief in painful diabetic peripheral neuropathy: A prospective two-center randomized controlled trial. Diabetes Care. 2014;37:3016-3024.

11. De Vos CC, Meier K, Zaalberg PB, et al. Spinal cord stimulation in patients with painful diabetic neuropathy: A multicentre randomized clinical trial. Pain. 2014;155:2426-2431.

12. Van Beek M, Slangen R, Schaper NC, et al. Sustained treatment effect of spinal cord stimulation in painful diabetic peripheral Neuropathy: 24-Month Follow-up of a prospective Two-Center randomized controlled trial. Diabetes Care. 2015;38:e132-e134.

13. Liem L, Russo M, Huygen FJPM, et al. A Multicenter, Prospective Trial to Assess the Safety and Performance of the Spinal Modulation Dorsal Root Ganglion Neurostimulator System in the Treatment of Chronic Pain. Neuromodulation. 2013:471-482.

14. Liem L, Russo M, Huygen FJPM, et al. One-Year Outcomes of Spinal Cord Stimulation of the Dorsal Root Ganglion in the Treatment of Chronic Neuropathic Pain. Neuromodulation. 2015;18:41-49.

15. Eldabe S, Espinet A, Wahlstedt A, Kang P, Liem L, Patel NK, Vesper J, Kimber A, Cusack W KJ. Retrospective case series on the treatment of painful diabetic peripheral neuropathy with dorsal root ganglion stimulation. Neuromodulation. 2018;Mar 25. [Epub ahead of print]

16. Galer BS, Gianas A, Jensen MP. Painful diabetic polyneuropathy: Epidemiology, pain description, and quality of life. Diabetes Res Clin Pract. 2000;47:123-128.

17. Pluijms W A, van Kleef $M$, Honig WM, Janssen SP, Joosten $E$ A. The effect of spinal cord stimulation frequency in experimental painful diabetic polyneuropathy. Eur J Pain. 2013;17:1338-1346.

18. Pan B, Yu H, Fischer GJ, Kramer JM, Hogan QH. Dorsal Root Ganglionic Field Stimulation Relieves Spontaneous and Induced Neuropathic Pain in Rats. J Pain. 2016;17:1349-1358.

19. Calcutt N a. Modeling diabetic sensory neuropathy in rats. Methods Mol Med. 2004;99:55-65.

20. Chaplan SR, Bach FW, Pogrel JW, Chung JM, Yaksh TL. Quantitative assessment of tactile allodynia in the rat paw. J Neurosci Methods. 1994;53:55-63.

21. Beek $M$, Kleef $M$, Linderoth $B$, et al. Spinal cord stimulation in experimental chronic painful diabetic polyneuropathy: Delayed effect of High-frequency stimulation. Eur J Pain. 2017;21:795-803.

22. Mills C, Leblond D, Joshi S, et al. Estimating efficacy and drug ED50's using von frey thresholds: Impact of Weber's Law and log transformation. J Pain. 2012;13:519-523. 


\section{Chapter 4}

23. Guastella V, Mick G. Strategies for the diagnosis and treatment of neuropathic pain secondary to diabetic peripheral sensory polyneuropathy. Diabetes Metab. 2009;35:12-19.

24. Mailis-Gagnon A, Furlan A, Sandoval J, Taylor R. Spinal cord stimulation in chronic pain. Cochrane Database Syst Rev. 2004;32:11-21.

25. Linderoth B, Foreman RD. Conventional and Novel Spinal Stimulation Algorithms: Hypothetical Mechanisms of Action and Comments on Outcomes. Neuromodulation. 2017;20:525-533.

26. Yakhnitsa V, Linderoth B, Meyerson BA. Spinal cord stimulation attenuates dorsal horn neuronal hyperexcitability in a rat model of mononeuropathy. Pain. 1999;79:223-233.

27. Chang Chien GC, Mekhail N. Alternate Intraspinal Targets for Spinal Cord Stimulation: A Systematic Review. Neuromodulation. 2017;20:629-641.

28. Sapunar D, Kostic S, Banozic A, Puljak L. Dorsal root ganglion - A potential new therapeutic target for neuropathic pain. J Pain Res. 2012;5:31-38.

29. McCallum JB, Kwok W-M, Sapunar D, Fuchs A, Hogan QH. Painful peripheral nerve injury decreases calcium current in axotomized sensory neurons. Anesthesiology. 2006;105:160-168.

30. Rush AM, Dib-Hajj SD, Liu S, Cummins TR, Black JA, Waxman SG. A single sodium channel mutation produces hyper- or hypoexcitability in different types of neurons. Proc Natl Acad Sci. 2006;103:8245-8250.

31. Devor M. Neuropathic pain: What do we do with all these theories? Acta Anaesthesiol Scand. 2001;45:1121-1127.

32. Miller JP, Eldabe S, Buchser E, Johanek LM, Guan Y, Linderoth B. Parameters of Spinal Cord Stimulation and Their Role in Electrical Charge Delivery: A Review. Neuromodulation. 2016;19:373-384.

33. Geurts JW, Joosten EA, Van Kleef M. Current status and future perspectives of spinal cord stimulation in treatment of chronic pain. Pain. 2017;158:771-774.

34. Vos C, Meier K, Zaalberg $P$, et al. Spinal cord stimulation in patients with painful diabetic neuropathy: a multicentre randomized clinical trial. Pain. 2014;155:2426-2431.

35. Deer TR, Grigsby E, Weiner RL, Wilcosky B, Kramer JM. A prospective study of dorsal root ganglion stimulation for the relief of chronic pain. Neuromodulation. 2013;16:67-72.

36. Van Buyten J-P, Smet I, Liem L, Russo M, Huygen F. Stimulation of Dorsal Root Ganglia for the Management of Complex Regional Pain Syndrome: A Prospective Case Series. Pain Pract. 2014;15:208-216.

37. Schu S, Gulve A, Eldabe S, et al. Spinal Cord Stimulation of the Dorsal Root Ganglion for Groin Pain-A Retrospective Review. Pain Pract. 2014;15:293-299.

38. Meuwissen KPV, Gu JW, Zhang TC, Joosten EAJ. Conventional-SCS vs. Burst-SCS and the Behavioral Effect on Mechanical Hypersensitivity in a Rat Model of Chronic Neuropathic Pain: Effect of Amplitude. Neuromodulation. 2018;21:19-30. 


\section{Chapter 5}

\section{Dorsal Root Ganglion Stimulation in Experimental Painful Diabetic Polyneuropathy: Delayed Wash-out of Pain Relief after Low-Frequency $(1 \mathrm{~Hz})$ Stimulation}

Eva Koetsier, MD, ${ }^{1}$ Glenn Franken, $\mathrm{MSc}^{2,3}$ Jacques Debets, ${ }^{4}$ Sander M.J. van Kuijk $\mathrm{PhD},{ }^{5}$ Bengt Linderoth, PhD, MD, ${ }^{6}$ Elbert A. Joosten, PhD, ${ }^{2,3}$ Paolo Maino, $\mathrm{MD}^{1}$

${ }^{1}$ Pain Management Center, Neurocenter of Southern Switzerland, Regional Hospital of Lugano, Via Capelli, 6962, Lugano, Switzerland

${ }^{2}$ Department of Anesthesiology and Pain Management, Maastricht University Medical Center ${ }^{+}$, Maastricht, the Netherlands

${ }^{3}$ Department of Translational Neuroscience, School of Mental Health and Neuroscience (MHeNS), University of Maastricht, the Netherlands

${ }^{4}$ Muroidean Facility, School of Cardiovascular Diseases (CARIM), Maastricht, the Netherlands

${ }^{5}$ Department of Clinical Epidemiology and Medical Technology Assessment, Maastricht University Medical Center ${ }^{+}$, Maastricht, the Netherlands.

${ }^{6}$ Department of Clinical Neuroscience, Karolinska Institutet, SE-171 77 Stockholm, Sweden

Neuromodulation. 2019;23:177-184. 


\section{Abstract}

Objective: Up till now there is little data about the pain relieving effect of different frequency settings in DRGS. The aim of this study was to compare the pain relieving effect of DRGS at low-, mid- and high-frequencies and Sham-DRGS in an animal model of painful diabetic neuropathy (PDPN).

Material and Methods: Diabetes mellitus was induced by an intraperitoneal injection of streptozotocin in 8-week-old female Sprague-Dawley rats ( $n=24$; glucose $\geq 15 \mathrm{mmol} / \mathrm{L}: n$ = 20; mechanical hypersensitivity: $n=15$ ). Five weeks later, a DRGS device was implanted at the L5 DRG. Ten animals were included for stimulation, alternating 30 minutes of low(1 $\mathrm{Hz})-$, $\operatorname{mid}(20 \mathrm{~Hz})-$ and high $(1000 \mathrm{~Hz})$-frequencies and Sham-DRGS during four days, with a pulse width of $0.2 \mathrm{msec}$ (average amplitude: $0.19 \pm 0.01 \mathrm{~mA}$ ), using a randomized crossover design. The effect on mechanical hypersensitivity of the hind paw to von Frey filaments was evaluated.

Results: All DRGS frequencies resulted in a complete reversal of mechanical hypersensitivity and "a clinically relevant reduction" was achieved in 70-80\% of animals. No significant differences in maximal pain relieving effect were found between the different frequency treatments $(p=0.24)$. Animals stimulated at $1000 \mathrm{~Hz}$ and $20 \mathrm{~Hz}$ returned to baseline mechanical hypersensitivity values $15 \mathrm{~min}$ and $30 \mathrm{~min}$ after stimulation cessation, respectively, while animals stimulated at $1 \mathrm{~Hz}$ did not.

Conclusion: These results show that DRGS is equally effective when applied at low-, midand high-frequency in an animal model of PDPN. However, low-frequency-(1Hz)-DRGS resulted in a delayed wash-out effect, which suggests that this is the most optimal frequency for pain therapy in PDPN as compared to mid-and high-frequency. 


\section{Introduction}

Diabetes is a worldwide epidemic, and associated painful diabetic neuropathy (PDPN) is its most costly and disabling complication. ${ }^{1}$ Unfortunately, pharmacological pain therapy is often insufficient and side effects can be intolerable. ${ }^{2,3}$ Spinal cord stimulation (SCS) is a therapy for chronic pain, in which epidurally implanted electrodes stimulate primary afferent $(A \beta)$ collaterals in the dorsal columns. SCS has resulted in significant pain reduction in PDPN patients. ${ }^{4-6}$ Nevertheless, in about $40 \%$ of the patients the treatment effect is not sufficient. ${ }^{4-6}$ Dorsal Root Ganglion Stimulation (DRGS) has more recently been successfully applied for pain relief in several clinical trials ${ }^{7-9}$ and is a promising neuro-stimulation modality in the treatment of PDPN ${ }^{10}$, also demonstrated in preclinical studies. ${ }^{11}$ While SCS is often unable to cover difficult-to-reach areas (e.g. the groins, the feet) without generating extensive paresthesias or motor side effects, DRGS has been shown to be able to cover these difficult-to-reach areas. ${ }^{8}$ A retrospective case series suggested that conventional DRGS improves painful symptoms in PDPN patients in particular. ${ }^{10}$ Optimization of the stimulation frequency might result in additional beneficial effects in experimental PDPN. The results of a recently published experimental study ${ }^{11}$ showed that conventional DRGS $(50 \mathrm{~Hz})$ is equally effective as conventional SCS (40-50 Hz) in reduction of PDPN associated mechanical hypersensitivity in experimental PDPN. Both SCS and DRGS induced a complete reversal of mechanical hypersensitivity, which was obtained after 15 minutes in the SCS group versus after 30 minutes in the DRGS group. Despite these positive results, there is still room for improvement regarding in particular the rate of responders. As the concept underlying DRGS differs from SCS, it is of the utmost importance to test various stimulation settings of DRGS in an experimental PDPN model. Up till now there is little data about the pain relieving effect of different frequency settings in DRGS and (experimental and clinical) trials assessing the efficacy of different frequencies for DRGS beside the conventional DRGS frequency are indicated. The present study was aimed to compare the pain relieving effect of DRGS at $1 \mathrm{~Hz}$ (defined as 'low-frequency', LF), at $20 \mathrm{~Hz}$ (defined as 'mid-frequency', MF), at $1000 \mathrm{~Hz}$ (defined as 'high-frequency', HF) and Sham-DRGS in an animal model of PDPN.

\section{Materials and methods}

\section{Animals}

The experiments for this study were performed using 24 female Sprague-Dawley rats, which were 8 weeks of age and weighing 170-240 g at the beginning of the study (Charles River, Maastricht, the Netherlands). Animals were housed in pairs before DRGS implantation and individually after surgery in a climate controlled room with a 12-h reversed light dark cycle in transparent plastic cages with free access to food and water. 
The study was approved by the Animal Research Committee of the Maastricht University (DEC-protocol 2017-022) and is in accordance with the guidelines of the European Directive for the Protection of Vertebrate Animals Used for Experimental and Other Scientific Purposes (86/609/EU). Every effort was made to keep animal numbers as low as possible and to minimize their suffering. Alternatives to in vivo techniques were considered.

\section{Induction of diabetes mellitus}

After 1 night of fasting, diabetes mellitus (DM) was induced by intraperitoneal injection of $65 \mathrm{mg} / \mathrm{kg}$ Streptozotocin (STZ,65 mg/mL, Sigma-Aldrich, Schnelldorf, Germany). One week after STZ injection ( $n=24)$, blood glucose levels were measured in blood derived from the saphenous vein with a standard blood glucose meter (Accu-Chek Aviva ${ }^{\circledR}$, Roche Diagnostics $\mathrm{GmbH}$, Mannheim, Germany) to verify the induction of DM (blood glucose level $\geq 15 \mathrm{mmol} / \mathrm{L}$ ). Rats with a glucose level of $\geq 15 \mathrm{mmol} / \mathrm{L}$ were considered diabetic ${ }^{12}$ and were included in the study.

\section{Behavioral testing}

Animals were tested for mechanical hypersensitivity based on the hind limb paw withdrawal response to von Frey filaments (bending forces 0.6, 1.2 2 2.0, 3.6, 5.5, 8.5 , 15.1, and $28.84 \mathrm{~g}$ ). Before start of the behavioral testing, rats were placed in the experimental set-up and were able to acclimatize for $15 \mathrm{~min}$. Mechanical hypersensitivity was assessed according to the 'up-down method', ${ }^{13}$ which was previously described. ${ }^{14}$ To prevent tissue damage, the absence of the paw withdrawal response to a $28.84 \mathrm{~g}$ force was determined as the cut-off value. The registered $50 \%$ paw withdrawal thresholds (WT), measured in grams, were multiplied by 10.000 and then logarithmically transformed in order to conform with Weber's law ${ }^{15}$ and to obtain a linear scale.

\section{Inclusion of animals}

At baseline (pre-STZ injection= pre-diabetes) $(n=24)$, as well as at 4 weeks post-STZ injection, animals were tested for mechanical hypersensitivity using von Frey hind limb withdrawal testing. The presence of mechanical hypersensitivity was presumed if the $\log ^{10}(10.000 \times 50 \%$ WT $)$ decreased by $\geq 0.2$ units compared with baseline. ${ }^{13,16}$ Only animals showing mechanical hypersensitivity at 4 weeks post-STZ injection $(n=15)$ were treated with DRGS, whereas animals showing no mechanical hypersensitivity at that time point were excluded from the study $(n=5)$.

\section{Implantation of DRGS device}

A bipolar DRGS electrode (Fig. 1) was implanted unilaterally at the L5 DRG, according the technique that was previously described (adapted from Pan et al.). ${ }^{11,17}$ In short, the L5 
intervertebral foramen was exposed under general anaesthesia, and both the anode and cathode were implanted at the DRG. The electrode was secured on the transverse process, and the incision was closed.The animals recovered for 2 days after the DRGS implantation.

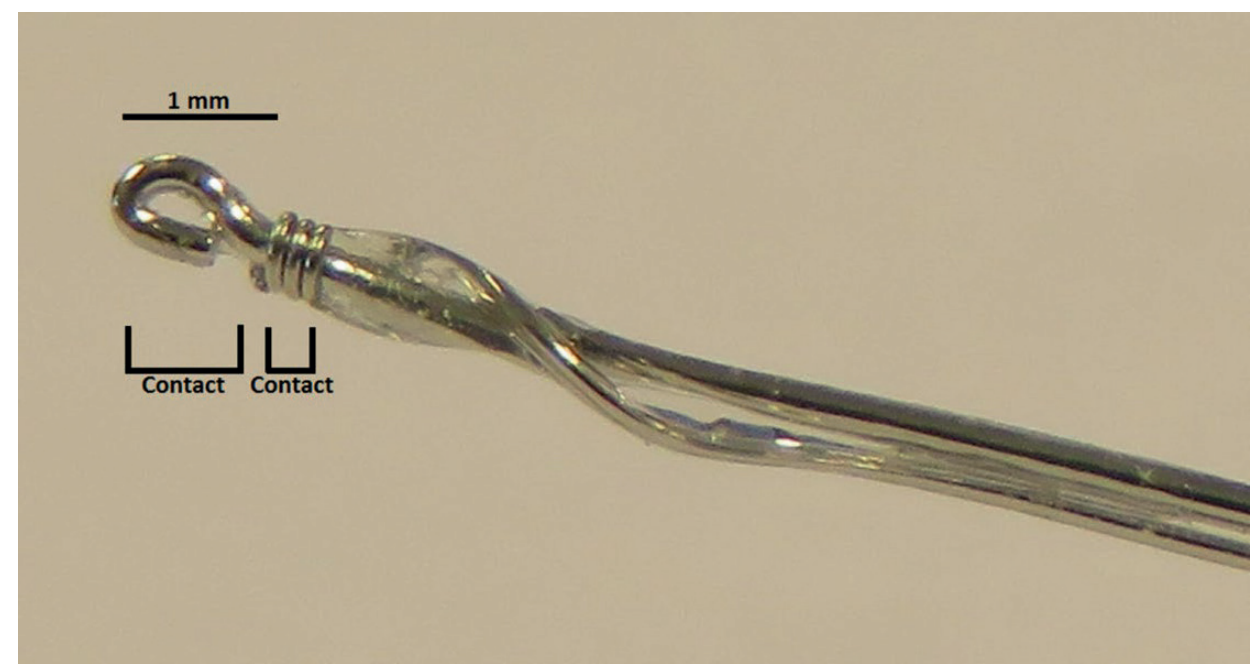

Figure 1. Bipolar electrode for dorsal root ganglion stimulation.

\section{DRGS}

All animals received LF-DRGS of $1 \mathrm{~Hz}$, MF-DRGS of $20 \mathrm{~Hz}$, HF-DRGS of $1000 \mathrm{~Hz}$ and ShamDRGS at days 2-5 post-implantation, using a randomized cross-over design. After the connector was attached to the wire of the pulse generator (A-M systems MultiStim Model 3800, fitted with an A-M systems 3820 stimulus isolator (A-M systems, Sequim, WA, USA)), the assessment of motor threshold (MT) was performed by progressively augmenting the current amplitude using a constant frequency of $2 \mathrm{~Hz}$ and a pulse width of $0.2 \mathrm{msec}$. The current amplitude that induced contractions in the lower limb in the animal was defined to be the MT for that specific animal. On stimulation days, animals were first tested for $\mathrm{MT}$, after which the amplitude was set accordingly for each animal (with stimulation amplitude at $66.7 \%$ of $\mathrm{MT}$ - defined as above). Biphasic stimulation was applied for 30 minutes, alternating LF- $(1 \mathrm{~Hz}), \mathrm{MF}-(2 \mathrm{~Hz}), \mathrm{HF}-(1000 \mathrm{~Hz})$ and Sham-DRGS during the four consecutive days, with a pulse width of $0.2 \mathrm{msec}$ (average amplitude: 0.19 $\pm 0.01 \mathrm{~mA})$. 
Animals were tested for mechanical hypersensitivity immediately before stimulation (pre-stimulation baseline) ( $=2-5$ days after implantation), during stimulation at 15 ( $\mathrm{t}=15$ $\mathrm{min}$ ) and at $30 \mathrm{~min}(\mathrm{t}=30 \mathrm{~min})$, and after stimulation at 15 and $30 \mathrm{~min}(\mathrm{t}=45 \mathrm{~min}$ and $\mathrm{t}=60$ min) (Fig. 2). An animal was defined as a 'responder to stimulation' if the $\log ^{10}(10.000 x$ $50 \%$ WT) increased with $\geq 0.2$ during stimulation, when compared to the pre-stimulation baseline. ${ }^{11}$

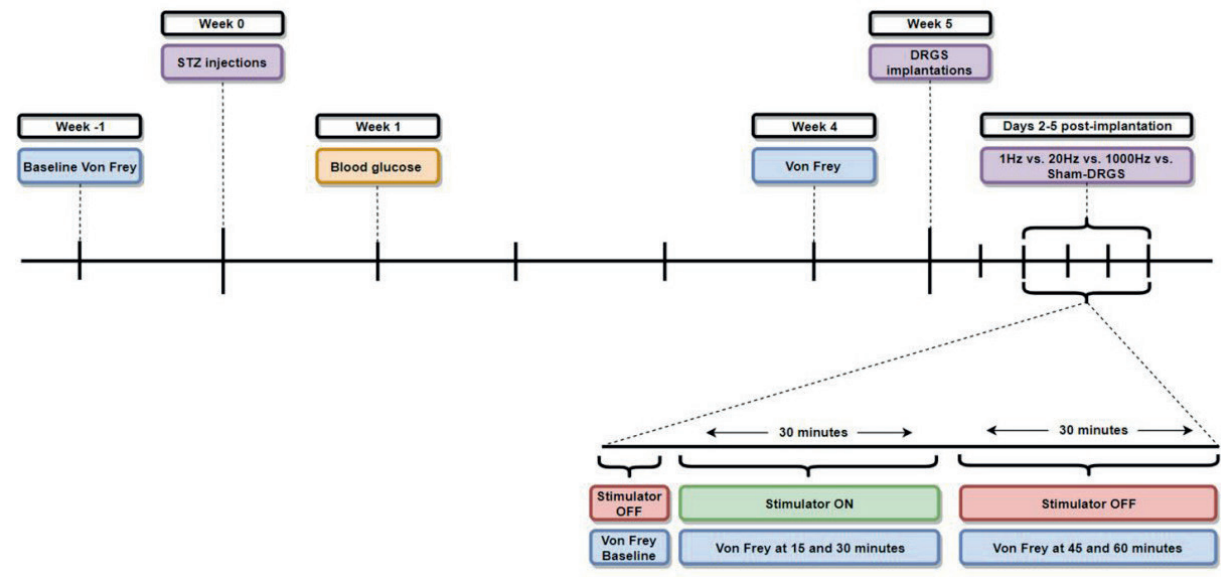

Figure 2. Study design.

DRGS, Dorsal Root Ganglion Stimulation.

\section{Statistical analysis}

All data are represented as mean \pm standard error of the mean (SEM). For comparison of mechanical hypersensitivity pre-STZ injection (=pre-diabetes) and pre-implantation, a paired t-test was used. For comparison of the effect of DRGS over time and the effect of stimulation frequency, a two-way analysis of variance (ANOVA) followed by Dunnet's or Tukey's post-hoc test was used, respectively. For comparisons of MTs over time, a repeated measures one-way ANOVA followed by Dunnett's multiple comparison test was used. Statistical significance was defined as $p<0.05$. 


\section{Results}

\section{Description of cohorts of animals}

Out of the 24 STZ-injected animals, 20 animals developed DM (83\%; blood glucose level $\geq 15 \mathrm{mmol} / \mathrm{L}$ ). Fifteen out of these 20 diabetic animals developed PDPN (75\%; $\geq 0.2$ decrease in $\log ^{10}(10.000 \times 50 \%$ WT $)$ on von Frey when compared to the pre-diabetes level). The mean $\log ^{10}(10.000 \times 50 \% \mathrm{WT})$ in these animals dropped from $5.19 \pm 0.04 \mathrm{~g}$ at pre-diabetes-baseline to $4.64 \pm 0.05 \mathrm{~g}$ four weeks post-STZ injection $(P<0.0001)$ (Fig. 3). All PDPN animals $(n=15)$ were then implanted with a unilateral DRGS device at the L5 lumbar level. One animal died during implantation. Out of the 14 implanted animals, 2 animals were excluded due to excessively high $\mathrm{MT}(\geq 1 \mathrm{~mA})$, and 2 animals were excluded due to not being able to complete the full 4-days stimulation protocol (lead defects).

\section{Development of PDPN}

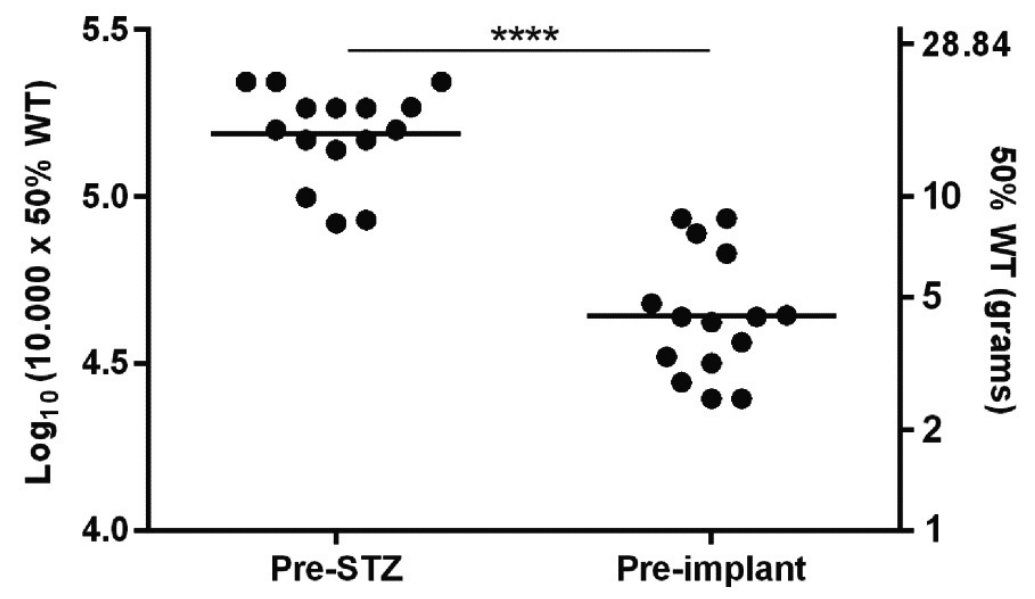

Figure 3. Development of PDPN. PDPN was defined as a $\geq 0.2$ decrease in $\log ^{10}(10.000 \times 50 \%$ WT $)$ on von Frey. **** $p<0.0001$.

PDPN, painful diabetic polyneuropathy; WT, withdrawal threshold; STZ, streptozotocin.

Effect over time of DRGS at $1 \mathrm{~Hz}, 20 \mathrm{~Hz}, 1000 \mathrm{~Hz}$ and Sham-DRGS on mechanical hypersensitivity

DRGS significantly attenuated STZ-induced PDPN ( $p<0.0001$, effect of factor time; twoway repeated measures ANOVA). Scatter plots with individual animal performances for $1 \mathrm{~Hz}(\mathrm{LF}), 20 \mathrm{~Hz}$ (MF), $1000 \mathrm{~Hz}$ (HF) and Sham-DRGS are presented in Figure 4A, 4B, 4C, 4D, respectively. In the LF-DRGS treatment, DRGS resulted in an increase of the $\log ^{10}$ (10 000 $\times 50 \%$ WT) from $4.55 \pm 0.10$ at baseline (pre-DRGS) to $4.94 \pm 0.14$ at $15 \mathrm{~min}(p<0.01)$ and 
$5.15 \pm 0.12$ at $30 \mathrm{~min}(p<0.0001)$. After cessation of LF-DRGS, the $\log ^{10}(10000 \times 50 \% W T)$ did not return to pre-DRGS values at $45 \min (4.98 \pm 0.14 ; p<0.001)$ and $60 \min (4.91 \pm$ $0.12 ; \mathrm{p}<0.01$ ) (Fig. $4 \mathrm{a}$ and Fig. 4e). In the MF-DRGS treatment, DRGS resulted in an increase of the $\log ^{10}(10000 \times 50 \%$ WT $)$ from $4.62 \pm 0.15$ pre-DRGS to $5.10 \pm 0.13$ at 15 $\min (p<0.0001)$ and $5.12 \pm 0.10$ at $30 \mathrm{~min}(p<0.0001)$. After DRGS was stopped, the $\log ^{10}$ $(10000 \times 50 \% \mathrm{WT})$ did not return to pre-DRGS values at $45 \mathrm{~min}(4.92 \pm 0.16 ; p<0.05)$, but did however return to pre-DRGS at $60 \mathrm{~min}(4.79 \pm 0.13$; $\mathrm{p}=0.29$ ) (Fig. $4 \mathrm{~b}$ and Fig. $4 \mathrm{e}$ ). In the HF-DRGS treatment, DRGS resulted in an increase of the $\log ^{10}(10000 \times 50 \%$ WT $)$ from $4.62 \pm 0.12$ pre-DRGS to $5.18 \pm 0.11$ at $15 \mathrm{~min}(\mathrm{p}<0.0001)$ and $5.20 \pm 0.12$ at $30 \mathrm{~min}$ $(p<0.0001)$. After cessation of DRGS, the $\log ^{10}(10000 \times 50 \% \mathrm{WT})$ returned to pre-DRGS values at $45 \min (4.87 \pm 0.10 ; p=0.06)$, and at $60 \min (4.80 \pm 0.12 ; p=0.26)$ (Fig. $4 c$ and Fig. $4 \mathrm{e})$. In the Sham-DRGS treatment, no increases of the $\log ^{10}(10000 \times 50 \%$ WT $)$ compared to pre-DRGS values (4.73 \pm 0.10$)$ were found at $15 \mathrm{~min}(4.68 \pm 0.12 ; p=0.98)$, at $30 \min (4.69 \pm 0.11 ; p=0.99), 45 \min (4.73 \pm 0.09 ; p=0.99)$ and at $60 \min (4.77 \pm 0.10$; $p=0.99$ ) (Fig. 4d and Fig. 4e).

All treatment modalities (LF-, MF-, and HF-DRGS) resulted in a return to pre-diabetes injection $\log ^{10}(10000 \times 50 \% W T)$ values $(p>0.07)$. No significant differences were observed in terms of effect of stimulation frequency between groups ( $p=0.24$, effect of factor frequency; two-way repeated measures ANOVA). At $t=15 \mathrm{~min}$, the MF $(p<0.001)$, and HF- $(p<0.0001)$ DRGS scored significantly better in terms of mechanical hypersensitivity compared to Sham-DRGS (two-way repeated measures ANOVA followed by Tukey's multiple comparisons test). Interestingly, the $1 \mathrm{~Hz}$ group showed no significant difference compared to the Sham group at $15 \mathrm{~min}(p=0.07)$. At $30 \mathrm{~min}$ of stimulation, the LF- $(p<0.001)$, MF- $(p<0.001)$, and HF-DRGS $(p<0.0001)$ scored significantly better in terms of mechanical hypersensitivity as compared to the Sham-DRGS (two-way repeated measures ANOVA followed by Tukey's multiple comparisons test) (Fig. 4e). No significant differences between frequencies were observed at any other time point (inter-treatment comparison, two-way repeated measures ANOVA followed by Tukey's multiple comparisons test). 

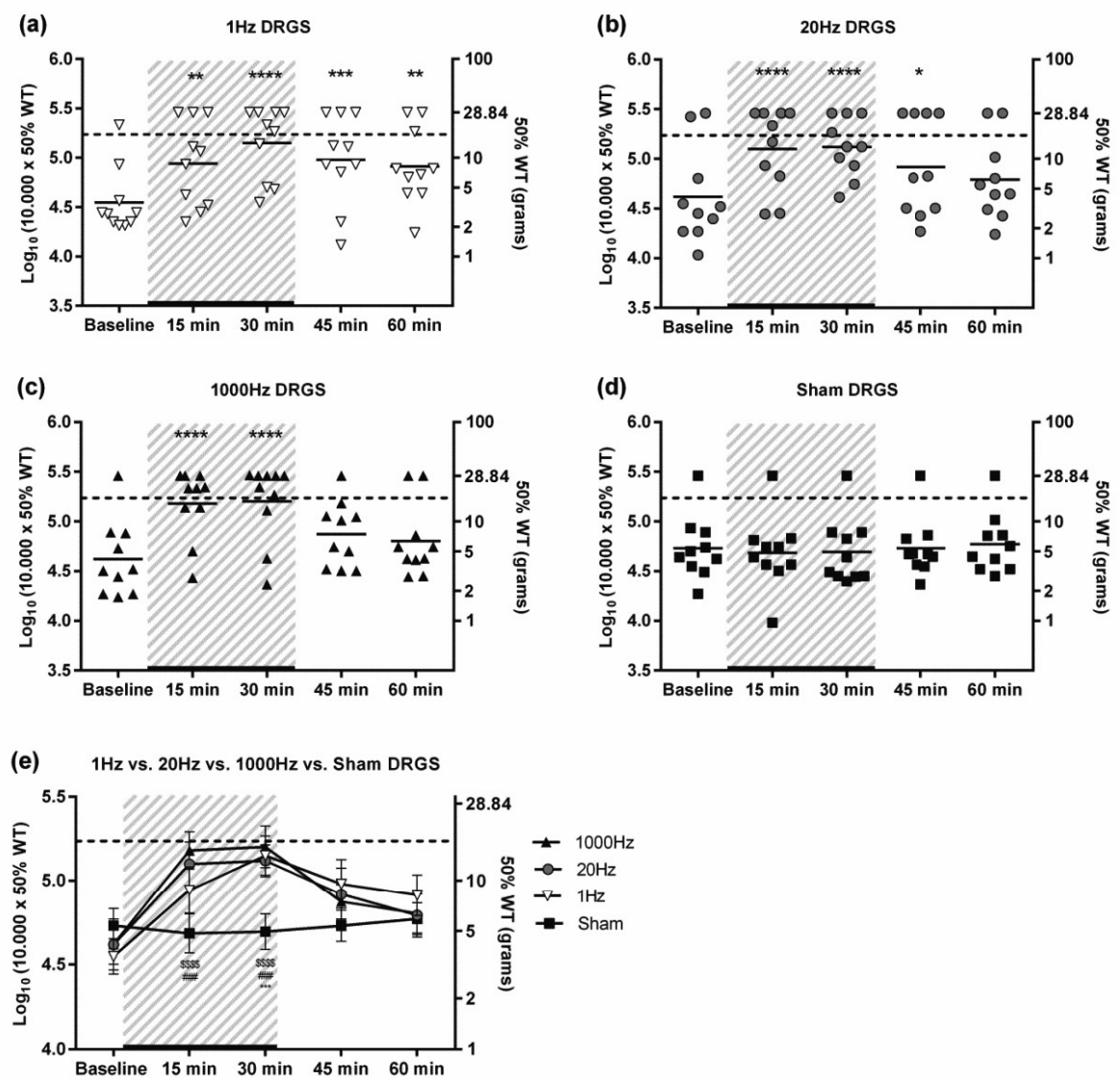

Figure 4a-d. Scatter plot for the effect of the different frequencies and Sham-DRGS on mechanical hypersensitivity of the left hind paw in rats with PDPN. Dotted line $=$ mean $\log ^{10} 50 \%$ WT before STZ injection. Stimulator is on at the 15 and 30 min time point. ${ }^{*} p<0.05,{ }^{*} P<0.01,{ }^{*} * * p<0.001,{ }^{*} * * * p<0.0001$ when compared to baseline (two-way repeated measures ANOVA followed by Dunnett's multiple comparisons correction). (a) Scatter plot for the effect of LF- DRGS on mechanical hypersensitivity. (b) Scatter plot for the effect of MF- DRGS on mechanical hypersensitivity. (c). Scatter plot for the effect of HF-DRGS on mechanical hypersensitivity. (d) Scatter plot for the effect of Sham DRGS on mechanical hypersensitivity. No stimulation applied. (e) Effect of DRGS using different stimulation frequencies on mechanical hypersensitivity. Data are presented as mean \pm SEM. ${ }^{*} p<0.05$ compared to LF-DRGS $* * * p<0.001$ compared to LF-DRGS. \#\#\#p<0.001 compared to MF-DRGS. $\$ \$ \$ p<0.0001$ compared to $1000 \mathrm{~Hz}$ treatment. Hz, Hertz, DRGS, dorsal root ganglion stimulation; WT, withdrawal threshold; min, minutes; LF, low-frequency; MF, mid-frequency, HF, highfrequency. 
Chapter 5

\section{Percentage responders}

The percentage of animals responding to DRGS at 15 min was highest during HFstimulation (8/10 (80\%)) and at $30 \mathrm{~min}$ it was highest during the HF and LF-stimulation (both $8 / 10(80 \%)$ ). At 45 and 60 min (i.e. after stimulation OFF), responder rates were considerably higher with use of LF stimulation when compared to the other frequencies of DRGS (Table 1).

Table 1. Responder rates for each DRGS frequency.

\begin{tabular}{lllll}
\hline Treatment & $\mathbf{T = 1 5} \mathbf{m i n}$ & $\mathbf{T = 3 0} \mathbf{m i n}$ & $\mathbf{T = 4 5} \mathbf{m i n}$ & $\mathbf{T = 6 0} \mathbf{m i n}$ \\
\hline $\mathbf{1 H z}$ & $5 / 10(50 \%)$ & $8 / 10(80 \%)$ & $7 / 10(70 \%)$ & $7 / 10(70 \%)$ \\
$\mathbf{2 0 H z}$ & $6 / 10(60 \%)$ & $7 / 10(70 \%)$ & $5 / 10(50 \%)$ & $3 / 10(30 \%)$ \\
$\mathbf{1 0 0 0 H z}$ & $8 / 10(80 \%)$ & $8 / 10(80 \%)$ & $5 / 10(50 \%)$ & $5 / 10(50 \%)$ \\
Sham & $1 / 10(10 \%)$ & $0 / 10(0 \%)$ & $1 / 10(10 \%)$ & $1 / 10(10 \%)$ \\
\hline
\end{tabular}

A responder to stimulation was defined as an animal with an increase of the $\log 10(10.000 \times 50 \%$ WT) $\geq 0.2$ compared to the pre-stimulation baseline. $\mathrm{Hz}$, Hertz; min, minutes.

Lastly, a small increase over time in terms of MTs was observed over the 4-day stimulation protocol ( $p<0.05$, repeated measures one-way ANOVA) (Fig. 5).

\section{Motor threshold}

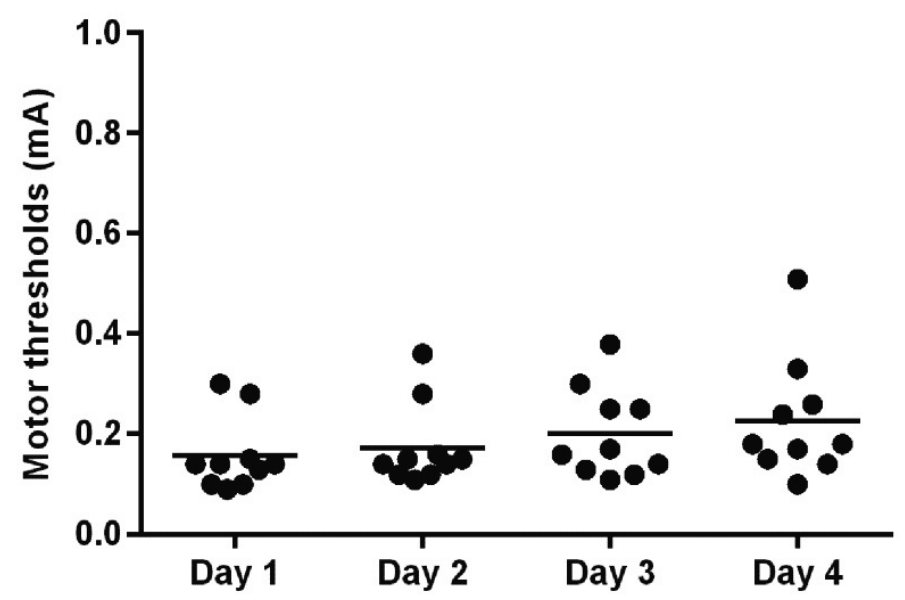

Figure 5. Observed motor thresholds over time. Data are presented as scatter plots + mean. ${ }^{*} p<0.05$. $\mathrm{mA}$, milliampere. 


\section{Discussion}

With this study, we aimed to compare the behavioral anti-nociceptive effect of the conventionally used MF-DRGS with LF-DRGS, HF-DRGS and Sham-DRGS on mechanical hypersensivity in an animal model of the acute phase of PDPN. The results of this study show that DRGS is equally effective when applied at LF $(1 \mathrm{~Hz}), \mathrm{MF}(20 \mathrm{~Hz})$ and $\mathrm{HF}(1000 \mathrm{~Hz})$ in an animal model of PDPN. However, LF-DRGS resulted in a delayed wash-out effect. These findings suggest that LF-DRGS results in an extended duration of pain relief and with that is most optimal for pain therapy in PDPN as compared to MF and HF.

The vasculature system plays a major role in the pathophysiology of PDPN and SCS has been suggested to induce vasodilation which improves peripheral blood flow. ${ }^{18}$ Previous studies showed that SCS at higher frequencies $(500 \mathrm{~Hz}$ and $200 \mathrm{~Hz})$ significantly increased cutaneous blood flow in comparison to changes induced by lower frequencies. ${ }^{19,20}$ It is reasonable to assume that this vasodilating effect of SCS might also occur with the application of DRGS. Therefore, one could hypothesize that DRGS with higher frequencies also leads to superior pain relief and a higher rate of responders in comparison to DRGS with lower frequencies. However, although at 15 min HF-DRGS led to higher responder rates compared to MF-DRGS and LF-DRGS ( $80 \%$ versus $60 \%$ and $50 \%$, respectively), HF-DRGS did not lead to a significant superior pain relieving effect. DRGS at all three frequencies (LF, MF and HF) resulted in reversal of mechanical hypersensitivity and a "clinically relevant reduction" was achieved in 70-80\% of animals at $30 \mathrm{~min}$. At 15 min, the LF-DRGS treatment was not significantly different compared to Sham-DRGS, whereas this was the case for the MF-DRGS and HF-DRGS. This result might hint to a delayed onset of the effect with the lower frequency DRGS treatment. A small increase over time in terms of MTs was observed over the 4-day stimulation protocol, which is comparable to other studies.

In the present study, the DRGS mid-frequency was set at $20 \mathrm{~Hz}$, which is the frequency most frequently used for DRGS in humans. 7,8,17,21,22 In previous DRGS-animal studies frequencies of $20-50 \mathrm{~Hz}$ were used, which resulted in a significant reversal of neuropathic pain behavior. ${ }^{11,17}$ Until now there is little data about the use of HF for DRGS. Nevertheless, one case report ${ }^{23}$ and one pilot study ${ }^{24}(\mathrm{n}=5)$ applying HF-DRGS $(2-10 \mathrm{kHz})$ in the treatment of chronic back pain, have shown positive results.

Conventionally, SCS of the dorsal columns and DRGS frequencies vary between 20 and $80 \mathrm{~Hz}$. 7,8,21,25,26 In SCS, the effect of different frequencies on pain relief in PDPN-animals was tested at lower frequencies of $4.0-10 \mathrm{~Hz}$, middle frequencies of $35-55 \mathrm{~Hz}$ and higher frequencies varying between $150-375 \mathrm{~Hz}$ in the acute phase of the disease. ${ }^{16}$ From this study it was concluded that SCS is equally effective on mechanical hypersensitivity when applied at $4.0-10 \mathrm{~Hz}, 35-55 \mathrm{~Hz}$ or $150-375 \mathrm{~Hz}$. Interestingly, use of SCS at LF $(4.0-10 \mathrm{~Hz})$ did not result in a delayed wash-out of analgesia after cessation of stimulation. ${ }^{16}$ As we did on the other hand observe a delayed wash-out effect after LF-DRGS in the current study, this effect may be related to the location of stimulation. In the chronic phase of 
experimental PDNP, the use of a high SCS frequency $(500 \mathrm{~Hz})$ resulted in a delayed and somewhat extended - but not superior effect on mechanical hypersensitivity as compared to lower frequencies SCS ( 5 and $50 \mathrm{~Hz}){ }^{14}$

The DRG contains the somata for all sensory neuron subtypes, not only the nonnociceptive $A \beta$ cell bodies but also the $A \delta$ and $C$-type nociception related cell bodies. With SCS the $A \beta$ fibers in the dorsal columns are stimulated and not the $A \delta$ or $\mathrm{C}$-fibers ${ }^{27}$, whereas with DRGS it is reasonable to suggest that all cell bodies are stimulated. Furthermore, an experimental study by Sandkühler et al. showed that LF-DRGS of $1 \mathrm{~Hz}$ of A $\delta$-type fibers resulted in the long-term depression (LTD) of the nociceptive transmission in the dorsal horn. ${ }^{28}$ From this we suggest that that the delayed wash-out effect noted with LF-DRGS is related to the induction of an A $\delta$ mediated LTD process in the dorsal horn.

Detailed understanding of how SCS and/or DRGS inhibit chronic neuropathic pain is limited. The Gate Control Theory provides a supposed mechanism of action for SCS: stimulating myelinated $A \beta$-fiber collaterals in the dorsal columns activates inhibitory GABA-ergic interneurons in the dorsal horn that suppress the transmission of nociceptive information from wide-dynamic range (WDR) spinal projection neurons to the brain. ${ }^{29-35}$ On the other hand, DRGS is suggested to amplify the natural filtering process that limits propagation of trains of action potentials through the T-junction in the DRG. ${ }^{27,36} \mathrm{An}$ experimental animal study was able to show with use of functional magnetic resonance imaging ( $\mathrm{FMRI}$ ) that DRGS in animals with chronic neuropathic pain caused a significant attenuation of the blood oxygen-level dependent (BOLD) response to noxious stimulation in brain regions associated with sensory as well as cognitive-motivational aspects of pain. ${ }^{37}$ This BOLD signal attenuation persisted for 20 minutes after the DRGS was discontinued. Although the role of GABA-ergic signaling in the mechanism of SCS has been established, the role of spinal dorsal horn GABA in DRGS-induced pain suppression needs still to be elucidated.

Limitations of this study include fundamental differences in the clinical and preclinical manifestation of PDPN. In patients, DM is a chronic disease where complications often only arise after many years. STZ injection in rats relies on destruction of beta cells in the pancreas. ${ }^{12}$ The injection therefore leads to a very swift development of DM (within a week), and subsequent development of mechanical hypersensivity (within 4-5 weeks) 11,16,38,39 Therefore, one should be cautious when extrapolating these results to a human situation. Secondly, it should be noted that the experiments as described are basically using short-term stimulation paradigms. This is up till now standard procedure in experimental studies, but it should be acknowledged that the translational impact of these studies might be limited as in clinic long-term stimulation is used. It might furthermore also be possible that use of long-term DRGS-stimulation paradigms might result into different outcomes. Van Beek et al. recently demonstrated that long-term SCS (of four weeks, 12 hours/day) resulted in decreased baseline mechanical hypersensitivity in a rat model of PDPN, suggesting an improvement in the baseline condition with long- 
term SCS treatment. ${ }^{38}$ Future experiments should be performed with longer-term DRGSstimulation paradigms which much more closely mimics the clinical situation.

\section{Conclusion}

To conclude, the results of the present study show: 1.) the maximal anti-nociceptive effect based on paw withdrawal responses did not differ between LF-, MF- and HF-DRGS; 2.) LF-DRGS resulted in a delayed wash-out effect. All together these findings suggest that LF- $(1 \mathrm{~Hz})$ DRGS should be recommended for effective pain relief in PDPN. Moreover, the use of LF-DRGS will lead to a prolonged battery life as compared to MF- and HF-DRGS and thus has important consequences for costs-effectiveness of this treatment. 


\section{References}

1. Peltier A, Goutman SA, Callaghan BC. Painful diabetic neuropathy. BMJ 2014;348:g1799.

2. Tesfaye S, Boulton AJM, Dickenson AH. Mechanisms and management of diabetic painful distal symmetrical polyneuropathy. Diabetes Car 2013;36:2456-2465.

3. Waldfogel JM, Nesbit SA, Dy SM, Sharma R, Zhang A, Wilson LM et al. Pharmacotherapy for diabetic peripheral neuropathy pain and quality of life. Neurology 2017;90:1125.

4. Slangen R, Schaper NC, Faber CG, Joosten EA, Dirksen CD, Van Dongen RT et al. Spinal cord stimulation and pain relief in painful diabetic peripheral neuropathy: A prospective two-center randomized controlled trial. Diabetes Care 2014;37:3016-3024.

5. Van Beek M, Slangen R, Schaper NC, Faber CG, Joosten EA, Dirksen CD et al. Sustained treatment effect of spinal cord stimulation in painful diabetic peripheral Neuropathy: 24-Month Follow-up of a prospective Two-Center randomized controlled trial. Diabetes Care 2015;39:132-134.

6. De Vos CC, Meier K, Zaalberg PB, Nijhuis HJA, Duyvendak W, Vesper J et al. Spinal cord stimulation in patients with painful diabetic neuropathy: A multicentre randomized clinical trial. Pain 2014;155:24262431.

7. Deer TR, Grigsby E, Weiner RL, Wilcosky B, Kramer JM. A prospective study of dorsal root ganglion stimulation for the relief of chronic pain. Neuromodulation 2013;16:67-72.

8. Liem L, Russo M, Huygen FJPM, Van Buyten J-P, Smet I, Verrills P et al. A multicenter, prospective trial to assess the safety and performance of the spinal modulation dorsal root ganglion neurostimulator system in the treatment of chronic pain. Neuromodulation 2013;471-482.

9. Pope JE, Deer TR, Kramer J. A systematic review: current and future directions of dorsal root ganglion therapeutics to treat chronic pain. Pain Med 2013;14:1477-1496.

10. Eldabe S, Espinet A, Wahlstedt A, Kang P, Liem L, Patel NK et al. Retrospective case series on the treatment of painful diabetic peripheral neuropathy with dorsal root ganglion stimulation. Neuromodulation 2018;21:787-792.

11. Koetsier E, Franken G, Debets J, van Kuijk SMJ, Perez RSGM, Linderoth B et al. Effectiveness of dorsal root ganglion stimulation and dorsal column spinal cord stimulation in a model of experimental painful diabetic polyneuropathy. CNS Neurosci Ther 2018;25:367-374.

12. Calcutt NA. Modeling diabetic sensory neuropathy in rats. Methods Mol Med 2004;99:55-65.

13. Chaplan SR, Bach FW, Pogrel JW, Chung JM, Yaksh TL. Quantitative assessment of tactile allodynia in the rat paw. J Neurosci Methods 1994;53:55-63.

14. Beek M, Kleef $M$, Linderoth $B$, Kuijk SMJ, Honig WM, Joosten EA et al. Spinal cord stimulation in experimental chronic painful diabetic polyneuropathy: Delayed effect of High-frequency stimulation. Eur J Pain 2017;21:795-803.

15. Mills C, Leblond D, Joshi S, Zhu C, Hsieh G, Jacobson P et al. Estimating efficacy and drug ED50's using von frey thresholds: Impact of Weber's Law and log transformation. J Pain 2012;13:519-523.

16. Pluijms WA, Van Kleef M, Honig WM, Janssen SP, Joosten EA. The effect of spinal cord stimulation frequency in experimental painful diabetic polyneuropathy. Eur J Pain 2013;17:1338-1346.

17. Pan B, Yu H, Fischer GJ, Kramer JM, Hogan QH. Dorsal root ganglionic field stimulation relieves spontaneous and induced neuropathic pain in rats. J Pain 2016;17:1349-1358.

18. Wu M, Thorkilsen MM, Qin C, Farber JP, Linderoth B, Foreman RD. Effects of spinal cord stimulation on peripheral blood circulation in rats with streptozotocin-induced diabetes. Neuromodulation 2007;10:216223.

19. Gao J, Wu M, Li L, Qin C, Farber JP, Linderoth B et al. Effects of spinal cord stimulation with "standard clinical" and higher frequencies on peripheral blood flow in rats. Brain Res 2010;1313:53-61.

20. Kao CH, Chen JJJ, Hsu YM, Bau DT, Yao CH, Chen YS. High-frequency electrical stimulation can be a complementary therapy to promote nerve regeneration in diabetic rats. PLoS One 2013;8:e79078.

21. Deer TR, Levy RM, Kramer J, Poree L, Amirdelfan K, Grigsby E et al. Dorsal root ganglion stimulation yielded higher treatment success rate for CRPS and causalgia at 3 and 12 months. Pain 2016;158:669-681. 
22. Huygen F, Liem L, Cusack W, Kramer J. Stimulation of the L2-L3 Dorsal Root Ganglia Induces Effective Pain Relief in the Low Back. Pain Pract 2018;18:205-213.

23. Billet B, Wynendaele R, Vanquathem NE. Wireless Neuromodulation for Chronic Back Pain: Delivery of High-Frequency Dorsal Root Ganglion Stimulation by a Minimally Invasive Technique. Case Rep Med 2017;2017:1-4.

24. Billet B, Hanssens K, De Coster O, Nagels W, Weiner RL, Wynendaele R et al. Wireless high-frequency dorsal root ganglion stimulation for chronic low back pain: A pilot study. Acta Anaesthesiol Scand 2018; [Epub ahead of print]

25. Miller JP, Eldabe S, Buchser E, Johanek LM, Guan Y, Linderoth B. Parameters of Spinal Cord Stimulation and Their Role in Electrical Charge Delivery: A Review. Neuromodulation 2016;19:373-384.

26. Geurts JW, Joosten EA, Van Kleef M. Current status and future perspectives of spinal cord stimulation in treatment of chronic pain. Pain 2017;158, 771-774.

27. Koopmeiners AS, Mueller S, Kramer J, Hogan QH. Effect of electrical field stimulation on dorsal root ganglion neuronal function. Neuromodulation 2013;16:304-311; discussion 310-311.

28. Sandkühler J, Chen JG, Cheng G, Randić M. Low-frequency stimulation of afferent Adelta-fibers induces long-term depression at primary afferent synapses with substantia gelatinosa neurons in the rat. J Neurosci 1997;17:6483-6491.

29. Melzack R, Wall P. Pain mechanisms: a new theory. Science 1965;150:971-979.

30. Stiller CO, Cui JG, O'Connor WT, Brodin E, Meyerson BA, Linderoth B. Release of $\mathrm{Y}$-aminobutyric acid in the dorsal horn and suppression of tactile allodynia by spinal cord stimulation in mononeuropathic rats. Neurosurgery.1996;39:367-375.

31. Cui JG, O'Connor WT, Ungerstedt U, Linderoth B, Meyerson BA. Spinal cord stimulation attenuates augmented dorsal horn release of excitatory amino acids in mononeuropathy via a GABAergic mechanism. Pain 1997;73:87-95.

32. Linderoth B, Stiller CO, Gunasekera L, O'Connor WT, Ungerstedt U, Brodin E. Gamma-aminobutyric acid is released in the dorsal horn by electrical spinal cord stimulation: an in vivo microdialysis study in the rat. Neurosurgery 1994;34:484-488; discussion 488-489.

33. Linderoth B, Foreman RD. Conventional and Novel Spinal Stimulation Algorithms: Hypothetical Mechanisms of Action and Comments on Outcomes. Neuromodulation 2017;20:525-533.

34. Cui JG, Linderoth B, Meyerson BA. Effects of spinal cord stimulation on touch-evoked allodynia involve GABAergic mechanisms. An experimental study in the mononeuropathic rat. Pain. 1996;66:287-295.

35. Janssen SP, Gerard S, Raijmakers ME, Truin M, Van Kleef M, Joosten EA. Decreased intracellular GABA levels contribute to spinal cord stimulation-induced analgesia in rats suffering from painful peripheral neuropathy: The role of KCC2 and GABAAreceptor-mediated inhibition. Neurochem Int 2012;60:21-30.

36. Krames ES. The dorsal root ganglion in chronic pain and as a target for neuromodulation: A review. Neuromodulation. 2015;18:24-32.

37. Pawela CP, Kramer JM, Hogan QH. Dorsal root ganglion stimulation attenuates the BOLD signal response to noxious sensory input in specific brain regions: Insights into a possible mechanism for analgesia. Neuroimage 2017;147:10-18;

38. van Beek M, Hermes D, Honig WM, Linderoth B, Van Kuijk SMJ, Van Kleef M et al. Long-Term Spinal Cord Stimulation Alleviates Mechanical Hypersensitivity and Increases Peripheral Cutaneous Blood Perfusion in Experimental Painful Diabetic Polyneuropathy. Neuromodulation 2018;21:472-479.

39. Franken G, Debets J, Joosten EA. Dorsal Root Ganglion Stimulation in Experimental Painful Diabetic Peripheral Neuropathy: Burst vs. Conventional Stimulation Paradigm. Neuromodulation 2018; [Epub ahead of print] 



\section{Chapter}

\section{Mechanism of Dorsal Root Ganglion Stimulation for Pain Relief in Painful Diabetic Polyneuropathy is Not Dependent on GABA Release in the Dorsal Horn of the Spinal Cord}

Eva Koetsier, MD, ${ }^{1}$ Glenn Franken, MSc, ${ }^{2,3}$ Jacques Debets, ${ }^{4}$ Lonne Heijmans, ${ }^{3}$ Sander M.J. van Kuijk PhD, ${ }^{5}$ Bengt Linderoth, PhD, MD, ${ }^{6}$ Elbert A. Joosten, PhD, ${ }^{2,3}$ Paolo Maino, MD ${ }^{1}$

${ }^{1}$ Pain Management Center, Neurocenter of Southern Switzerland, Regional Hospital of Lugano, Via Capelli, 6962, Lugano, Switzerland

${ }^{2}$ Department of Anesthesiology and Pain Management, Maastricht University Medical Center ${ }^{+}$, Maastricht, the Netherlands

${ }^{3}$ Department of Translational Neuroscience, School of Mental Health and Neuroscience (MHeNS), University of Maastricht, the Netherlands

${ }^{4}$ Muroidean Facility, School of Cardiovascular Diseases (CARIM), Maastricht, the Netherlands

${ }^{5}$ Department of Clinical Epidemiology and Medical Technology Assessment, Maastricht University Medical Center ${ }^{+}$, Maastricht, the Netherlands.

${ }^{6}$ Department of Clinical Neuroscience, Karolinska Institutet, SE-171 77 Stockholm, Sweden

CNS Neuroscience \& Therapeutics. 2020;26:136-143. 


\section{Abstract}

Aims: It is hypothesized that dorsal root ganglion stimulation (DRGS), sharing some of the mechanisms of traditional spinal cord stimulation (SCS) of the dorsal columns, induces $\gamma$ aminobutyric acid (GABA)-release from interneurons in the spinal dorsal horn.

Methods: We used quantitative immunohistochemical analysis in order to investigate the effect of DRGS on intensity of intracellular GABA-staining levels in the L4-L6 spinal dorsal horn of painful diabetic polyneuropathy (PDPN) animals. To establish the maximal pain relieving effect, we tested for mechanical hypersensitivity to von Frey filaments and animals received 30 minutes of DRGS at day 3 after implantation of the electrode. One day later, 4 Sham-DRGS animals and 4 responders-to-DRGS received again 30 minutes of DRGS and were perfused at the peak of DRGS-induced pain relief.

Results: No significant difference in GABA-immunoreactivity was observed between DRGS and Sham-DRGS in lamina 1-3 of the spinal levels L4-6 neither ipsilaterally, nor contralaterally.

Conclusions: DRGS does not induce GABA release from the spinal dorsal horn cells, suggesting that the mechanisms underlying DRGS in pain relief are different from those of conventional SCS. The modulation of a GABA mediated "Gate Control" in the DRG itself, functioning as a prime Gate of nociception, is suggested and discussed. 


\section{Introduction}

In the western world, the prevalence of chronic pain is $30 \%^{1}$, and about one fifth of these persons has predominantly neuropathic pain. ${ }^{2-4}$ Neuropathic pain is described as pain caused by a lesion or a disease of the somatosensory nervous system. ${ }^{5}$ One of the most common neuropathic pain conditions is diabetic polyneuropathy (DPN), which is most typically a chronic, symmetrical, length-dependent sensory motor polyneuropathy. ${ }^{6} \mathrm{Up}$ to $1 / 3$ of all diabetic patients acquire painful diabetic polyneuropathy (PDPN), ${ }^{7}$ and pharmacological pain therapy is often insufficient in these patients. ${ }^{8}$ Conventional Spinal Cord Stimulation of the dorsal columns (hereafter labelled SCS) has resulted in significant pain reduction in many intractable PDPN patients. ${ }^{9-11}$ However, in about $40 \%$ of these patients SCS-treatment is not effective. ${ }^{9-11}$ Conventional Dorsal Root Ganglion Stimulation (hereafter named DRGS) acts at the level of the dorsal root ganglion (DRG), a promising new location for neuromodulation in managing selected pain conditions among those also PDPN. ${ }^{12}$ DRGS enables the physician to target a more peripheral, selective communication station for all nociceptive signalling from the peripheral nervous system to the dorsal horn in the spinal cord, from where the information is further processed via the spinothalamic tracts to the brain. ${ }^{13}$ The DRG plays an important role in both neuropathic and nociceptive pain conditions. ${ }^{14}$ Therefore, while SCS can theoretically only modulate $A \beta$ fibre signalling, DRGS might also be able to modulate A $\delta$ and C-type fibre signalling. ${ }^{15}$

A key molecule in the processing and modulation of the nociceptive signal in the spinal dorsal horn is $\gamma$-aminobutyric acid (GABA). ${ }^{16,17}$ Experimental data demonstrated decreased extracellular GABA levels and increased intracellular GABA levels in the dorsal horn after peripheral nerve injury. ${ }^{18,19} \mathrm{GABA}$ has been proposed to have a major role in nociceptive and non-nociceptive processing according to the Gate Control Theory. ${ }^{20}$ Based on this, it was suggested to stimulate non-nociceptive $A \beta$ fibres in the dorsal columns (SCS) aiming to turn on the Gate mechanisms and modulate the nociceptive input. In an experimental study of allodynic rats, increased levels of extracellular GABA were indeed found in the dorsal horns in response to SCS. ${ }^{18,21,22}$ The pivotal role of GABA in the analgesic effect of SCS has furthermore been confirmed by the fact that the application of the GABA 'responders-to-SCS'.23-26 The results of an experimental study of Janssen et al. ${ }^{27}$ demonstrated additionally that responders-to-SCS showed decreased levels of intracellular GABA-immunoreactivity (GABA-IR) in the spinal dorsal horn in comparison to non-responders-to-SCS and Sham-SCS animals. A relation between the release of intracellular accumulated GABA in the spinal cord dorsal horn and the analgesic effect of SCS was therefore hypothesized. ${ }^{27-30}$

The mechanisms underlying DRGS and its ensuing pain relief are as yet unknown, and it is likely that it shares some spinal and supraspinal mechanisms with SCS, dependent on $A \beta$ fibres activated by both types of stimulation. The present study is therefore aimed to 
investigate the hypothesis that DRGS induces GABA release from spinal dorsal horn cells. We used quantitative immunohistochemical analysis in an animal model of PDPN to study the effect of one single DRGS paradigm on the levels of spinal dorsal horn intracellular GABA.

\section{Methods}

\section{Ethical Statement}

The protocols for this study were approved by the Animal Care Committee of the Maastricht University Medical Centre (DEC 2013-079). The procedures were conducted in accordance with the guidelines of the European Directive for the Protection of Vertebrate Animals Used for Experimental and Other Scientific Purposes (86/609/EU).

\section{Animals}

The study was performed on 48 adult female Sprague-Dawley rats, weighing 170-230 g and 8 weeks old at the start of the experiments (Charles River, Maastricht, the Netherlands).They were preoperatively housed in pairs and postoperatively individually, in transparent plastic cages situated in a climate controlled room under a 12-h light/dark cycle with food and water ad libitium. All efforts were made to minimize the number of animals used and their suffering, and alternatives to in vivo techniques were considered.

\section{Induction of Diabetes Mellitus}

All animals fasted overnight before the induction of diabetes. Streptozotocin (STZ, SigmaAldrich, Schnelldorf, Germany) was freshly dissolved in sterile $\mathrm{NaCl} 0.9 \%$ to a solution of $65 \mathrm{mg} / \mathrm{mL}$. Animals were intraperitoneally injected with the STZ solution (65 mg/ $\mathrm{kg}$ ) to induce diabetes mellitus (DM). On day 4 post-STZ injection, blood glucose levels were measured from the saphenous vein, using an Accu-Chek Aviva ${ }^{\circledR}$ glucometer (Roche Diagnostics $\mathrm{GmbH}$, Mannheim, Germany). Rats developing diabetes, defined as a blood glucose level of $\geq 15 \mathrm{mmol} / \mathrm{L},{ }^{31}$ were included in the study.

\section{Development and Assessment of Mechanical Hypersensitivity}

Pain behaviour was assessed by testing mechanical hypersensitivity based on the hind limb paw withdrawal response to Von Frey filaments (bending forces 0.6, 1.2, 2.0, 3.6, $5.5,8.5,15.1$, and $28.84 \mathrm{~g}$ ). Rats were placed in individual cages with a wire mesh floor and were allowed to acclimate to the experimental set-up for 15 minutes. The $50 \%$ withdrawal threshold (WT) was determined by use of the up-down method, ${ }^{32}$ as previously described. ${ }^{33}$ The cut-off value was defined as the absence of the paw withdrawal response to a $28.84 \mathrm{~g}$ force to prevent tissue damage. The registered $50 \%$ 
WT's (measured in grams) were then multiplied by 10.000 and logarithmically transformed to conform with Weber's law ${ }^{34}$ and in order to obtain a linear scale.

Mechanical hypersensitivity was tested at baseline (pre-STZ injection), and weekly during 4 weeks post-STZ injection. Mechanical hypersensitivity, caused by PDPN, was defined to be present in case of a decrease of $\geq 0.2$ unit of the $\log ^{10}(10.000 \times 50 \%$ WT) when compared to baseline. Only animals that developed mechanical hypersensitivity were selected for this study and treated with DRGS.

\section{Implantation of DRGS Electrode}

Under general anaesthesia, bipolar electrodes were implanted unilaterally at the L5 DRG, according to the earlier described procedure (adapted from Pan et al.). ${ }^{35,36}$ In short, via a paravertebral incision the intervertebral foramen was exposed, the foramen was opened and the anode and cathode were implanted at the DRG. The electrode was then secured into the transverse process followed by closure of the incision in layers.

\section{DRGS}

For comparison with previous experimental studies regarding neurostimulation in PDPN animals, we used time and design as in previous (published) experiments. ${ }^{33,36}$ Animals were stimulated for 30 minutes at day 3 and 4 following implantation. The stimulation on day 3 was meant to establish the maximal pain relieving effect of DRGS, ${ }^{36}$ while the stimulation on day 4 was performed to activate the mechanisms of action before perfusing the animals at the 'peak of DRGS induced pain relief'. For stimulation the implanted electrode was connected to the pulse generator (A-M systems MultiStim Model 3800, fitted with an A-M systems 3820 stimulus isolator (A-M systems, Sequim, WA, USA)). To assess motor thresholds (MT), the current amplitude was increased during stimulation at $2 \mathrm{~Hz}$ with a pulse width of $0.2 \mathrm{msec}$, defining MT as the current at which any further increase resulted in hind limb movement. Stimulation was delivered at a current set at $66.7 \%$ of MT (around $0.18 \mathrm{~mA}$ ), a frequency of $50 \mathrm{~Hz}$ and a pulse width of $0.2 \mathrm{msec}$. Amplitude was set at zero for sham stimulation.

On the third day following implantation, mechanical hypersensitivity was assessed pre-DRGS, during stimulation at $\mathrm{t}=15$ minutes and $\mathrm{t}=30$ minutes, and 30 minutes postDRGS ( $t=60$ minutes). On the 4 th day after implantation, rats received again 30 minutes of DRGS, just before being perfused (at the peak of the DRGS induced pain relief). An animal was defined as a 'responder-to-DRGS' if it developed an increase in the $\log ^{10}$ $\left(10.000 \times 50 \%\right.$ WT) of $\geq 0.2$ when compared to the pre-DRGS baseline. ${ }^{36}$ 


\section{Tissue Preparation}

At the peak of DRGS induced pain relief (after 30 minutes of stimulation), 4 responderto-DRGS animals and 4 Sham-DRGS animals were anesthetized with pentobarbital (100 $\mathrm{mg} / \mathrm{kg}$ ) and perfused transcardially with $15 \%$ picric acid and $4 \%$ paraformaldehyde in 0.2 $\mathrm{M}$ phosphate buffer saline (PBS; $\mathrm{pH}$ 7.6). A laminectomy was performed to extract spinal cord $\mathrm{L} 4-\mathrm{L} 6$ regions, which were post-fixated overnight (at $4^{\circ} \mathrm{C}$ ) and cryoprotected in $10 \%$ sucrose for $24 \mathrm{~h}$, and then incubated for $72 \mathrm{~h}$ in $25 \%$ sucrose in $0.1 \mathrm{M} \mathrm{PBS}\left(\mathrm{pH} 7.6\right.$, at $\left.4^{\circ} \mathrm{C}\right)$. Thereafter, tissues were frozen using solid carbon dioxide. Transverse cryosections (30 $\mu \mathrm{m}$ thick) were mounted on gelatine coated glass slides and stored at $-20^{\circ} \mathrm{C}$ until staining. The cryosectioning was performed in a blinded fashion for treatment.

\section{Immunohistochemical Detection of GABA}

Slides were allowed to dry at room temperature for $2 \mathrm{~h}$ before being washed with Trisbuffered saline (TBS, $0.1 \mathrm{M}, \mathrm{pH} 7.6$ ), including 0.3\% Triton X-100 (TBS-T), TBS and TBS-T. Thereafter, the slides were blocked for anti-GABA immunohistochemistry by the incubation in 2\% normal donkey serum (Sigma-Aldrich, Zwijndrecht, the Netherlands, D9663) for $1 \mathrm{~h}$, diluted in TBS-T, and then incubated with rabbit anti-GABA polyclonal antibody (1:5000 diluted in TBS-T; Sigma- Aldrich, Zwijndrecht, the Netherlands, A2052) for $48 \mathrm{~h}$. After rinsing unbound primary antibody with TBS, sections were incubated for 2 $h$ with the secondary antibody alexa fluor 488 donkey anti-rabbit IgG (1:100 diluted in TBS-T; Invitrogen, Breda, the Netherlands, A21206). Lastly, slides were rinsed with TBS and coverslipped with TBS/glycerol (20\%/80\%).

\section{Immunoreactivity Analysis}

After the GABA-staining protocol was completed, sections were observed under an Olympus AX-70 microscope and immunohistochemical analysis was performed, as previously described by Janssen et al. (2012). ${ }^{27}$ Firstly, photomicrographs were taken from both ipsilateral and contralateral dorsal horns for the spinal levels L4-L6 using the Provis AX70 fluorescent microscope (Olympus) with a U-CMAD-2 black and white camera (Olympus) with CellP imaging software. Images were merged using Adobe Photoshop and grayscale values were analysed (blinded for treatment) using AnalySIS software. Regions of interest for the analysis of GABA-IR were lamina of Rexed 1-3 of the dorsal horn. ${ }^{37}$ Grayscale values were calculated for these laminae. The outcomes should depict the intracellular GABA content in the dorsal horns.

\section{Statistical Analysis}

The assay and the data-analysis were performed in a blinded fashion in an identical mode as published before. ${ }^{27}$ Data are represented as mean \pm standard error of the mean (SEM). Statistical analysis of grayscale values was performed using GraphPad Prism software. A 
Wilcoxon signed-rank test was used for comparison of mechanical hypersensitivity preSTZ injection and pre-implantation. For comparisons of grayscale values between levels ( $L 4$ vs. L5 vs. L6), treatments (DRGS vs. Sham-DRGS) and left-right differences (ipsilateral vs. contralateral), a two-way analysis of variance (ANOVA) was used, followed by a Sidak's multiple comparisons test. A P value $<0.05$ was considered significant.

\section{Results}

\section{Description of Cohorts of Animals}

Forty-three of the 48 animals (90\%) that were injected with STZ developed DM (blood

glucose $\geq 15 \mathrm{mmol} / \mathrm{L}$ ). Twenty-two out of the 43 diabetic animals developed painful neuropathy $\left(51 \%, \geq 0.2\right.$ decrease in $\log ^{10}(10.000 \times 50 \%$ WT), and were implanted with a DRGS system. Two animals were excluded from the study because of high MT (MT>1mA), and two due to a connector breakage. Eleven animals were selected for treatment with DRGS and 7 for treatment with Sham-DRGS. ${ }^{36}$ Of these animals, 4 responders-to-DRGS animals and 4 Sham-DRGS animals were selected for the GABA-IR analyses.

\section{Development of PDPN and Effect of DRGS on PDPN}

In the DRGS group, the $\log ^{10}(10.000 \times 50 \%$ WT) dropped significantly from 5.059 pre-STZ injection to 4.376 pre-DRGS $(p<0.01)$. In the Sham-DRGS group, the $\log ^{10}(10.000 \times 50 \%$ WT) dropped also significantly from 5.041 pre-STZ injection to 4.416 pre-Sham-DRGS ( $p<$ $0.05) .{ }^{36}$ The mean $\log ^{10}(10.000 \times 50 \%$ WT) value of the animals that were selected for GABA-IR analyses $(n=8)$ decreased from $4.99 \pm 0.05$ pre-STZ injection to $4.50 \pm 0.06$ preimplantation ( 4 weeks post-STZ injection, $p<0.01$ ). Motor thresholds were tested prior to DRGS (to select the appropriate amplitude). Normal responses were observed indicating no effect on motor behavior. We described the efficacy of DRGS in an earlier publicized study. ${ }^{36}$ In short, DRGS induced a complete reversal of mechanical hypersensitivity during stimulation. A return to pre-DRGS values was noted after cessation of DRGS at $t=60$ minutes. Sham-DRGS did not induce a reversal of mechanical hypersensitivity. Eight out of the 11 DRGS animals responded to DRGS (73\%) at $t=15$ minutes and 10 out of 11 (91\%) at $t=30$ minutes. All 4 DRGS animals that were selected for the GABA-IR analyses of the current study, were responders-to-DRGS. At 15 minutes of DRGS, 2/4 animals (50\%) responded to DRGS, while at 30 minutes 4/4 animals (100\%) responded. At 60 minutes (30 minutes after cessation of DRGS), 1/4 animals (25\%) still displayed a slight effect of the DRGS. 


\section{Chapter 6}

\section{Comparison of Mean Gray Values L4-L6}

The anti-GABA immunohistochemical analysis showed a strong GABA-IR, prevalently in laminae 1-3 of the spinal dorsal horn (Fig. 1, the contralateral staining results are not shown). No differences were found between ipsilateral and contralateral GABA-IR for both the DRGS group as well as the Sham-DRGS group in lamina 1-3 on spinal level L4-L6 (Fig. 2). Additionally, no differences in GABA-IR were found between DRGS and ShamDRGS in lamina 1-3 of spinal level L4-6, neither ipsilaterally nor contralaterally (Fig. 2).
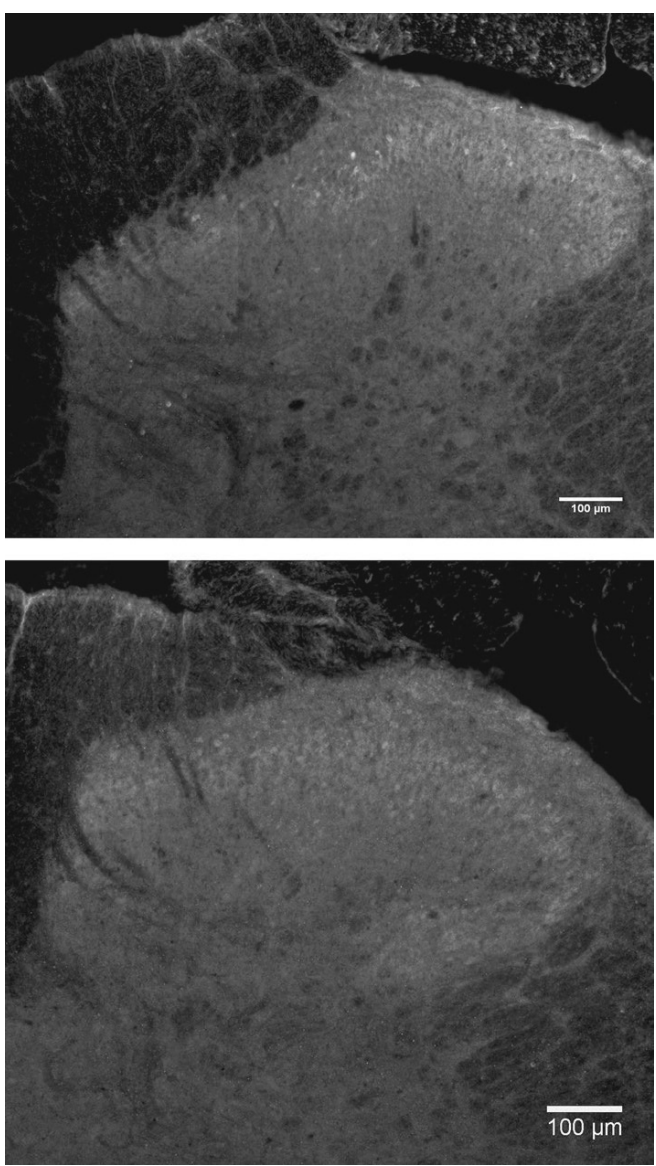

Figure 1. Representive images of the immunohistochemical staining of the upper laminae of the DH in animals implanted with DRGS electrode. No differences in GABA-IR were observed for the Sham-DRGS group (A) and DRGS group (B) in the upper laminae of the L4-L6 spinal segments. Additionally, no differences in GABA-IR were observed between the ipsilateral and contralateral DH in both the Sham-DRGS and DRGS group.

Scale bar $=100 \mu \mathrm{m}$.

GABA, $y$-aminobutyric acid; DRGS, Dorsal Root Ganglion Stimulation; DH, Dorsal Horn; GABA-IR, GABAimmunoreactivity. 


\section{GABA-immunoreactivity L4-6}

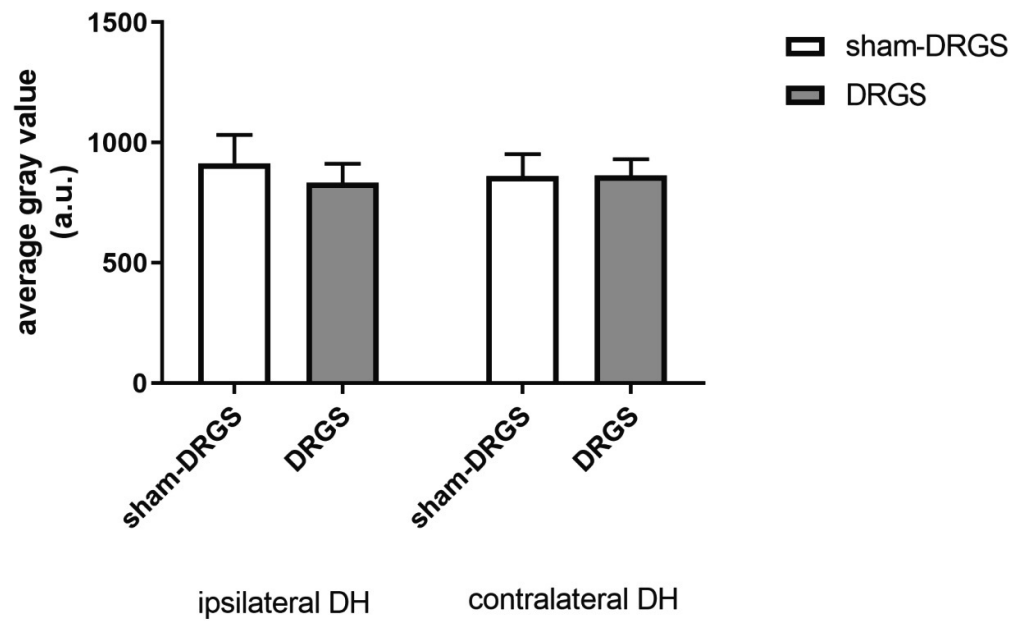

Figure 2. Average gray values of both the ipsilateral and contralateral dorsal horn in lamina 1-3 of spinal level L4-L6. Data are expressed as means \pm SEM.

GABA, y-aminobutyric acid; DRGS, Dorsal Root Ganglion Stimulation; DH, Dorsal Horn.

\section{Comparison of Mean Gray Values per Level}

No differences in terms of ipsilateral GABA-IR were observed between DRGS and ShamDRGS on all analysed levels ( $L 4: p>0.99 ; L 5: p>0.99, L 6: p=0.77$ ). Similarly, no differences were found in terms of ipsilateral GABA-IR for both DRGS ( $L 4$ vs. L5 $p=0.64$; L4 vs. L6 $p=0.98$; $L 5$ vs. $L 6 p=0.78$ ) and Sham-DRGS ( $L 4$ vs. $L 5 p=0.60 ; ~ L 4$ vs. $L 6 p=0.58 ; L 5$ vs. $L 6$ $p>0.99$ ) between each analysed level (Fig. 3).

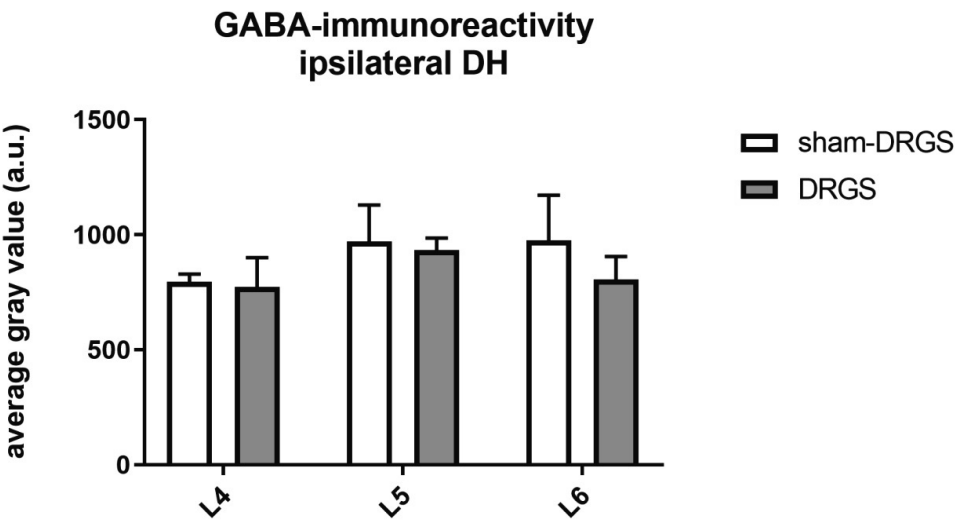

Figure 3. Average gray values of the ipsilateral dorsal horn in lamina 1-3 per spinal level (L4, L5 and L6). Data are expressed as means \pm SEM.

GABA, $\gamma$-aminobutyric acid; DH, Dorsal Horn; DRGS, Dorsal Root Ganglion Stimulation. 
No differences in terms of contralateral GABA-IR were observed between DRGS and Sham-DRGS on all analysed levels ( $L 4: p>0.99$; $L 5: p=0.57$, L6: $p=0.58$ ). Similarly, no differences were found in terms of contralateral GABA-IR for both DRGS (L4 vs. L5 $p=0.13$; L4 vs. L6 $p=0.99$; L5 vs. L6 p=0.25) and Sham-DRGS (L4 vs. L5 p=0.60; L4 vs. L6 p=0.27; L5 vs. L6 p>0.92) between each analysed level (Fig. 4).

\section{GABA-immunoreactivity contralateral DH}

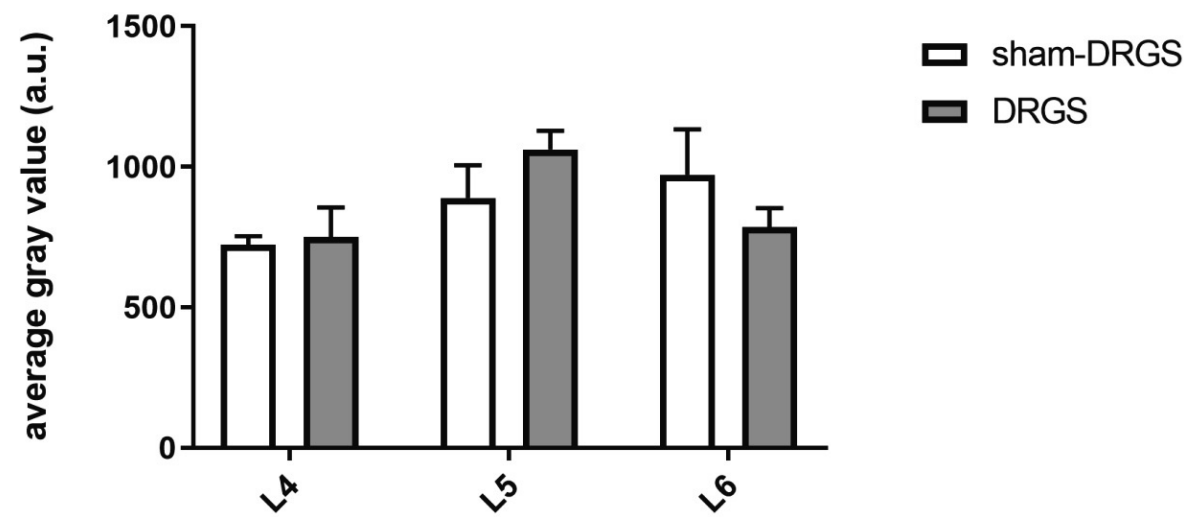

Figure 4. Average gray values of the contralateral dorsal horn in lamina 1-3 per spinal level (L4, L5 and L6). Data are expressed as means \pm SEM.

GABA, $y$-aminobutyric acid; DH, Dorsal Horn; DRGS, Dorsal Root Ganglion Stimulation.

\section{Discussion}

This is the first experimental study to assess intracellular GABA levels in the spinal dorsal horn at the peak of the DRGS pain relieving effect in a neuropathic pain model of PDPN. The results of this study indicate that DRGS does not result in decreased levels of intracellular GABA-IR in the spinal dorsal horn and thus does not induce GABA release. Dorsal column SCS has been demonstrated to induce GABA release from the spinal dorsal horn cells in agreement with the Gate Control Theory, ${ }^{18,27}$ while our results instead point to a different mechanism involved in DRGS and its production of pain relief. The assay and data-analysis utilized during our study were performed in an identical way as the previous study regarding GABA release in $\mathrm{SCS}^{27}$ with the difference that the previous study did not concern PDPN animals but neuropathic pain animals with a partial sciatic nerve ligation (according to the Seltzer model). From these data it was concluded that the assay used for this study is sensitive enough to detect changes in GABA-IR in the spinal dorsal horn. 
The dorsal horn is an essential and second order relay station for the integration and modulation of pain. ${ }^{16}$ The Gate Control Theory includes the pivotal role of inhibitory GABA-ergic interneurons modulating the nociceptive afferents and with this acts as the major regulatory component of the mechanism underlying SCS of dorsal columns. ${ }^{16,18-}$ $20,27,38$ Electrical stimulation of the ascending branch of the non-nociceptive large $A \beta$ fibres located in the dorsal column induces antidromic activation and generates synaptic interactions with GABA-ergic interneurons that inhibit the transmission of nociceptive signals entering the spinal dorsal horn via the slow-conducting $C$ fibers. ${ }^{16,38}$ The GABAergic inhibitory interneurons in the superficial laminae of the dorsal horn can be activated by $A \beta$-fibre inputs, which results in enhanced GABA release in the dorsal horn and an increase of extracellular GABA (Janssen et al., 2012). ${ }^{27}$ Peripheral nerve injury is known to induce a dysfunction of the natural GABA-ergic inhibition and a neuronal hyperexcitability in the spinal dorsal horn, which are among the major underlying causes of neuropathic pain. ${ }^{18,30,39-41}$ The theory that nerve injury induces a loss in GABA-ergic inhibition in the spinal dorsal horn, causing neuropathic pain, is sustained by experimental studies that showed that pharmacological antagonism of GABA-ergic inhibition either via the $G A B A_{A}$, but mainly via the $G A B A_{B}$ receptor in the spinal cord induced mechanical hypersensivity. ${ }^{42-44}$ Prior experimental studies confirm that SCS reduces the neuronal excitability and spinal pain transmission. ${ }^{16}$ The role of GABA was furthermore substantiated by experimental and clinical studies demonstrating that the intrathecal application of GABA-в receptor agonists like baclofen further potentiate the analgesic effects of SCS, ${ }^{24-26}$ while at the same time local perfusion of GABA-в receptor antagonists, like bicuculline, abolish this effect. ${ }^{21}$ Therefore, analgesic effects of SCS may be especially attributed to the activation of the GABA-B receptor. ${ }^{21}$ An experimental study of Janssen et al., ${ }^{27}$ demonstrated additionally a decrease of intracellular dorsal horn GABA-IR in responders-to-SCS, confirming a relation between the release of intracellular accumulated GABA and the analgesic effect of SCS.

Conventional SCS is not successful in all patients and pain relieving effects can decline over the years. ${ }^{9,11}$ Lack of anatomic specificity of the painful area and positional variations in stimulation are furthermore well known drawbacks of conventional SCS therapy. The DRG appears to be an appealing site for neurostimulation, ${ }^{45}$ and clinical evidence indicates that DRGS provides efficacious pain relief in neuropathic pain sufferers, ${ }^{46-50}$ which is confirmed by experimental studies. ${ }^{35,36}$ In comparison to SCS, DRGS has been demonstrated to have a better anatomic specificity of the painful area. ${ }^{50}$ Furthermore, with DRGS the electrical fields have a direct effect on the neural tissues that are pathophysiologically involved in the chronic pain disease condition. Since the location of stimulation is completely different with DRGS (local stimulation at the DRG) as compared to SCS (stimulation of the dorsal column) the question remains if both stimulation paradigms act via the same mechanism. Our quantitative immunohistochemical data indicate that, even though DRGS and SCS resulted in a similar decrease of PDPN, ${ }^{36}$ DRGS does not act via the stimulation-induced GABA mediated mechanisms in the dorsal horn. 
Clearly, DRGS does probably not only directly act via modulation of A $\beta$-fibres, but also of $A \delta$ - and C-type fibers. ${ }^{15}$ As $A \beta$-fibres are stimulated, one might expect that the analgesic effect of DRGS, like that of $\mathrm{SCS},{ }^{27}$ is also linked to activation of intracellular GABA release in the dorsal horn. However, the results of the current study suggest that the analgesic effect of DRGS is not linked to GABA release in the dorsal horn. A hypothetical explanation for this could be that the modulation of a GABA mediated Gate Control mechanism with DRGS actually takes place in a primary Gate for nociceptive control, namely at the DRG itself, instead of in the dorsal horn. This hypothesis is supported by a recently published experimental study of Du at al., ${ }^{51}$ which confirmed that key components of the GABA-ergic transmission are expressed in the DRG. Their study showed that depolarizing stimuli induce GABA release in the DRG. Additionally, their study demonstrated a reduction of the neuronal excitability in the DRG in response to GABA. Furthermore, focal infusion of GABA or GABA reuptake inhibitors into the DRG alleviated neuropathic pain, and the delivery of $G_{A B A}$ receptor antagonists to the DRG on the other hand exacerbated peripherally induced nociception. ${ }^{51}$ These results indicate that there is a endogenous GABA-ergic control in the DRG, ${ }^{52,53}$ and analgesic effects of focally applied GABA mimetics suggest that DRGS acts via the modulation of a GABA mediated Gate Control at the level of the DRG.

In conclusion, DRGS does not induce GABA release in spinal dorsal horn of neuropathic (PDPN) rats. With this observation we suggest that the mechanism underlying DRGS-induced pain relief is different from that of dorsal column SCS. Further research is warranted to elucidate the mechanism underlying DRGS in pain relief. The modulation of a GABA mediated "Gate Control" in the DRG, functioning as a prime Gate of nociception, is suggested. 


\section{References}

1. Leadley RM, Armstrong N, Lee YC, Allen A, Kleijnen J. Chronic Diseases in the European Union: The Prevalence and Health Cost Implications of Chronic Pain. J Pain Palliat Care Pharmacother. 2012; 26:310325.

2. Cohen SP, Mao J. Neuropathic pain: mechanisms and their clinical implications. BMJ. 2014;348:f7656.

3. Torrance N, Smith BH, Bennett MI, Lee AJ. The Epidemiology of Chronic Pain of Predominantly Neuropathic Origin. Results From a General Population Survey. J Pain. 2006;7:281-289.

4. Bouhassira D, Lantéri-Minet M, Attal N, Laurent B, Touboul C. Prevalence of chronic pain with neuropathic characteristics in the general population. Pain. 2008;136:380-387.

5. Treede RD, Jensen TS, Campbell JN, Cruccu G, Dostrovsky JO, Griffin JW, et al. Neuropathic pain: Redefinition and a grading system for clinical and research purposes. Neurology. 2008;70:1630-1635.

6. Tesfaye S, Boulton AJ, Dyck PJ, Freeman R, Horowitz M, Kempler P, et al. Diabetic neuropathies: Update on definitions, diagnostic criteria, estimation of severity, and treatments. In: Diabetes Care. 2010;33:22852293.

7. Abbott CA, Malik RA, Van Ross ER, Kulkarni J, Boulton AJM. Prevalence and characteristics of painful diabetic neuropathy in a large community-based diabetic population in the U.K. Diabetes Care. 2011;34:2220-2224.

8. Tesfaye S, Boulton AJM, Dickenson AH. Mechanisms and management of diabetic painful distal symmetrical polyneuropathy. Diabetes Care. 2013;36:2456-2465.

9. Slangen R, Schaper NC, Faber CG, Joosten EA, Dirksen CD, van Dongen RT, et al. Spinal cord stimulation and pain relief in painful diabetic peripheral neuropathy: A prospective two-center randomized controlled trial. Diabetes Care. 2014;37:3016-3024.

10. van Beek M, Slangen R, Schaper NC, Faber CG, Joosten EA, Dirksen CD, et al. Sustained treatment effect of spinal cord stimulation in painful diabetic peripheral Neuropathy: 24-Month Follow-up of a prospective Two-Center randomized controlled trial. Diabetes Care. 2015;38:e132-134.

11. de Vos CC, Meier K, Zaalberg PB, Nijhuis HJ, Duyvendak W, Vesper J, et al. Spinal cord stimulation in patients with painful diabetic neuropathy: A multicentre randomized clinical trial. Pain. 2014;155:24262431.

12. Eldabe S, Espinet A, Wahlstedt A, Kang P, Liem L, Patel NK, et al. Retrospective case series on the treatment of painful diabetic peripheral neuropathy with dorsal root ganglion stimulation. Neuromodulation. 2018;21:787-792.

13. Chapman KB, van Helmond N. Mechanisms of Dorsal Root Ganglion Stimulation in Pain Suppression: Time to Consider Alternative Mechanisms of Action? Neuromodulation. 2018;21:522-523.

14. Deer TR, Krames E, Mekhail N, Pope J, Leong M, Stanton-Hicks M, et al. The appropriate use of neurostimulation: New and evolving neurostimulation therapies and applicable treatment for chronic pain and selected disease states. Neuromodulation. 2014;17:599-615.

15. Koopmeiners AS, Mueller S, Kramer J, Hogan QH. Effect of electrical field stimulation on dorsal root ganglion neuronal function. Neuromodulation. 2013;16:304-311.

16. Guan Y. Spinal cord stimulation: Neurophysiological and neurochemical mechanisms of action. Curr Pain Headache Rep. 2012;16:217-225.

17. Guan Y, Wacnik PW, Yang F, Carteret AF, Chung CY, Meyer RA, et al. Spinal cord stimulation-induced analgesia: Electrical stimulation of dorsal column and dorsal roots attenuates dorsal horn neuronal excitability in neuropathic rats. Anesthesiology. 2010;113:1392-1405.

18. Stiller CO, Cui JG, O'Connor WT, Brodin E, Meyerson BA, Linderoth B. Release of $\mathrm{Y}$-aminobutyric acid in the dorsal horn and suppression of tactile allodynia by spinal cord stimulation in mononeuropathic rats. Neurosurgery. 1996;39:367-375.

19. Janssen SP, Truin M, Van Kleef M, Joosten EA. Differential GABAergic disinhibition during the development of painful peripheral neuropathy. Neuroscience. 2011;184:183-194.

20. Melzack R, Wall P. Pain mechanisms: a new theory. Science. 1965;150:971-979. 


\section{Chapter 6}

21. Cui JG, O'Connor WT, Ungerstedt U, Linderoth B, Meyerson BA. Spinal cord stimulation attenuates augmented dorsal horn release of excitatory amino acids in mononeuropathy via a GABAergic mechanism. Pain. 1997;73:87-95.

22. Linderoth B, Stiller CO, Gunasekera L, O'Connor WT, Ungerstedt U, Brodin E. Gamma-aminobutyric acid is released in the dorsal horn by electrical spinal cord stimulation: an in vivo microdialysis study in the rat. Neurosurgery. 1994;34:484-488.

23. Linderoth B, Foreman RD. Conventional and Novel Spinal Stimulation Algorithms: Hypothetical Mechanisms of Action and Comments on Outcomes. Neuromodulation. 2017;20:525-533.

24. Cui JG, Linderoth B, Meyerson BA. Effects of spinal cord stimulation on touch-evoked allodynia involve GABAergic mechanisms. An experimental study in the mononeuropathic rat. Pain. 1996;66:287-295.

25. Lind G, Meyerson BA, Winter J, Linderoth B. Intrathecal baclofen as adjuvant therapy to enhance the effect of spinal cord stimulation in neuropathic pain: A pilot study. Eur J Pain. 2004;8:377-383.

26. Lind G, Schechtmann G, Winter J, Meyerson BA, Linderoth B. Baclofen-enhanced spinal cord stimulation and intrathecal baclofen alone for neuropathic pain: Long-term outcome of a pilot study. Eur J PAIN. 2008;12:132-136.

27. Janssen SP, Gerard S, Raijmakers ME, Truin M, Van Kleef M, Joosten EA. Decreased intracellular GABA levels contribute to spinal cord stimulation-induced analgesia in rats suffering from painful peripheral neuropathy: The role of KCC2 and GABAAreceptor-mediated inhibition. Neurochem Int. 2012;60:21-30

28. Eaton MJ, Plunkett JA, Karmally S, Martinez MA, Montanez K. Changes in GAD- and GABAimmunoreactivity in the spinal dorsal horn after peripheral nerve injury and promotion of recovery by lumbar transplant of immortalized serotonergic precursors. J Chem Neuroanat. 1998;16:57-72. doi:10.1016/S0891-0618(98)00062-3

29. Kim J, Kim SJ, Lee H, Chang JW. Effective neuropathic pain relief through sciatic nerve administration of GAD65-expressing rAAV2. Biochem Biophys Res Commun. 2009;388(1):73-78. doi:10.1016/j.bbrc. 2009.07.120

30. Moore KA, Kohno T, Karchewski LA, Scholz J, Baba H, Woolf CJ. Partial peripheral nerve injury promotes a selective loss of GABAergic inhibition in the superficial dorsal horn of the spinal cord. J Neurosci. 2002;22:6724-6731.

31. Calcutt N a. Modeling diabetic sensory neuropathy in rats. Methods Mol Med. 2004;99:55-65.

32. Chaplan SR, Bach FW, Pogrel JW, Chung JM, Yaksh TL. Quantitative assessment of tactile allodynia in the rat paw. J Neurosci Methods. 1994;53:55-63.

33. Pluijms WA, Van Kleef M, Honig WM, Janssen SP, Joosten EA. The effect of spinal cord stimulation frequency in experimental painful diabetic polyneuropathy. Eur J Pain. 2013;17:1338-1346.

34. Mills C, Leblond D, Joshi S, et al. Estimating efficacy and drug ED50's using von frey thresholds: Impact of Weber's Law and log transformation. J Pain. 2012;13:519-523.

35. Pan B, Yu H, Fischer GJ, Kramer JM, Hogan QH. Dorsal Root Ganglionic Field Stimulation Relieves Spontaneous and Induced Neuropathic Pain in Rats. J Pain. 2016;17:1349-1358.

36. Koetsier E, Franken G, Debets J, van Kuijk SMJ, Perez RSGM, Linderoth B, et al. Effectiveness of dorsal root ganglion stimulation and dorsal column spinal cord stimulation in a model of experimental painful diabetic polyneuropathy. CNS Neuroscience and Therapeutics. 2019;25:367-374.

37. Magoul R, Onteniente B, Geffard M, Calas A. Anatomical distribution and ultrastructural organization of the gabaergic system in the rat spinal cord. An immunocytochemical study using anti-GABA antibodies. Neuroscience. 1987;20:1001-1009.

38. Smits $\mathrm{H}$, van Kleef $\mathrm{M}$, Holsheimer J, Joosten EA. Experimental spinal cord stimulation and neuropathic pain: mechanism of action, technical aspects, and effectiveness. Pain Pract. 2013;13:154-168.

39. Patel S, Naeem S, Kesingland A, Froestl W, Capogna M, Urban L, et al. The effects of GABA(B) agonists and gabapentin on mechanical hyperalgesia in models of neuropathic and inflammatory pain in the rat. Pain. 2001;90:217-226.

40. Somers DL, Clemente FR. Dorsal horn synaptosomal content of aspartate, glutamate, glycine and GABA are differentially altered following chronic constriction injury to the rat sciatic nerve. Neurosci Lett. 2002;323:171-174. 
41. Drew GM, Siddall PJ, Duggan AW. Mechanical allodynia following contusion injury of the rat spinal cord is associated with loss of GABAergic inhibition in the dorsal horn. Pain. 2004;109:379-388.

42. Hao JX, Xu XJ, Wiesenfeld-Hallin Z. Intrathecal $\gamma$-aminobutyric acidB (GABAB) receptor antagonist CGP 35348 induces hypersensitivity to mechanical stimuli in the rat. Neurosci Lett. 1994;182:299-302.

43. Malan TP, Mata HP, Porreca F. Spinal GABA(A) and GABA(B) receptor pharmacology in a rat model of neuropathic pain. Anesthesiology. 2002;96:1161-1167.

44. Sivilotti L, Woolf CJ. The contribution of GABAA and glycine receptors to central sensitization: disinhibition and touch-evoked allodynia in the spinal cord. J Neurophysiol. 1994;72:169-179.

45. Krames ES. The dorsal root ganglion in chronic pain and as a target for neuromodulation: A review. Neuromodulation. 2015;18:24-32.

46. van Buyten J-P, Smet I, Liem L, Russo M, Huygen F. Stimulation of Dorsal Root Ganglia for the Management of Complex Regional Pain Syndrome: A Prospective Case Series. Pain Pract. 2014;15:208-216.

47. Deer TR, Grigsby E, Weiner RL, Wilcosky B, Kramer JM. A prospective study of dorsal root ganglion stimulation for the relief of chronic pain. Neuromodulation. 2013;16:67-72.

48. Pope JE, Deer TR, Kramer J. A Systematic Review: Current and Future Directions of Dorsal Root Ganglion Therapeutics to Treat Chronic Pain. Pain Med. 2013;14:1477-1496.

49. Deer TR, Levy RM, Kramer J, Poree L, Amirdelfan K, Grigsby E, et al. Dorsal root ganglion stimulation yielded higher treatment success rate for CRPS and causalgia at 3 and 12 months. Pain. 2017;158:669-681.

50. Schu S, Gulve A, EIDabe S, Baranidharan G, Wolf K, Demmel W, et al. Spinal Cord Stimulation of the Dorsal Root Ganglion for Groin Pain-A Retrospective Review. Pain Pract. 2015;15:293-299.

51. Du X, Hao H, Yang Y, Huang S, Wang C, Gigout S, et al. Local GABAergic signaling within sensory ganglia controls peripheral nociceptive transmission. J Clin Invest. 2017;127:1741-1756.

52. Obradovic AL, Scarpa J, Osuru HP, Weaver JL, Park JY, Pathirathna S, et al. Silencing the $\alpha 2$ subunit of $\gamma$ aminobutyric acid type $A$ receptors in rat dorsal root ganglia reveals its major role in antinociception posttraumatic nerve injury. Anesthesiology. 2015;123:654-667.

53. Naik AK, Pathirathna S, Jevtovic-Todorovic V. GABAA receptor modulation in dorsal root ganglia in vivo affects chronic pain after nerve injury. Neuroscience. 2008;154:1539-1553. 



\section{Chapter 7}

\section{Summary and Discussion}




\section{Summary and Discussion}

The aim of the research as described in this thesis was to establish and improve the effectiveness of dorsal root ganglion stimulation (DRGS) for pain relief in painful polyneuropathy (see General Introduction Chapter 1, section 6.1, Aim of the Thesis). Additionally, an animal model is developed for DRGS in painful polyneuropathy which is aimed to further understand the mechanism of DRGS in pain relief in painful polyneuropathy and with that to increase effectiveness of this therapy.

The first research question (RQ 1) of this thesis is the question if DRGS results in pain relief in patients with painful polyneuropathy. This question is addressed and studied in Chapters $\mathbf{2}$ and $\mathbf{3}$. In Chapter $\mathbf{2}$ a 74-year-old male patient is presented, which suffered from severe intractable pain, dysesthesia and allodynia of the left foot caused by idiopathic small fiber neuropathy (SFN), confirmed by skin biopsy. The pain was not satisfactory controlled by pharmacological pain management. Hence, a DRGS lead was implanted at the left L5 DRG and connected to an external pulse generator (EPG). After a successful trial, a permanent implantable pulse generator (IPG) was implanted and significant pain reduction was noted for at least 20 months. The results from this case report suggested that the DRG is a promising target for neurostimulation to treat localized SFN. This resulted in a design and execution of a small-scale prospective pilot study on the effect of DRGS in painful polyneuropathy at our Pain Management Center at Lugano, which is presented and discussed in Chapter 3. Interestingly, during the conduction of this study, two small retrospective case series were published, one related to DRGS treatment for painful diabetic polyneuropathy (PDPN), ${ }^{1}$ and the other concerning DRGS treatment for hereditary and idiopathic polyneuropathy. ${ }^{2}$ Both studies showed a significant pain reduction with DRGS treatment, which remained stable during follow up of 12 and 6 months, respectively. ${ }^{1,2}$

The primary aim of the study presented in Chapter $\mathbf{3}$ was to analyze effectiveness of conventional DRGS (frequency $20 \mathrm{~Hz}$; pulse width 0.2-0.4 ms; amplitude $0.6 \mathrm{~mA}$ ) on pain relief in patients with intractable localized painful polyneuropathy in the lower limbs. Treatment was defined to be successful if there was a reduction of $\geq 50 \%$ in daytime and/or night-time pain intensity. Six out of the 7 implanted patients (85.7\%) showed stable treatment success during the follow up period of 6 months. This responder rate is significant increased as compared to the reported $60 \%$ responder rate in dorsal column SCS studies in patients with PDPN. ${ }^{3-5}$

The majority of literature examining SCS in the treatment of painful polyneuropathy relates to PDPN patients, ${ }^{3,5-13}$ while several case reports/series showed positive results of SCS and DRGS also in painful polyneuropathy of other etiologies than PDPN. ${ }^{14-16}$ An important aspect of our prospective study as compared to published clinical studies on effectiveness of SCS in PDPN is thus the fact that the etiology of polyneuropathy in our study was heterogeneous and not exclusively PDPN. In our study, the significant pain relieving effect in the subpopulation of the 2 PDPN patients was similar as compared to 
the 5 patients with polyneuropathy of other etiologies (idiopathic, chronic inflammatory demyelinating polyneuropathy, chemotherapy-induced peripheral neuropathy). This suggest that DRGS has the ability to not only successful manage pain in PDPN but also in other etiologies of painful polyneuropathy. This is even more important as the prevalence of other conditions leading to painful polyneuropathies is rising. ${ }^{17-19}$ Therefore, we feel that impact of our prospective study is significant as it provides data on effect of DRGS on painful polyneuropathy of other etiology and thus may induce larger scale randomized studies to confirm effectiveness, eventually in comparison to SCS.

The understanding of the spinal mechanisms of action underlying pain relief with SCS or DRGS is still incomplete. In neuropathic pain, multimodal wide-dynamic range (WDR) cells in the spinal dorsal horns express hyperexcitability, ${ }^{20}$ which seems to be related to an increased release of excitatory amino acids such as glutamate, and a dysfunction of the (natural) gamma-amino-butyric acid (GABA)ergic inhibition. ${ }^{21-24}$ According to the Gate Control Theory, electrical stimulation of the large myelinated non-nociceptive $A_{\beta}$ fibers in the spinal dorsal column induces the spinal inhibitory interneurons to release GABA, inactivating the WDR neurons, ${ }^{20}$ subsequently leading to an inhibition of the spinal nociceptive signal ("closing the gate") from the small nociceptive $A_{\delta}$ and C fibers to the brain (see General Introduction Chapter 1, section 4.1, Spinal Cord Stimulation and section 4.3.2, Analgesic Effect of SCS or DRGS: Mechanism of Action and a Role of GABA). ${ }^{20,24-27}$ Furthermore, experimental SCS has not only shown to inhibit dorsal horn WDR hyperexcitability and induce a release of GABA in the dorsal horn, but also to lead to a decrease of the interstitial glutamate concentration. ${ }^{20,22,24}$ Within the dorsal horn of the spinal cord, not only WDR projection neurons are involved in pain processing but also nociceptive specific (NS) projection neurons (Figure 1). ${ }^{28-30}$ While NS neurons respond to high-intensity input, WDR neurons are able to give long range responses to a much broader range of stimuli (therefore called 'wide dynamic range'). ${ }^{28}$

The Gate Control Theory only partially explains the mechanism of SCS-induced pain relief. It is for example not fully understood why SCS does not affect the perception of acute pain, as the Gate Control Theory predicts that activation of large myelinated fibers should mask all pain. ${ }^{29}$ The fact that SCS does not affect the perception of acute pain might be explained by the assumption that WDR and NS neurons may respond differently to SCS: NS neurons being active during noxious stimuli while unresponsive to SCS. ${ }^{29}$

Additionally, also supraspinal mechanisms have been shown to play an important role in the pain-relieving effect of SCS. ${ }^{24,31}$ SCS gives rise to orthodromic activation of a supraspinal network, finally resulting in descending inhibition of the dorsal horn nociceptive network. ${ }^{24}$ As a matter of fact, SCS has been shown to activate brainstem nuclei which are involved in the modulation of the incoming nociceptive signal in the spinal dorsal horn via descending serotonergic, noradrenergic, and dopaminergic fibers. $^{32-35}$

Not only brainstem nuclei are activated by SCS of the dorsal columns but also supraspinal cortical nuclei and areas, as has been demonstrated by imaging studies. 
Functional MRI studies have shown that SCS leads to decreased connectivity between somatosensory and limbic areas. ${ }^{36}$ This suggests that pain relief from SCS may include and reduce negative emotional processing associated with pain. Other functional MRI studies suggest the thalamus as potential mediator in the pain relieving mechanisms of sCS treatment. ${ }^{37,38}$

There is also evidence that SCS causes peripheral vasodilatation relieving ischemic pain and improving peripheral blood flow. ${ }^{39-42}$ Considering the vascular mechanisms that are involved in PDPN pathology, it is expected that improved blood perfusion of tissue leads to a better functioning of nervous tissue in PDPN patiens. ${ }^{42,43}$ Peripheral vasodilatation is mediated by antidromic release of calcitonin gene-related peptide (CGRP) through activation of predominantly small unmyelinated C-fibers, but also CGRP positive $A_{\delta}$ fibers (Figure 1). ${ }^{44,45}$ Other vasodilatory pathways should also be taken into account, such as a decreased sympathetic outflow during SCS. ${ }^{46-48}$ 


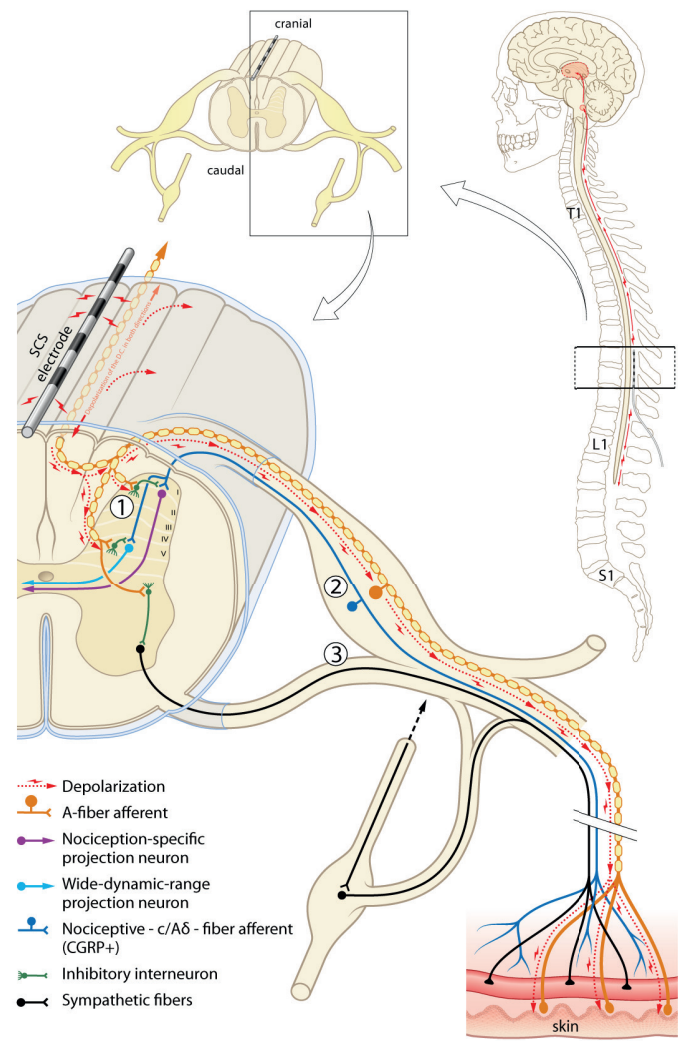

\section{Figure 1.}

Cartoon representing the anatomical basis for the known segmental working mechanism of dorsal column SCS in PDPN related to pain relief and vasodilation. Within the spinal dorsal horn there are two main types of projection neurons involved in pain processing: nociceptive specific (NS) located in the superficial laminae of the dorsal horn and wide dynamic range (WDR) neurons located in the deeper laminae of the dorsal horn. The projection neurons are located in lamina I (NS-neurons) and in laminae III-V (WDR) neurons and receive inputs from primary afferents (directly and via spinal interneurons), and from descending pathways (not shown). Among the primary afferents are large-diameter, myelinated non-nociceptive $A_{\beta}$ fibers, thinly myelinated nociceptive $A_{\delta}$ fibers, and small-diameter unmyelinated nociceptive C-fibers. The spinal nociceptive network also contains different excitatory (not shown) and GABA-ergic inhibitory interneurons, involved in "gating" sensory input to the brain ("Gate-Control theory"). Electrical stimulation of the dorsal columns results in antidromic impulses propagating along the large-diameter $A_{\beta}$ fibers activating the dorsal horn nociceptive network including the GABA-ergic inhibitory interneurons.

Neuromodulatory effects in the dorsal horn include suppression of WDR projection neurons via GABA release (1), antidromic activation of CGRP+ nociceptive afferents (2) and sympathetic inhibition (3). The release of CGRP from the free nerve endings and decrease in noradrenaline release from sympathetic fibers results in peripheral vasodilation.

SCS, Spinal cord stimulation; PDPN, painful diabetic polyneuropathy; NS, nociceptive specific; WDR, wide dynamic range; GABA, gamma-amino-butyric acid; CGRP, calcitonin gene-related peptide.

With permission from reference $\left[{ }^{43}\right]$ and artist impression by Rogier Trompert Medical-art Inc. 
The DRG is the first relay station in the nociceptive network and mediates nociception from the peripheral nerves to the central nervous system, ${ }^{49}$ and thus may form an appealing new location for neurostimulation. The DRG is of pivotal importance in the development and maintenance of chronic pain, as it exhibits pathophysiologic changes during chronic pain states, like altered electrophysiological membrane properties, changes in the expression of integral membrane proteins, and altered gene expression. ${ }^{50-}$ 52 Elevated excitability of sensory neurons in the DRG contributes to the pathogenesis of chronic pain that follows peripheral nerve injury, ${ }^{53}$ and treatment at this specific site is interesting as it may interact with this pathogenic processes.

Furthermore, the DRG represents the sensory gateway to the spinal cord, containing sensory neuron somata for all sensory modalities and fiber types: not only the nonnociceptive $A_{\beta}$ cell bodies but also the $A_{\delta}$ and $C$-type nociception related cell bodies (figure 1). While with SCS only the $A_{\beta}$ fibers in the dorsal columns are stimulated, DRGS very likely stimulates not only $A_{\beta}$ but also the $A_{\delta}$ or C-fibers. ${ }^{24,54}$ Additionally, it is expected that the vasodilating effect of SCS also occurs with the application of DRGS. As described above, a decreased sympathetic outflow during SCS is thought to be (at least partially) the cause of vasodilatation. Animal studies have shown sympathetic fibers to sprout into the DRG after peripheral nerve injury thereby forming abnormal connections with sensory neurons. ${ }^{55-57}$ It is reasonable to assume that this sympathetic fiber sprouting into the DRG also occurs in patients with painful polyneuropathy and therefore DRG might be a better target for neurostimulation.

Conventional SCS of the dorsal columns (frequency $20-80 \mathrm{~Hz}$; pulse width $0.2-0.5 \mathrm{~ms}$; amplitude 3.6 to $8.5 \mathrm{~mA})^{13,58}$ is often unable to cover difficult-to-reach areas like the feet and the groin without generating extensive paresthesias or motor side effects (see General Introduction Chapter 1, section 4.1, Spinal Cord Stimulation). ${ }^{59}$ DRGS, on the other hand, has been shown to cover these difficult-to-reach areas better (see General Introduction Chapter 1, section 4.2, Dorsal Root Ganglion Stimulation). ${ }^{59-62}$ As in painful polyneuropathy the pain is initially mainly located in the lower extremities (feet), ${ }^{63}$ we hypothesize that stimulation of lumbar DRG is more effective as compared to SCS and this is tested in relation to RQ2 in an animal model. With RQ2A we intended to develop an animal model for DRGS in PDPN and the results are presented in Chapter 4: DRGS bipolar leads were manufactured according to a procedure previously described by Pan et al. ${ }^{64}$ With various technical improvements and adapted surgical technique an animal model for DRGS in PDPN was established. This system was successfully tested in an already operational and meticulously tested PDPN animal model at the Maastricht University Medical Center+ with female Sprague-Dawley rats, ${ }^{42,65,66}$ and with this we established a reproducible animal model for DRGS in PDPN.

After responding positively to RQ2A, the answer to RQ2B could be assessed. RQ2B questioned whether DRGS is more effective in pain relief than SCS in experimental PDPN. In the study presented in Chapter 4, DM was induced in the animals with an intraperitoneal injection of $65 \mathrm{mg} / \mathrm{kg}$ streptozotocin (STZ) $(\mathrm{n}=48)$. Mechanical paw 
withdrawal thresholds were assessed with von Frey testing (see General Introduction Chapter 1, section 4.3, Stimulation Parameters, Mechanism of Action and Experimental Research). ${ }^{65}$ From the results of the study we conclude that effectiveness for pain relief with DRGS and SCS with conventional parameters (frequency $40-50 \mathrm{~Hz}$, pulse width 0.2 ms, amplitude 0.19) is similar in PDPN animals. However, we underscore that the experiments for this study were only based on short-term stimulation paradigms (30 minutes), which might be a limitation in the translation to the clinical situation where long-term stimulation protocols are used. Additionally, the concept underlying DRGS differs from SCS and therefore it is important to test also other stimulation settings like different frequencies and amplitude in an experimental model.

As the anatomical location of the DRG offers a closer proximity of the electrodes to the nociceptive and non-nociceptive fibers as with SCS of dorsal columns, reduced charge is required for similar electrical field. ${ }^{59,67-69}$ With SCS the presence of highly conductive cerebrospinal fluid (CSF) around the spinal cord can shunt electrical current away from the targeted region, ${ }^{70}$ while there is a scarcity of CSF around the DRG. ${ }^{71}$ Therefore, in clinical practice for DRGS lower amplitude (and charge) is required than for SCS, leading to longer battery life and therefore lower health care costs. Indeed, in clinical studies effective DRGS treatment is achieved at lower amplitudes, requiring on average about $15 \%$ of the power output of SCS. ${ }^{72}$ Still, in our animal study intensity of DRGS and SCS was similar (Chapter 4) (amplitude: $0.18 \pm 0.05 \mathrm{~mA}$ and $0.19 \pm 0.01 \mathrm{~mA}$, respectively). Both DRGS and SCS stimulation intensities were applied at $66.7 \%$ of motor threshold (MT). MT, defined as the lowest amplitude that induce contractions of the lower trunk or hind limbs, was thus determinative for amplitude high. The standardization of amplitude in the DRGS and SCS animals in our study enabled us to objectively compare results of DRGS and SCS with conservative settings.

A first attempt to study the effect of intensity of SCS and pain relief has been made by Meuwissen et al., ${ }^{73}$ who studied the effect of the intensity of conventional SCS (comparing 33, 50 and 66\% of $\mathrm{MT}$ ) in an experimental model of peripheral neuropathy. From this study it was concluded that the efficacy and pain relief of conventional SCS was linearly related to increasing amplitude.

Our model for DRGS in PDPN animals allowed future research on effectiveness of other clinically relevant stimulation paradigms and hence RQ3 was formulated questioning whether frequency is an important parameter in DRGS for pain relief in an animal model of PDPN. Novel advances in neurostimulation frequencies for SCS have emerged in the latest years, like high frequency SCS (with frequencies up to $10 \mathrm{kHz}$ ) and burst SCS $(40 \mathrm{~Hz}$ provided in bursts of five pulses, with an internal frequency of $500 \mathrm{~Hz}) .^{24}$ Previous studies showed that SCS at higher frequencies $(500 \mathrm{~Hz}$ and $200 \mathrm{~Hz})$ significantly increased cutaneous blood flow in comparison to changes induced by lower frequencies. ${ }^{74,75}$ This suggests that higher frequencies could benefit SCS treatment in PDPN. 
In 2013, Pluijms et al. published the results of a study assessing the effect of SCS frequency (low frequency(LF): $4-10 \mathrm{~Hz} /$ mid frequency(MF): $35-55 \mathrm{~Hz} / \mathrm{high}$ frequency(HF): $150-375 \mathrm{~Hz}$ ) on pain relief in the acute phase of experimental PDPN. ${ }^{65}$ Subsequently, van Beek et al. evaluated the effect of SCS frequency $(5 \mathrm{~Hz} / 50 \mathrm{~Hz} / 500 \mathrm{~Hz})$ on mechanical hypersensitivity in the chronic phase of experimental PDPN. ${ }^{66}$ Although earlier studies suggest that higher frequencies could benefit SCS treatment in PDPN, SCS was equally effective on mechanical hypersensitivity when applied at LF, MF and HF both in the acute phase and in the chronic phase of experimental PDNP. ${ }^{65,66}$ Nevertheless, a delayed wash-in and wash-out effect on mechanical hypersensitivity was noted with use HF-SCS $(500 \mathrm{~Hz})$ in the chronic PDPN animals when compared with LF- $(5 \mathrm{~Hz})$ and MF-SCS $(50 \mathrm{~Hz})$. It was hypothesized that mechanisms by which HF-SCS alleviates pain predominantly result secondary to an effect of SCS on vasodilation, which might explain why alleviation of mechanical hypersensitivity started later as compared with LF- and MFSCS. ${ }^{66}$ Yet, although HF-SCS resulted in higher responder rates as compared to MF-SCS and LF-SCS (70\% versus $65 \%$ and $60 \%$, respectively), HF-SCS did not imply a significant superior pain relieving effect. ${ }^{66}$

There is little data available about the pain relieving effect of different frequency settings in DRGS. Only in one case report and one small pilot study in patients with chronic back pain, a high frequency was applied for DRGS $(2-10 \mathrm{kHz})$ resulting in positive results. ${ }^{76,77}$ In previous DRGS-animal studies conventional frequencies of $20-50 \mathrm{~Hz}$ were used, resulting in a significant reversal of neuropathic pain behavior. ${ }^{64,78} \mathbf{R Q 3}$ provided the background for the study presented in Chapter 5, which aimed to compare effect of the conventionally used MF-DRGS $(20 \mathrm{~Hz})$ - with LF-DRGS $(1 \mathrm{~Hz})-$, HF-DRGS $(1000 \mathrm{~Hz})$ and sham-DRGS on mechanical hypersensivity in the animal model of PDPN. It is reasonable to assume that the increased vasodilatating effect of described in HF-SCS might also occur with the application of HF-DRGS. Therefore, one could hypothesize that HF-DRGS leads to superior pain relief and a higher rate of responders in comparison to DRGS with lower frequencies. However, in the study presented in Chapter 5 HF-DRGS did not lead to a significant superior pain relieving effect in comparison to MF-DRGS and LF-DRGS. ${ }^{79}$ Although HF-DRGS led to higher responder rates compared to MF-DRGS and LF-DRGS (80\% versus $60 \%$ and $50 \%$, respectively), LF-DRGS led to an delayed wash-out effect as compared to MF-DRGS and HF-DRGS. This suggest that LF-DRGS is the most optimal setting in PDPN as compared to MF-DRGS and HF-DRGS. We hypothesize that this difference between SCS and DRGS in experimental PDPN (HF leads to a delayed wash-out effect in SCS in comparison to lower frequencies, ${ }^{66}$ whereas LF leads to a delayed washout effect in DRGS in comparison to higher frequencies ${ }^{79}$ ) might be related to the location of stimulation (DRG versus dorsal column).

It should be taken in consideration that the frequencies used in SCS-studies of PDPN animals ${ }^{65,66}$ differed from those applied in the present DRGS-study and this makes comparison of results and effect of stimulation frequency difficult. It is important to note that the frequency of $1000 \mathrm{~Hz}$ as used in our study for HF-DRGS, in comparison to the 
frequency of $375 \mathrm{~Hz}$ or $500 \mathrm{~Hz}$ as used for HF-SCS, ${ }^{65,66}$ comes closer to the high frequencies as used in clinical settings. ${ }^{80,81}$ In SCS studies HF was limited to $500 \mathrm{~Hz}$ as this frequency was earlier shown to effectively increase SCS induced vasodilatation. ${ }^{74}$ Additionally, at higher frequencies the animals showed signs of discomfort possibly related to use of monophasic pulses with SCS and a monopolar electrode. On the contrary, in the chronic PDPN study of van Beek et al., ${ }^{66}$ and also in the study presented in Chapter 5, biphasic pulses were applied through a quadripolar paddle lead. ${ }^{66}$ The pulse width in all these three studies (Pluijms et al. ${ }^{65}$, van Beek et al. ${ }^{66}$ and the current study) did not differ and was $200 \mu \mathrm{sec}$.

As described above (and in the General Introduction Chapter 1, section 4.1, Spinal Cord Stimulation and section 4.3.2, Analgesic Effect of SCS or DRGS: Mechanism of Action and a Role of GABA), it is known that GABA is a key molecule in the processing and modulation of the nociceptive signal in the spinal dorsal horn with $\mathrm{SCS},{ }^{27,82}$ and GABA has been proposed to have a major role in nociceptive and non-nociceptive processing according to the Gate Control Theory. ${ }^{25}$ From experimental studies it is now concluded that SCS of $A_{\beta}$ fibers in the dorsal column (with conventional settings) results in enhanced GABA release in spinal dorsal horn and this blocks nociceptive fibers. ${ }^{21,22,83-87}$ It was also demonstrated that intrathecal administration of a $G A B A_{B}$ receptor agonist (baclofen) enhances the analgesic effect of SCS. ${ }^{88}$ These experimental findings were translated to the clinic. ${ }^{89-91}$

Detailed understanding of how DRGS in particular may modulate chronic neuropathic pain is still limited. It is likely that DRGS shares some spinal and supraspinal mechanisms with SCS, depending on $A_{\beta}$ fibres activated by both types of stimulation. With DRGS the neurons in the $D R G$, including large $A_{\beta}$ neurons, are stimulated and then this effect might be similar to that observed with $A_{\beta}$ fiber stimulation and antidromic effect in dorsal horn. Therefore, with RQ4 of this thesis the question was addressed whether spinal dorsal horn GABA release is also involved in the mechanism underlying the pain relieving effect of DRGS, likewise SCS. RQ $\mathbf{4}$ is tackled in Chapter 6. We used quantitative immunohistochemical analysis in order to investigate the effect of DRGS on intensity of intracellular GABA-staining levels in the L4-L6 spinal dorsal horn of PDPN animals. In response to RQ4, the results of Chapter 6 indicate that DRGS, on the contrary of SCS, does not result in decreased levels of intracellular GABA-immunoreactivity (GABA-IR) in the spinal dorsal horn. We therefore concluded that the analgesic effect of DRGS is not linked to GABA release in the dorsal horn and the so-called the secondary GABA-mediated Gate. This suggest that the mechanisms underlying (conventional) DRGS in pain relief are different from those of (conventional) SCS. As we now know that key components of the GABA-ergic transmission are expressed in the $D R G,{ }^{92}$ we hypothesize that the pain relieving effect of DRGS in PDPN may act via modulation of the so-called "primary" GABAmediated gate located in the DRG instead of in the "secondary" GABA-mediated gate located in the dorsal horn (see Chapter 1 section 4.3.2). 
In conclusion, this thesis aimed to establish and improve the effectiveness of DRGS in the treatment of pain in painful polyneuropathy (see General Introduction Chapter 1, section 6.1, Aim of the Thesis). Evidence for a pain relieving effect of DRGS in refractory painful polyneuropathy was provided in our clinical studies (Chapter $\mathbf{2}$ and $\mathbf{3}$ ) and then confirmed in a PDPN animal model (Chapter 4). We also concluded that effectiveness for pain relief with DRGS is similar to that observed with SCS (using conventional settings) in PDPN animals. Additionally, we showed that DRGS is equally effective when applied at LF, MF- and HF in an animal model of PDPN. However, LF-DRGS, as compared to MF and HF, resulted in a delayed wash-out effect, suggesting that $L F$ is the most optimal setting in DRGS for PDPN. As lower frequency imply less charge delivered per second, this could have important implications for optimal total charge delivery in clinical practice. With a lower total charge delivery, battery consumption will decreas and at the same time battery life will increase thereby leading to lower health care costs.

Lastly, another key finding of this thesis is that DRGS, in contrast to SCS, does not induce GABA release in spinal dorsal horn of PDPN rats (Chapter 6). With this observation, we hypothesize that the mechanism underlying DRGS-induced pain relief differs from that of dorsal column SCS, and suggest a modulation of a primary GABA mediated Gate control within the DRG to be involved. Further research is warranted to elucidate the mechanism of action underlying DRGS in pain relief.

Based on the results of the studies as presented in this thesis, the following recommendations for future studies and developments can be made:

- Large-scale prospective studies comparing SCS and DRGS in a randomized controlled trial, are needed to prove the efficacy of DRGS in intractable pain associated with polyneuropathy.

- The efficacy of low frequency DRGS should be investigated for effective pain relief in patients with PDPN.

- The hypothesis that DRGS induced pain relief acts via first GABA-ergic Gate in DRG should be tested. 


\section{References}

1. Eldabe S, Espinet A, Wahlstedt A, et al. Retrospective case series on the treatment of painful diabetic peripheral neuropathy with dorsal root ganglion stimulation. Neuromodulation. 2018;21:787-792.

2. Ho KWD, Rempe T, Jerath N, Antony A. Dorsal root ganglion stimulation as a potentially effective treatment for painful hereditary and idiopathic axonal polyneuropathy: a retrospective case series. Neuromodulation. 2020;23:234-238.

3. Slangen R, Schaper NC, Faber CG, et al. Spinal cord stimulation and pain relief in painful diabetic peripheral neuropathy: a prospective two-center randomized controlled trial. Diabetes Care. 2014;37:3016-3024.

4. de Vos CC, Meier K, Zaalberg PB, et al. Spinal cord stimulation in patients with painful diabetic neuropathy: A multicentre randomized clinical trial. Pain. 2014;155:2426-2431.

5. van Beek M, Slangen R, Schaper NC, et al. Sustained treatment effect of spinal cord stimulation in painful diabetic peripheral neuropathy: 24-month follow-up of a prospective two-center randomized controlled trial. Diabetes Care. 2015;38:e132-134.

6. Kumar K, Toth C, Nath RK. Spinal cord stimulation for chronic pain in peripheral neuropathy. Surg Neurol. 1996;46:363-369.

7. Tesfaye S, Watt J, Benbow SJ, Pang KA, Miles J, MacFarlane IA. Electrical spinal-cord stimulation for painful diabetic peripheral neuropathy. Lancet. 1996;348:1698-1701.

8. Daousi C, Benbow SJ, MacFarlane IA. Electrical spinal cord stimulation in the long-term treatment of chronic painful diabetic neuropathy. Diabet Med. 2005;22:393-398.

9. de Vos CC, Rajan V, Steenbergen W, van der Aa HE, Buschman HP. Effect and safety of spinal cord stimulation for treatment of chronic pain caused by diabetic neuropathy. J Diabetes Complicat. 2009;23:40-45.

10. Pluijms WA, Slangen $R$, Bakkers $M$, et al. Pain relief and quality-of-life improvement after spinal cord stimulation in painful diabetic polyneuropathy: A pilot study. Br J Anaesth. 2012;109:623-629.

11. Slangen R, Pluijms WA, Faber CG, Dirksen CD, Kessels AGH, Van Kleef M. Sustained effect of spinal cord stimulation on pain and quality of life in painful diabetic peripheral neuropathy. $\mathrm{Br} J$ Anaesth. 2013;111:1030-1031.

12. Vos C, Meier K, Zaalberg $P$, et al. Spinal cord stimulation in patients with painful diabetic neuropathy: a multicentre randomized clinical trial. Pain. 2014;155:2426-2431.

13. Geurts JW, Joosten EA, Van Kleef M. Current status and future perspectives of spinal cord stimulation in treatment of chronic pain. Pain. 2017;158:771-774.

14. Knezevic NN, Candido KD, Rana S, Knezevic I. The use of spinal cord neuromodulation in the management of HIV-Related Polyneuropathy. Pain Physician. 2015;18:E643-650.

15. Abd-Elsayed A, Schiavoni N, Sachdeva H. Efficacy of spinal cord stimulators in treating peripheral neuropathy: a case series. J Clin Anesth. 2016;28:74-77.

16. Groenen PS, van Helmond N, Chapman KB. Chemotherapy-induced peripheral neuropathy rreated with dorsal root ganglion stimulation. Pain Med. 2018;20:857-859.

17. de La Monte SM, Kril JJ. Human alcohol-related neuropathology. Acta Neuropathol. 2014;127:71-90.

18. Kaku M SD. HIV neuropathy. Curr Opin HIV AIDS. 2014;9:521-526.

19. Colvin LA. Chemotherapy-induced peripheral neuropathy: where are we now? Pain. 2019;160:Suppl 1:S1S10.

20. Yakhnitsa V, Linderoth B, Meyerson BA. Spinal cord stimulation attenuates dorsal horn neuronal hyperexcitability in a rat model of mononeuropathy. Pain. 1999;79:223-233.

21. Stiller CO, Cui JG, O'Connor WT, Brodin E, Meyerson BA, Linderoth B. Release of $Y$-aminobutyric acid in the dorsal horn and suppression of tactile allodynia by spinal cord stimulation in mononeuropathic rats. Neurosurgery. 1996;39:367-375.

22. Cui JG, O'Connor WT, Ungerstedt U, Linderoth B, Meyerson BA. Spinal cord stimulation attenuates augmented dorsal horn release of excitatory amino acids in mononeuropathy via a GABAergic mechanism. Pain. 1997;73:87-95. 


\section{Chapter 7}

23. Ultenius C, Song Z, Lin P, Meyerson BA, Linderoth B. Spinal GABAergic mechanisms in the effects of spinal cord stimulation in a rodent model of neuropathic pain: Is GABA synthesis involved? Neuromodulation. 2013;16:114-120.

24. inderoth B, Foreman RD. Conventional and novel spinal stimulation algorithms: hypothetical mechanisms of action and comments on outcomes. Neuromodulation. 2017;20:525-533.

25. Melzack R, Wall P. Pain mechanisms: a new theory. Science. 1965;150:971-979.

26. Smits $\mathrm{H}$, van Kleef $\mathrm{M}$, Holsheimer J, Joosten EA. Experimental spinal cord stimulation and neuropathic pain: mechanism of action, technical aspects, and effectiveness. Pain Pract. 2013;13:154-168.

27. Guan Y. Spinal cord stimulation: Neurophysiological and neurochemical mechanisms of action. Curr Pain Headache Rep. 2012;16:217-225.

28. West SJ, Bannister K, Dickenson AH, Bennett DL. Circuitry and plasticity of the dorsal horn - toward a better understanding of neuropathic pain. Neuroscience. 2015;300:254-275.

29. Zhang TC, Janik JJ, Grill WM. Mechanisms and models of spinal cord stimulation for the treatment of neuropathic pain. Brain Res. 2014;1569:19-31.

30. Benarroch EE. Dorsal horn circuitry: complexity and implications for mechanisms of neuropathic pain. Neurology. 2016;86:1060-1069.

31. Sivanesan E, Maher DP, Raja SN, Linderoth B, Guan Y. Supraspinal mechanisms of spinal cord stimulation for modulation of pain: five decades of research and prospects for the future. Anesthesiology. 2019;130:651-665

32. Saadé NE, Barchini J, Tchachaghian S, et al. The role of the dorsolateral funiculi in the pain relieving effect of spinal cord stimulation: a study in a rat model of neuropathic pain. Exp Brain Res. 2015;233:10411052.

33. Tazawa T, Kamiya Y, Kobayashi A, et al. Spinal cord stimulation modulates supraspinal centers of the descending antinociceptive system in rats with unilateral spinal nerve injury. Mol Pain. 2015;11:36.

34. Song Z, Ansah OB, Meyerson BA, Pertovaara A, Linderoth B. Exploration of supraspinal mechanisms in effects of spinal cord stimulation: role of the locus coeruleus. Neuroscience. 2013;253:426-434.

35. Barchini J, Tchachaghian S, Shamaa F, et al. Spinal segmental and supraspinal mechanisms underlying the pain-relieving effects of spinal cord stimulation: an experimental study in a rat model of neuropathy. Neuroscience. 2012;215:196-208.

36. Deogaonkar M, Sharma M, Oluigbo C, et al. Spinal cord stimulation (SCS) and runctional magnetic resonance imaging ( $\mathrm{FMRI}$ ): modulation of cortical connectivity with therapeutic SCS. Neuromodulation. 2016;19:142-153.

37. Moens $M$, Mariën $P$, Brouns R, et al. Spinal cord stimulation modulates cerebral neurobiology: a proton magnetic resonance spectroscopy study. Neuroradiology. 2013;55:1039-1047.

38. Bentley LD, Duarte R V., Furlong PL, Ashford RL, Raphael JH. Brain activity modifications following spinal cord stimulation for chronic neuropathic pain: a systematic review. Eur J Pain. 2016;20:499-511.

39. Ubbink DT, Vermeulen H. Spinal cord stimulation for non-reconstructable chronic critical leg ischaemia. Cochrane Database Syst Rev. 2013;(2):CD004001.

40. Ubbink DT, Vermeulen H. Spinal cord stimulation for critical leg ischemia: a review of effectiveness and optimal patient selection. J Pain Symptom Manage. 2006;31(4 Suppl):S30-35.

41. Deer TR, Mekhail N, Provenzano D, et al. The appropriate use of neurostimulation of the spinal cord and peripheral nervous system for the treatment of chronic pain and ischemic diseases: The neuromodulation appropriateness consensus committee. Neuromodulation. 2014;17:515-550.

42. van Beek M, Hermes D, Honig WM, et al. Long-term spinal cord stimulation alleviates mechanical hypersensitivity and increases peripheral cutaneous blood perfusion in experimental painful diabetic polyneuropathy. Neuromodulation. 2018;21:472-479.

43. van Beek M. Thesis: Spinal Cord Stimulation in Clinical and experimental painful diabetic polyneuropathy. Maastricht University. 2017.

44. Tanaka S, Barron KW, Chandler MJ, Linderoth B, Foreman RD. Low intensity spinal cord stimulation may induce cutaneous vasodilation via CGRP release. Brain Res. 2001;896:183-187. 
45. Wu M, Komori N, Qin C, Farber JP, Linderoth B, Foreman RD. Roles of peripheral terminals of transient receptor potential vanilloid-1 containing sensory fibers in spinal cord stimulation-induced peripheral vasodilation. Brain Res. 2007;1156:80-92.

46. Wu M, Linderoth B, Foreman RD. Putative mechanisms behind effects of spinal cord stimulation on vascular diseases: A review of experimental studies. Auton Neurosci Basic Clin. 2008;138:9-23.

47. Linderoth B, Herregodts $P$, Meyerson BA. Sympathetic mediation of peripheral vasodilation induced by spinal cord stimulation: animal studies of the role of cholinergic and adrenergic receptor subtypes. Neurosurgery. 1994;35:711-719.

48. Tanaka S, Komori N, Barron KW, Chandler MJ, Linderoth B, Foreman RD. Mechanisms of sustained cutaneous vasodilation induced by spinal cord stimulation. Auton Neurosci Basic Clin. 2004;114:55-60.

49. Chang Chien GC, Mekhail N. Alternate intraspinal targets for spinal cord stimulation: a systematic review. Neuromodulation. 2017;20:629-641.

50. Sapunar D, Kostic S, Banozic A, Puljak L. Dorsal root ganglion - a potential new therapeutic target for neuropathic pain. J Pain Res. 2012;5:31-38.

51. McCallum JB, Kwok W-M, Sapunar D, Fuchs A, Hogan QH. Painful peripheral nerve injury decreases calcium current in axotomized sensory neurons. Anesthesiology. 2006;105:160-168.

52. Rush AM, Dib-Hajj SD, Liu S, Cummins TR, Black JA, Waxman SG. A single sodium channel mutation produces hyper- or hypoexcitability in different types of neurons. Proc Natl Acad Sci. 2006;103:8245-8250.

53. Devor M. Neuropathic pain: What do we do with all these theories? Acta Anaesthesiol Scand. 2001;45:1121-1127.

54. Koopmeiners AS, Mueller S, Kramer J, Hogan QH. Effect of electrical field stimulation on dorsal root ganglion neuronal function. Neuromodulation. 2013;16:304-311; discussion 310-11.

55. Lee BH, Yoon YW, Chung K, Chung JM. Comparison of sympathetic sprouting in sensory ganglia in three animal models of neuropathic pain. Exp Brain Res. 1998;120:432-438.

56. Xie W, Strong JA, Zhang JM. Increased excitability and spontaneous activity of rat sensory neurons following in vitro stimulation of sympathetic fiber sprouts in the isolated dorsal root ganglion. Pain. 2010;151:447-459.

57. Ramer MS, Bisby MA. Rapid sprouting of sympathetic axons in dorsal root ganglia of rats with a chronic constriction injury. Pain. 1997;70:237-244.

58. Miller JP, Eldabe S, Buchser E, Johanek LM, Guan Y, Linderoth B. Parameters of spinal cord stimulation and their role in electrical charge delivery: a review. Neuromodulation. 2016;19:373-384.

59. Liem L, Russo M, Huygen FJPM, et al. A multicenter, prospective trial to assess the safety and performance of the spinal modulation dorsal root ganglion neurostimulator system in the treatment of chronic pain. Neuromodulation. 2013:471-482.

60. Liem L, Russo M, Huygen FJPM, et al. One-year outcomes of spinal cord stimulation of the dorsal root ganglion in the treatment of chronic neuropathic pain. Neuromodulation. 2015;18:41-49.

61. Schu S, Gulve A, Eldabe S, et al. Spinal cord stimulation of the dorsal root ganglion for groin pain-a retrospective review. Pain Pract. 2015;15:293-299.

62. Deer TR, Levy RM, Kramer J, et al. Dorsal root ganglion stimulation yielded higher treatment success rate for complex regional pain syndrome and causalgia at 3 and 12 months: A randomized comparative trial. Pain. 2017;158:669-681.

63. Galer BS, Gianas A, Jensen MP. Painful diabetic polyneuropathy: epidemiology, pain description, and quality of life. Diabetes Res Clin Pract. 2000;47:123-128.

64. Pan B, Yu H, Fischer GJ, Kramer JM, Hogan QH. Dorsal root ganglionic field stimulation relieves spontaneous and induced neuropathic pain in rats. J Pain. 2016;17:1349-1358.

65. Pluijms WA, van Kleef $M$, Honig WM, Janssen SP, Joosten EA. The effect of spinal cord stimulation frequency in experimental painful diabetic polyneuropathy. Eur J Pain. 2013;17:1338-1346.

66. Beek $M$, Kleef $M$, Linderoth $B$, et al. Spinal cord stimulation in experimental chronic painful diabetic polyneuropathy: delayed effect of High-frequency stimulation. Eur J Pain. 2017;21:795-803. 


\section{Chapter 7}

67. Tesfaye S, Selvarajah D. Advances in the epidemiology, pathogenesis and management of diabetic peripheral neuropathy. Diabetes Metab Res Rev. 2012;28(SUPPL. 1):8-14.

68. Graham RD, Bruns TM, Duan B, Lempka SF. Dorsal root ganglion stimulation for chronic pain modulates A $\beta$-fiber activity but not C-fiber activity: A computational modeling study. Clin Neurophysiol. 2019;130:941-951.

69. Kramer J, Liem L, Russo M, Smet I, Van Buyten JP, Huygen F. Lack of body positional effects on paresthesias when stimulating the dorsal root ganglion (DRG) in the treatment of chronic pain. Neuromodulation. 2015;18:50-57.

70. Holsheimer J. Which neuronal elements are activated directly by spinal cord stimulation. Neuromodulation. 2002;5:25-31.

71. Brierley JB. The penetration of particulate matter from the cerebrospinal fluid into the spinal ganglia, peripheral nerves, and perivascular spaces of the central nervous system. J Neurol Neurosurg Psychiatry. 1950;13:203-215.

72. Deer TR, Grigsby E, Weiner RL, Wilcosky B, Kramer JM. A prospective study of dorsal root ganglion stimulation for the relief of chronic pain. Neuromodulation. 2013;16:67-72.

73. Meuwissen KPV, Gu JW, Zhang TC, Joosten EA. Conventional-SCS vs. burst-SCS and the behavioral effect on mechanical hypersensitivity in a rat model of chronic neuropathic pain: effect of amplitude. Neuromodulation. 2018;21:19-30.

74. Gao J, Wu M, Li L, et al. Effects of spinal cord stimulation with "standard clinical" and higher frequencies on peripheral blood flow in rats. Brain Res. 2010;1313:53-61.

75. Kao CH, Chen JJ, Hsu YM, Bau DT, Yao CH, Chen YS. High-frequency electrical stimulation can be a complementary therapy to promote nerve regeneration in diabetic rats. PLoS One. 2013;8:e79078.

76. Billet B, Wynendaele R, Vanquathem NE. Wireless neuromodulation for chronic back pain: delivery of highfrequency dorsal root ganglion stimulation by a minimally invasive technique. Case Rep Med. 2017;2017:4203271.

77. Billet B, Hanssens K, De Coster O, et al. Wireless high-frequency dorsal root ganglion stimulation for chronic low back pain: A pilot study. Acta Anaesthesiol Scand. 2018 Apr 19. [Epub ahead of print].

78. Koetsier E, Franken G, Debets J, et al. Effectiveness of dorsal root ganglion stimulation and dorsal column spinal cord stimulation in a model of experimental painful diabetic polyneuropathy. CNS Neuroscience and Therapeutics. 2018;25:367-374.

79. Koetsier E, Franken G, Debets J, et al. Dorsal root ganglion stimulation in experimental painful diabetic polyneuropathy: delayed wash-out of pain relief after low-frequency $(1 \mathrm{~Hz})$ stimulation. Neuromodulation. 2019;23:177-184.

80. Thomson SJ, Tavakkolizadeh M, Love-Jones S, et al. Effects of rate on analgesia in kilohertz frequency spinal cord stimulation: results of the PROCO randomized controlled trial. Neuromodulation. 2018;21:67-76.

81. Kapural L, Yu C, Doust MW, et al. Novel 10-kHz high-frequency therapy (HF10 Therapy) is superior to traditional low-frequency spinal cord stimulation for the treatment of chronic back and leg pain. Anesthesiology. 2015;123:851-860

82. Guan Y, Wacnik PW, Yang F, et al. Spinal cord stimulation-induced analgesia: Electrical stimulation of dorsal column and dorsal roots attenuates dorsal horn neuronal excitability in neuropathic rats. Anesthesiology. 2010;113:1392-1405.

83. Linderoth B, Stiller CO, Gunasekera L, O'Connor WT, Ungerstedt U, Brodin E. Gamma-aminobutyric acid is released in the dorsal horn by electrical spinal cord stimulation: an in vivo microdialysis study in the rat. Neurosurgery. 1994;34:484-488; discussion 488-489.

84. Janssen SP, Gerard S, Raijmakers ME, Truin M, Van Kleef M, Joosten EA. Decreased intracellular GABA levels contribute to spinal cord stimulation-induced analgesia in rats suffering from painful peripheral neuropathy: the role of KCC2 and GABAAreceptor-mediated inhibition. Neurochem Int. 2012;60:21-30.

85. Eaton MJ, Plunkett JA, Karmally S, Martinez MA, Montanez K. Changes in GAD- and GABAimmunoreactivity in the spinal dorsal horn after peripheral nerve injury and promotion of recovery by lumbar transplant of immortalized serotonergic precursors. J Chem Neuroanat. 1998;16:57-72. 
86. Kim J, Kim SJ, Lee H, Chang JW. Effective neuropathic pain relief through sciatic nerve administration of GAD65-expressing rAAV2. Biochem Biophys Res Commun. 2009;388:73-78.

87. Moore KA, Kohno T, Karchewski LA, Scholz J, Baba H, Woolf CJ. Partial peripheral nerve injury promotes a selective loss of GABAergic inhibition in the superficial dorsal horn of the spinal cord. J Neurosci. 2002;22:6724-6731.

88. Cui JG, Linderoth B, Meyerson BA. Effects of spinal cord stimulation on touch-evoked allodynia involve GABAergic mechanisms. An experimental study in the mononeuropathic rat. Pain. 1996;66:287-295.

89. Lind G, Meyerson BA, Winter J, Linderoth B. Intrathecal baclofen as adjuvant therapy to enhance the effect of spinal cord stimulation in neuropathic pain: a pilot study. Eur J Pain. 2004;8:377-383.

90. Lind G, Schechtmann G, Winter J, Meyerson BA, Linderoth B. Baclofen-enhanced spinal cord stimulation and intrathecal baclofen alone for neuropathic pain: long-term outcome of a pilot study. Eur J PAIN. 2008;12:132-136.

91. Schechtmann G, Lind G, Winter J, Meyerson BA, Linderoth B. Intrathecal clonidine and baclofen enhance the pain-relieving effect of spinal cord stimulation: a comparative placebo-controlled, randomized trial. Neurosurgery. 2010;67:173-181.

92. Du X, Hao H, Yang Y, et al. Local GABAergic signaling within sensory ganglia controls peripheral nociceptive transmission. J Clin Invest. 2017;127:1741-1756. 

chapter 8

Valorization Addendum 


\section{Valorization addendum}

In this addendum, we will attempt to translate the findings of this thesis in terms of 'knowledge valorization'. Knowledge valorization of research refers to the process of creating value from knowledge, by making it available for social and or societal utilization. Diseases associated with chronic pain are increasing in prevalence and are a global cause of disability in both the developed and developing countries. ${ }^{1,2}$ Chronic pain is associated with a lowered quality of life $(\mathrm{QoL}))^{3,4}$ and can have an important impact upon mood, cognition and emotional functioning, ${ }^{5}$ leading to restrictions upon functioning during daily activities and work. ${ }^{6}$ Amongst all causes of chronic pain, neuropathic pain is particularly cumbersome. ${ }^{7}$ Additionally, neuropathic pain is widely recognized as one of the most difficult pain syndromes to manage. ${ }^{8}$

One of the most common causes of neuropathic pain is polyneuropathy, which is typically characterized by symmetrical sensory symptoms in the distal parts of the limbs. ${ }^{9}$ The overall prevalence of polyneuropathy in the general population ranges from 1 to 3\% and rises to $7 \%$ in the elderly. ${ }^{10}$ In developing countries the prevalence is lower, possibly explained by a smaller proportion of elderly and by differences in the prevalence of polyneuropathy risk factors. ${ }^{10}$ In the last decades, the prevalence of polyneuropathy is increasing due to an aging population and the increasing prevalence of risk factors like diabetes mellitus (DM) and obesity. ${ }^{10,11}$ The prevalence of neuropathic pain in diabetic polyneuropathy in people with DM ranges from 6 to 34\%, ${ }^{12}$ and this symptom is its most costly complication. ${ }^{13-15}$ Other causes of polyneuropathy include toxic agents, such as chemotherapeutic drugs, nutritional deficiencies, autoimmune-mediated causes and hereditary factors. ${ }^{9,10}$ Polyneuropathy is furthermore reported to be present in 13-66 \% of chronic alcoholics, ${ }^{16,17}$ and in up to $50 \%$ an underlying cause cannot be identified. ${ }^{18}$

In this chapter, we will address the social and economic relevance of our research results. For this purpose, will we first present the impact of pain on patients with polyneuropathy and its impact on society.

\section{What is the impact of pain on patients with polyneuropathy?}

Painful polyneuropathy has a huge impact on patients. Despite other symptoms like paresthesias and dysesthesias, pain is a primary indicator for worsening QoL and diminished overall wellbeing in patients with polyneuropathy..$^{19}$ A study regarding PDPN patients showed that PDPN patients had a significantly worse QoL compared with DM patients without pain and DM patients with non-neuropathic pain. ${ }^{20} \mathrm{QoL}$ is inversely associated with neuropathic pain severity and pain duration in patients with painful polyneuropathy. ${ }^{21}$ The etiology of polyneuropathy does not influence levels of neuropathic pain-related compromise of QoL. ${ }^{19}$ 
Painful polyneuropathy is a disabling disease and is related to poor sleep and symptoms of anxiety and depression. ${ }^{19,20,22}$ Pain intensity in polyneuropathy patients is positively associated with disability. ${ }^{22}$ Pain in these patients can considerably affect daily life by reducing the ability to walk and perform general everyday activities. ${ }^{12}$ It can have a major impact on recreational activities, work, social activities, mobility, and experienced stress. $^{23,24}$ Studies have demonstrated that PDPN patients score 4.8 for overall pain interference on the brief pain inventory (BPI) $(0$ is no interference and 10 is complete interference). ${ }^{12}$ The subscales for general activity and walking ability were generally most affected. ${ }^{12,25}$ However, the subscales for sleep, mood and enjoyment of life were almost equally highly impaired. ${ }^{25}$ Painful polyneuropathy is often associated with sleep disturbance due to the fact that most patients have nocturnal pain. Studies of PDPN patients report that $72-96 \%$ of the patients are moderately to severely affected in their sleep., ${ }^{26}$ Higher pain severity is significantly correlated with higher interference of sleep. ${ }^{12,27}$ In addition, studies have shown that $24.5-72.1 \%$ of PDPN patients have symptoms of depression and/or anxiety pain, ${ }^{12}$ and PDPN has been shown to be a greater determinant of depression than other DM-related complications and comorbidities. ${ }^{28}$

Despite this great patient burden of pain caused by polyneuropathy, pain in polyneuropathy patients is often not well recognized and treated. ${ }^{29}$ The effectiveness of pharmacological treatment is often minor and frequently accompanied by unacceptable side effects. ${ }^{30}$ In addition, patients with painful polyneuropathy are more likely to have medication incompliance, like inappropriate polypharmacy and misuse of prescribed medications. ${ }^{19}$ Many patients require pain treatment with more than one medication, which increases the risk of additional adverse events and incorrect use of medication. ${ }^{31}$ Furthermore, concomitant medication use is high in these patients. For instance, a patient survey has shown that $43 \%$ of PDPN patients received prescription medications for sleep disturbance, anxiety and/or depression. ${ }^{32}$

\section{What is the impact of pain in patients with polyneuropathy on society?}

Painful polyneuropathy is associated with greater health care utilization in comparison to not painful polyneuropathy. Examples of health care utilization are outpatient clinic visitations, overnight hospitalizations, and the use of a nursing or rehabilitation home. ${ }^{19}$ To give an example, the resource use of PDPN patients results in mean annual per patient UK health care costs of $€ 2,963 .{ }^{33}$ Of these costs, $41 \%$ is accounted for inpatient care. The annual costs found in the UK were comparable to those found in Spain. ${ }^{34}$ The costs of medications account for approximately $30 \%$ of the healthcare costs. ${ }^{33,34}$ In addition, in patients with painful polyneuropathy a large number 
of hospital admissions are related to medication side effects. ${ }^{19}$ Higher pain severity in PDPN patients is significantly correlated with higher resource use and direct costs. ${ }^{25,33,35}$

Painful polyneuropathy can result in additional costs for society because it can lead to absence from work or reduced functionality at work..$^{12}$ In a European study regarding 634 PDPN patients, disturbance in employment status resulted in productivity losses of $€ 10,484$ per patient per year. ${ }^{36}$ PDPN severity was significantly associated with lost productivity, which was higher with increasing severity, and resulted in significantly higher costs. Productivity losses were similar among the countries France, Germany, Italy, and the UK, and were primarily driven by presenteism (impairment while working). In a UK study, 35\% of the PDPN patients reported disruption in employment status due to pain, and $59 \%$ of the working patients was less productive at work. ${ }^{25}$

Generally, neuropathic pain reduction, as a result of treatment, is related to improvement in QoL. ${ }^{37}$

The humanistic and economic burden from painful polyneuropathy appears to be higher with increasing pain severity. Therefore, it is of particular importance to recognize painful polyneuropathy in patients and to optimize pain treatment. ${ }^{19}$ Emerging neuromodulation options for painful polyneuropathy are spinal cord stimulation (SCS) and dorsal root ganglion stimulation (DRGS), ${ }^{38-42}$ being a last resort treatment method when conventional therapies have failed. ${ }^{38,39,43,44}$ SCS has been shown to be effective in PDPN. ${ }^{38,45-49}$ Treatment with SCS has also been shown to be effective in intractable painful polyneuropathy due to other causes, like HIV infection and chemotherapy. ${ }^{43,44}$ Slangen et al. performed an economic evaluation comparing SCS with best medical treatment (BMT) in PDPN patients. ${ }^{50}$ Incremental cost-effectiveness ratios were based on: 1) societal costs and quality-adjusted life years, and 2) direct health care costs and the number of successfully treated patients, respectively. Although SCS was considerably more effective compared with BMT, Slangen et al. concluded from the results of their study that SCS was not cost effective compared with BMT at the 12-month follow-up, mainly because of the substantial initial investment costs of SCS. However, secondary analyses showed that the incremental cost effectiveness ratios decreased considerably when correcting for baseline differences in costs, and extending the depreciation period of the SCS material to 4 years. ${ }^{50}$

There are limits to the effectiveness of conventional SCS in the treatment of painful polyneuropathy. To illustrate, this treatment is known to provide approximately $50 \%$ pain reduction to only $60 \%$ of PDPN patients. ${ }^{38,45,46,49}$ Forty percent of the patients is therefore not responding to this therapy. Furthermore, the analgesic effect of SCS treatment is known to decrease over time, ${ }^{51}$ and SCS is often unable to cover the pain in the so-called "difficult-to-reach areas", like the feet. ${ }^{52,53}$ In view of these limitations, optimization of neurostimulation therapy is needed and the new location of stimulation at the DRG (dorsal root ganglion stimulation, DRGS) is shown to be a promising new option for treatment of PDPN. Early findings from one retrospective case series in painful diabetic polyneuropathy patients suggested that DRGS is an effective neuromodulation modality 
to improve painful symptoms. ${ }^{41}$ Furthermore, the results of another small retrospective case series suggest that DRGS may be an effective treatment option for painful hereditary and idiopathic axonal polyneuropathy. ${ }^{42}$ Except the fact that DRGS seems provide a better coverage of the difficult-to-reach areas, it offers several other potential benefits over SCS systems like lack of positional and movement effects on stimulation and reduced migration rate, because of better lead stability. ${ }^{52,54}$ Additionally, as the anatomical location of the DRG offers a closer proximity to the electrodes compared to the spinal cord and its dorsal columns, reduced power is required. ${ }^{52,54}$ Nevertheless, more clinical evidence is warranted to confirm the efficacy of this treatment for painful polyneuropathy.

\section{What is the social and economic relevance of our research results?}

If DRGS can effectively reduce pain in intractable painful polyneuropathy, not only the individual patient would benefit, but also the burden on medical care systems would be lessened. Hence, the research described in this thesis focused upon establishment and optimization of treatment with DRGS in painful polyneuropathy patients, and further understanding the underlying mechanism.

A major conclusion of the results in this thesis is that DRGS seems to be established for pain relief in painful polyneuropathy in humans and in a PDPN animal model (Chapter 2, 3 and 4). Our results furthermore showed that effectiveness for pain relief is similar with DRGS and SCS in PDPN animals (Chapter 4).

To further optimize effectiveness of DRGS in the treatment of pain in painful polyneuropathy, we explored and compared the effectiveness of different DRGS frequencies and concluded that DRGS is equally effective when applied at low-, mid- and high-frequency, at least in an animal model of PDPN (Chapter 5). Nevertheless, as lowfrequency DRGS resulted in a delayed wash-out effect, this frequency might be the most optimal setting in DRGS for PDPN as compared to mid frequency and high frequency. Moreover, the use of LF-DRGS will lead to a prolonged battery life as compared to MFand HF-DRGS and thus has important consequences for costs-effectiveness of this treatment.

Lastly, understanding of the working mechanism underlying SCS- and DRGS-induced pain relief may enable optimization of treatment and result in better treatment outcomes. In relation to this, another key finding of this thesis is that DRGS, in contrast to SCS, does not induce $\mathrm{y}$-aminobutyric acid (GABA) release in spinal dorsal horn of PDPN rats (Chapter 6 ). With this observation we suggest that the mechanism underlying DRGSinduced pain relief is different from that of dorsal column SCS and the modulation of a GABA mediated "Gate Control" in the DRG, functioning as a prime Gate of nociception, is 
suggested. Further research is warranted to elucidate the mechanism underlying DRGS in pain relief.

To conclude, painful polyneuropathy patients are subjected to a significant physiological, psychological and functional burden. It is essential to raise awareness of painful polyneuropathy and to encourage healthcare providers to better identify patients with painful polyneuropathy to, as this condition deeply affects patients's quality of life and disability. If new treatment options like DRGS can effectively reduce pain in painful polyneuropathy patient, the humanistic and economic burden of painful polyneuropathy would be lessened. With the research of this thesis, we established and tried to optimize DRGS in the treatment of painful polyneuropathy patients. Furthermore, our research let us a little bit closer to understanding the underlying mechanism of DRGS, hopefully enabling future optimization of treatment, resulting in better outcomes. 


\section{References}

1. Vos T, Barber RM, Bell B, et al. Global, regional, and national incidence, prevalence, and years lived with disability for 301 acute and chronic diseases and injuries in 188 countries, 1990-2013: A systematic analysis for the Global Burden of Disease Study 2013. Lancet. 2015;388:1603-1658.

2. Rice AS, Smith BH, Blyth FM. Pain and the global burden of disease. Pain. 2016;157:791-796.

3. Smith BH, Torrance N, Bennett MI, Lee AJ. Health and quality of life associated with chronic pain of predominantly neuropathic origin in the community. Clin J Pain. 2007;23:143-149.

4. Gormsen L, Rosenberg R, Bach FW, Jensen TS. Depression, anxiety, health-related quality of life and pain in patients with chronic fibromyalgia and neuropathic pain. Eur J Pain. 2010;14:127.e1-8.

5. Turk DC. The role of psychological factors in chronic pain. In: Acta Anaesthesiologica Scandinavica. 1999 ;17(9 Suppl):T70-92.

6. Becker N, Thomsen AB, Olsen AK, Sjøgren P, Bech P, Eriksen J. Pain epidemiology and health related quality of life in chronic non-malignant pain patients referred to a Danish multidisciplinary pain center. Pain. 1997;73:393-400.

7. Toth $\mathrm{C}, \mathrm{Au}$ S. A prospective identification of neuropathic pain in specific chronic polyneuropathy syndromes and response to pharmacological therapy. Pain. 2008;138:657-666.

8. Van Hecke O, Austin SK, Khan RA, Smith BH, Torrance N. Neuropathic pain in the general population: A systematic review of epidemiological studies. Pain. 2014;155:654-662.

9. Callaghan BC, Price RS, Feldman EL. Distal symmetric polyneuropathy a review. JAMA. 2015;314:21722181.

10. Hanewinckel R, van Oijen M, Ikram MA, van Doorn PA. The epidemiology and risk factors of chronic polyneuropathy. Eur J Epidemiol. 2016;31:5-20.

11. Gregg EW, Sorlie P, Paulose-Ram R, et al. Prevalence of lower-extremity disease in the U.S. adult population $\geq 40$ years of age with and without diabetes: 1999-2000 National Health and Nutrition Examination Survey. Diabetes Care. 2004;27:1591-1597.

12. Alleman $\mathrm{CJ}$, Westerhout $\mathrm{KY}$, Hensen $\mathrm{M}$, et al. Humanistic and economic burden of painful diabetic peripheral neuropathy in Europe: A review of the literature. Diabetes Res Clin Pract. 2015;109:215-225.

13. England JD, Asbury AK. Peripheral neuropathy. Lancet. 2004;363:2151-2161.

14. Burns TM, Mauermann ML. The evaluation of polyneuropathies. Neurology. 2011;76 (7 Suppl 2):S6-13.

15. Peltier A, Goutman SA, Callaghan BC. Painful diabetic neuropathy. BMJ. 2014;348:g1799.

16. Koike H, Sobue G. Alcoholic neuropathy. Curr Opin Neurol. 2006;19:481-486.

17. Mellion M, Gilchrist JM, De La Monte S. Alcohol-related peripheral neuropathy: Nutritional, toxic, or both? Muscle and Nerve. 2011;43:309-316.

18. Bennett DL, Woods CG. Painful and painless channelopathies. Lancet Neurol. 2014;13:587-599.

19. Poliakov I, Toth C. The impact of pain in patients with polyneuropathy. Eur J Pain. 2011;15:1015-1022.

20. Davies M, Brophy S, Williams R, Taylor A. The prevalence, severity, and impact of painful diabetic peripheral neuropathy in type 2 diabetes. Diabetes Care. 2006;29:1518-1522.

21. Currie CJ, Poole CD, Woehl A, et al. The health-related utility and health-related quality of life of hospitaltreated subjects with type 1 or type 2 diabetes with particular reference to differing severity of peripheral neuropathy. Diabetologia. 2006;49:2272-2280.

22. Geelen CC, Smeets RJEM, Schmitz S, van den Bergh JP, Goossens MEJB, Verbunt JA. Anxiety affects disability and quality of life in patients with painful diabetic neuropathy. Eur J Pain (United Kingdom). 2017;21:1632-1641.

23. Jensen MP, Chodroff MJ, Dworkin RH. The impact of neuropathic pain on health-related quality of life: Review and implications. Neurology. 2007;68:1178-1182.

24. Galer BS, Gianas A, Jensen MP. Painful diabetic polyneuropathy: Epidemiology, pain description, and quality of life. Diabetes Res Clin Pract. 2000;47:123-128.

25. Tölle T, Xu X, Sadosky AB. Painful diabetic neuropathy: A cross-sectional survey of health state impairment and treatment patterns. J Diabetes Complications. 2006;20:26-33. 


\section{Valorization Addendum}

26. Selvarajah D, Thomas L, Cash T, et al. A biopsychosocial examination of factors contributing to sleep impairment in painful diabetic neuropathy. Diabet Med. 2011;11:218-225.

27. Cash TC, Selvarajah D, Roddick A, Thomas L, Gandhi R, Tesfaye S. A population-based study of the relationship between neuropathic pain severity and important patient-related health outcomes in diabetic neuropathy: Time to revaluate current clinical practice? Diabet Med. 2012;29(Suppl. 1).

28. D'Amato C, Morganti R, Di Gennaro F, et al. Neuropathic pain impact on sleep and circadian rhythm of blood pressure in painful diabetic polyneuropathy. J Peripher Nerv Syst: JPNS 2012;17:519 (Suppl).

29. Gore M, Brandenburg NA, Hoffman DL, Tai KS, Stacey B. Burden of Illness in Painful Diabetic Peripheral Neuropathy: The Patients' Perspectives. J Pain. 2006; 7:892-900.

30. Bril V, England J, Franklin GM, et al. Evidence-based Guideline: treatment of Painful Diabetic Neuropathy. PM\&R. 2011;3:345-352, 352.e1-21.

31. Kerr L. Polypharmacy in diabetes and solutions for greater adherence. Pract Diabetes. 2009;26:289-291.

32. Rodríguez MJ, Díaz S, Vera-Llonch M, Dukes E, Rejas E A J. Cost-effectiveness analysis of pregabalin versus gabapentin in the management of neuropathic pain due to diabetic polyneuropathy or post-herpetic neuralgia. Curr Med Res Opin. 2007;23:2585-2596.

33. Currie CJ, Poole CD, Woehl A, et al. The financial costs of healthcare treatment for people with Type 1 or Type 2 diabetes in the UK with particular reference to differing severity of peripheral neuropathy. Diabet Med. 2007;24:187-194.

34. De Salas-Cansado M, Pérez C, Saldaña MT, et al. An economic evaluation of pregabalin versus usual care in the management of community-treated patients with refractory painful diabetic peripheral neuropathy in primary care settings. Prim Care Diabetes. 2012;6:303-312.

35. Sadosky A, Schaefer C, Mann R, et al. Burden of illness associated with painful diabetic peripheral neuropathy among adults seeking treatment in the US: Results from a retrospective chart review and cross-sectional survey. Diabetes, Metab Syndr Obes Targets Ther. 2013;6:79-92.

36. Taylor-Stokes G, Pike J, Sadosky A, Chandran A, Toelle T. Impact of patient-rated severity of painful diabetic peripheral neuropathy on productivity in the european setting. Pain Pract. 2012;12 (Supple. 1): S111-112.

37. Deshpande MA, Holden RR, Gilron I. The impact of therapy on quality of life and mood in neuropathic pain: What is the effect of pain reduction? Anesth Analg. 2006;102:1473-1479.

38. Slangen R, Schaper NC, Faber CG, et al. Spinal cord stimulation and pain relief in painful diabetic peripheral neuropathy: A prospective two-center randomized controlled trial. Diabetes Care. 2014;37:3016-3024.

39. de Vos CC, Meier K, Zaalberg PB, et al. Spinal cord stimulation in patients with painful diabetic neuropathy: A multicentre randomized clinical trial. Pain. 2014;155:2426-2431.

40. van Beek M, Geurts JW, Slangen R, et al. Severity of neuropathy is associated with long-term spinal cord stimulation outcome in painful diabetic peripheral neuropathy: Five-year follow-up of a prospective twocenter clinical trial. Diabetes Care. 2018;41:32-38.

41. Eldabe S, Espinet A, Wahlstedt A, et al. Retrospective case series on the treatment of painful diabetic peripheral neuropathy with dorsal root ganglion stimulation. Neuromodulation. 2018;21:787-792.

42. Ho KWD, Rempe T, Jerath N, Antony A. Dorsal root ganglion stimulation as a potentially effective treatment for painful hereditary and idiopathic axonal polyneuropathy: a retrospective case series. Neuromodulation. 2020;23:234-238.

43. Abd-Elsayed A, Schiavoni N, Sachdeva H. Efficacy of spinal cord stimulators in treating peripheral neuropathy: a case series. J Clin Anesth. 2016;28:74-77.

44. Knezevic NN, Candido KD, Rana S, Knezevic I. The use of spinal cord neuromodulation in the management of HIV-related polyneuropathy. Clin J Sport Med. 2016;18:E643-50.

45. de Vos CC, Rajan V, Steenbergen W, van der Aa HE, Buschman HP. Effect and safety of spinal cord stimulation for treatment of chronic pain caused by diabetic neuropathy. J Diabetes Complicat. 2009;23:40-45.

46. Pluijms WA, Slangen $\mathrm{R}$, Bakkers $\mathrm{M}$, et al. Pain relief and quality-of-life improvement after spinal cord stimulation in painful diabetic polyneuropathy: A pilot study. Br J Anaesth. 2012;109:623-629. 
47. van Beek M, Geurts JW, Slangen R, et al. Severity of neuropathy is associated with long-term spinal cord stimulation outcome in painful diabetic peripheral neuropathy: Five-year follow-up of a prospective twocenter clinical trial. Diabetes Care. 2018;41:32-38.

48. Slangen R, Pluijms WA, Faber CG, Dirksen CD, Kessels AGH, Van Kleef M. Sustained effect of spinal cord stimulation on pain and quality of life in painful diabetic peripheral neuropathy. $\mathrm{Br} J$ Anaesth. 2013;111:1030-1031.

49. Vos C, Meier K, Zaalberg P, et al. Spinal cord stimulation in patients with painful diabetic neuropathy: a multicentre randomized clinical trial. Pain. 2014;155:2426-2431.

50. Slangen R, Faber CG, Schaper NC, et al. A trial-based economic evaluation comparing spinal cord stimulation with best medical treatment in painful diabetic peripheral neuropathy. J Pain. 2017;18:405414.

51. van Beek M, Geurts JW, Slangen R, et al. Severity of neuropathy is associated with long-term spinal cord stimulation outcome in painful diabetic peripheral neuropathy: Five-year follow-up of a prospective twocenter clinical trial. Diabetes Care. 2018;41:32-38.

52. Liem L, Russo M, Huygen FJ, et al. A multicenter, prospective trial to assess the safety and performance of the spinal modulation dorsal root ganglion neurostimulator system in the treatment of chronic pain. Neuromodulation. 2013:471-482.

53. Verrills P, Sinclair C, Barnard A. A review of spinal cord stimulation systems for chronic pain. J Pain Res. 2016;9:481-492.

54. Tesfaye S, Selvarajah D. Advances in the epidemiology, pathogenesis and management of diabetic peripheral neuropathy. Diabetes Metab Res Rev. 2012;28(SUPPL. 1):8-14. 



\section{Co-author Affiliations}

E.A. Joosten

J.A.M. van Zundert

S.M.J. van Kuijk

R.S.G.M. Perez

P. Maino

J. Dukanac

G. Franken

B. Linderoth

J. Debets

L. Heijmans
Department of Anesthesiology and Pain Management, Maastricht University Medical Center, Maastricht, The Netherlands Department of Translational Neuroscience, School for Mental Health and Neuroscience (MHeNS), University of Maastricht, The Netherlands

Department of Anesthesiology and Pain Management, Maastricht University Medical Center, Maastricht, The Netherlands

Department of Clinical Epidemiology and Medical Technology Assessment, Maastricht University Medical Center+, Maastricht, the Netherlands

Department of Anesthesiology and Pain Management, VU University Medical Center, Amsterdam, The Netherlands. To our greatest regret, Professor Perez passed away on 07.09.2017

Pain Management Center, Neurocenter of Southern Switzerland, Regional Hospital of Lugano, Via Capelli, 6962, Viganello, Switzerland

Neurocenter of Southern Switzerland, Regional Hospital of Lugano, Pain Management Center, Via Capelli, 6962, Viganello, Switzerland

Department of Anesthesiology and Pain Management, Maastricht University Medical Center+, Maastricht, The Netherlands;

Department of Translational Neuroscience, School of Mental Health and Neuroscience (MHeNS), University of Maastricht, The Netherlands

Department of Clinical Neuroscience, Karolinska Institutet, SE-171 77

Stockholm, Sweden

Muroidean Facility, School of Cardiovascular Diseases (CARIM), Maastricht, The Netherlands

Department of Translational Neuroscience, School of Mental Health and Neuroscience (MHeNS), University of Maastricht, The Netherlands 
G. Melli

M. Barbero
A. Kaelin-Lang
C. Gobbi

Laboratory for Biomedical Neurosciences, Neurocenter of Southern Switzerland, Torricella-Taverne, Switzerland; Faculty of Biomedical Sciences, Università della Svizzera Italiana, Lugano, Switzerland; Neurology Department, Neurocenter of Southern Switzerland, Lugano, Switzerland

Rehabilitation Research Laboratory 2rLab, Department of Business Economics, Health and Social Care, University of Applied Sciences and Arts of Southern Switzerland, Manno, Switzerland

Department of Neuroscience, Neurocenter of Southern Switzerland, Regional Hospital of Lugano, Switzerland

Department of Neurology, Neurocenter of Southern Switzerland, Regional Hospital of Lugano, Switzerland 
Acknowledgments 


\section{Acknowledgments}

I could not have done the work of this thesis without the support of many. Therefore, I would like to thank a number of people in particular. Firstly, I am very grateful to Prof. Roberto Perez, who to my greatest regret, is no longer with us. Roberto, I have known you from the time of my training as a resident at the department of anesthesiology and pain management at VUmc, Amsterdam. I contacted you again when I started to set up our first research projects in Lugano. From then we started up a great collaboration and it was your idea to start up a PhD program for me and Paolo, with your supervision as a co-promotor. Our collaboration gradually became more and more intense and I spoke to you on the phone every two weeks. It was you who introduced me into the wonderful world of science in pain management.. It was you who came up with the idea to combine my clinical research with experiments in animals in Maastricht, the Netherlands, in collaboration and under the supervision of Prof. dr. Bert Joosten and Prof. dr. Maarten van Kleef. Roberto, I will never forget the day that you told me about your illness. You never stopped doing research, never gave up, as research was your passion. It is incomprehensible that such a special, kind, good and wise person as you has to leave us. Thank you Roberto for sharing your pearls of wisdom with me, for your continuous support and for everything you have taught me about science in pain management. Un Fortissimo Abbraccio

My first supervisor, Prof.dr. Bert A. Joosten: Bert, thank you for giving me the opportunity to write this thesis, for the confidence in me and for the sincere and valuable guidance in the animal studies and the dissertation.

Despite the distance between Lugano and Maastricht, you were always very available. Through you I have found access to the best pain center in the world, in the most beautiful city of the Netherlands! You were also the one who brought Paolo and me into contact with Dr. Sander van Kuijk, for which I am very grateful. Bert, I hope that there are possibilities to continue this great collaboration in the future.

My second supervisor, Prof.dr. Jan A.M. van Zundert: Jan, we saw each other several times at the Swiss National Pain Congress, and I had already told you about my research projects and the collaboration with Bert in Maastricht. Eventually you became my second promoter. I would like to thank very much you for your confidence in me, for sharing expertise, and for your support in writing the dissertation. I hope that I will have the opportunity to collaborate with you in research also in the future.

My co-supervisor, Dr. Sander M.J. van Kuijk: Sander, I would like to express my sincere gratitude to for the continuous support, for your patience, encouraging motivation, and sharing your immense knowledge with me. Your contribution to all our research projects in Lugano is worth so much to us. You are always available, and incredibly kind. Is was fantastic that you came a whole week to Lugano to work on the projects and talk about future research collaboration. Thank you for the nice evenings with your wife at my place and in Maastricht. I also want to thank you for your inspiring talks and your willingness to 
continue research with us in the future. We have many projects still going on, and we know that many other projects will follow..

Dr. Paolo Maino, vice-president of the department of anesthesiology and head of the pain management center of the Neurocenter of Southern Switzerland, Regional Hospital of Lugano, my most dear colleague and best friend in Lugano: Paolo, thank you for making it possible to conduct research in our pain management center in Lugano. Thank you for your never ending enthusiasm and for the continuous encouragement. I would also like to express my great gratitude for organizing and coordinating the financial branch of animal experimental studies. Without your precious support it would not be possible to conduct this research.

My co-researchers, Glenn Franken, Jacques Debets and Lonne Heijmans, many thanks for the execution of the animal experiments and the collection of all necessary data. In particular I want to thank Glenn Franken. Glenn, I wish to express my sincere thanks to all the work you did in the context of all the animal experiments of this thesis. Thank you for your feedback and the very valuable input and comments on the manuscripts. Lonne, thank you for your great input and data-analysis in the GABA study.

Jasmin Dukanac, pain nurse and best-ever-research-coordinator: Jasmin, without your critical view, your accurateness, curiosity, great enthusiasm and enormous perseverance we would never be able to do the research at this level in our pain center in Lugano! Regarding this thesis, I would in particular like to thank you for the enormous input and work you did for the DRGS pilot study. The creation of the trial master file, the standard operating procedures, the creation of the database and insertion of the data in the database were mainly done by you. Thank you for your enormous commitment in all our clinical investigations. The future is ours!

Prof. dr. Bengh Linderoth, emeritus professor functional neurosurgery Karolinsky Instituted; adj. prof., dept physiology Oklahoma University, USA. You were involved with all three animal studies and your intellectual input brought our manuscripts to a higher level. Although we were working from different countries, Sweden, Switzerland, the Netherlands, you were always very available and responded always immediately to all my questions. It has been a great honor for me to collaborate with you.

Giorgia Melli, neurologist at the Neurocenter of Southern Switzerland, Regional Hospital of Lugano: Cara Giorgia, thank you for your help regarding neurologic part of the DRGS pilot study. Thank you also for the recruitment of patients in this project. Our collaboration in this project has been hopefully only the start of many other future projects together.

Prof. Dr.med. Kaelin-Lang, medical and scientific director of the neurocenter of Southern Switzerland, Regional Hospital of Lugano, and Prof. Dr.med. Gobbi, co-head of the department of neurology of the neurocenter of Southern Switzerland, Regional Hospital of Lugano: thank you both for your input and the collaboration in the DRGS for polyneuropathy project. Thank you professor Kaelin for your support regarding all our 
other research activities at the Pain Management Center of the Regional Hospital of Lugano.

Prof. Marco Barbero, professor, docent, researcher and physiotherapist: Thank you Marco for your participation in the DRGS pilot study and your assistance with the data analysis of the pain extent part. More research in collaboration with you will definitely follow.

The members of the defense committee; thank you for your critical review of this thesis, I feel honored you are willing to be present at my dissertation. In particular I want to thank Prof.dr. Maarten van Kleef, who was initially my co-promoter, but in the end became a member of the defense committee. Thank you for the stimulating discussions, your openness, directness, humor and critical eye. With all your that you achieved in pain research you have my sincere gratitude and deep respect.

Gerard Barendse, Lysandre Berry, Jeroen Steenhuizen en Jasper Kal (OLVG): although you were not directly involved with this thesis, you introduced me in the world of pain management and stimulated my curiosity. The fellowship in pain management at your site was of a very high level, and I will carry all your tips and tricks for my whole life in pain management with me.

Vanessa Ross and Camille Remy: my colleagues at the Pain Management Center, Neurocenter of Southern Switzerland, Regional Hospital of Lugano: Dear Vanessa and Camille, thank you for your great collaboration and you help with the recruitment of patients in our studies.

Guigne Kooijman, Irene van Riel, and Dr. Reineke A. Schoot, my buddies during medicine study: thank you for being such a great friends. Reineke, thank you for all your advices, and advices with the making of the final dissertation.

Prof. Hemme Batjes and Juliette Batjes + children: thank you for your precious friendship and support in difficult times. Thank you Hemme, for explaining me the important difference between 'urgent' and 'important', when I just didn't know anymore where to start.. Your advices helped me a lot!

Dear family, Jelle, Zico and Mika. Thank for your support and love which always gives me energy to keep on going. Dear Tiemen, father, thank you for your many visits to us from the Netherlands. You always taught me to be critical, honest, to look further, and to never give up. Dear Winifred, mother, you have provided me moral and emotional support in my life. I wish you were still here with us. I am also grateful to all my other family members (including the family of Jelle of course): you were always keen to know what I was doing and how I was proceeding. Thanks for all your encouragements. 


\section{About the author}

Eva Koetsier was born on November 15, 1976 in Monnickendam, a little town in the Netherlands, very close to Amsterdam. She first graduated in Law in 2001 and later in Medicine in 2005 at the University of Amsterdam. During the years of study in Medicine, from 2001 to 2003, she was a legal consultant for the department of labor law, at an eminent law firm in Amsterdam in the Netherlands. From 2005 to 2006, during her pregnancy of her first son, she worked as resident in the emergency room of cardiology at the BovenlJ Hospital in Amsterdam. She started her training in anesthesiology in the VU University Medical Center, Amsterdam, the Netherlands (head S. Loer) in 2006. During this period she also worked as a resident in training for one year at the Lucas Andreas Hospital, Amsterdam, and one year the intensive care unit of the VU University Medical Center. In 2011 she was registered as anesthesiologist. From 2011-2013 she worked as anesthesiologist at the OLVG Hospital in Amsterdam, the biggest regional hospital of Amsterdam, located in the city center. Her knowledge of law has allowed her to be appointed member of the legal committee of the National Association of Anesthesia in the Netherlands from 1010 to 2016 and to be editor of a medico-legal column in the journal of this association. In 2012, at the end of a one-year fellowship at the OLVG Hospital in Amsterdam, she obtained the title of specialty in Interventional Pain Therapy. Since 2013 she has been working as an anesthesiologist and pain specialist at the Pain Management Center of the Neurocenter of Southern Switzerland (NSI) and in the Anesthesiology department of the Lugano Regional Hospital. In Lugano, she has developed a scientific research activity in the context of several innovative therapies for chronic pain. She married to Jelle Jansen and they have two sons: Zico (2005) and Mika (2010). 



\section{List of Publications}

\section{Publications related to this thesis in peer-reviewed journals}

- Koetsier E, van Kuijk SMJ, Melli G, Dukanac J, Barbero M, van Zundert J, Joosten EA, Maino P. Dorsal Root Ganglion Stimulation for the Management of Intractable Painful Polyneuropathy: A prospective pilot study. Submitted.

- Koetsier E, Franken G, Debets J, van Kuijk SMJ, Linderoth B, Joosten EA, Maino P. Dorsal Root Ganglion Stimulation in Experimental Painful Diabetic Polyneuropathy: Delayed Wash-out of Pain Relief after Low-Frequency $(1 \mathrm{~Hz})$ Stimulation. Neuromodulation. 2019;23:177-184.

- $\quad$ Koetsier E, Franken G, Debets J, Heijmans L, van Kuijk SMJ, Linderoth B, Joosten EA, Maino P. Mechanism of Dorsal Root Ganglion Stimulation for Pain Relief in Painful Diabetic Polyneuropathy is Not Dependent on GABA Release in the Dorsal Horn of the Spinal Cord. CNS Neuroscience \& Therapeutics. 2020;26:136143.

- $\quad$ Koetsier E, Franken G, Debets J, van Kuijk SMJ, Perez RSGM, Linderoth B, Joosten EA, Maino P. Effectiveness of Dorsal Root Ganglion Stimulation and Dorsal Column Spinal Cord Stimulation in a Model of Experimental Painful Diabetic Polyneuropathy. CNS Neuroscience \& Therapeutics. 2019;25:367-374.

- Koetsier E, Boer Ch, Loer, SA, Complaints and incident reports related to anaesthesia service are foremost attributed to nontechnical skills. European Journal of Anaesthesiology 2011;28:29-33

- Maino P, Koetsier E, Kaelin-Lang A, Gobbi C, Perez RSGM. Efficacious Dorsal Root Ganglion Stimulation for Painful Small Fiber Neuropathy: A Case Report. Pain Physician, 2017;20:E459-E463

\section{Other publications in peer reviewed journals}

- Croci DM, Koetsier E, Maino P, Reinert M. Cervical radiculopathy caused by Vertebral Artery Loop: Is a focused fluoroscopic-guided cervical epidural steroid injection a possible treatment modality? Pain Practice. Accepted 14.04.2020. Online ahead of print 
- $\quad$ Barbero M, Schneebelia A, Koetsier E, Maino P. Myofascial pain syndrome and trigger points: evaluation and treatment in patients with musculoskeletal pain Curr Opin Support Palliat Care. 2019;13:270-276

- Maino P, Koetsier E. Reply to Letter to the Editor Regarding Sonographic Assistance for Intrathecal Drugs Delivery System Refill. Pain Pract. 2019;19:677

- Maino P, van Kuijk SMJ, Koetsier E. Refill Port Identification of Intrathecal Drug Delivery System Devices with a Raised Fill Port, Pain Practice 2019;19:418-425

- Koetsier E, Maino P. Commentary on: Safe Use of Epidural Corticosteroid Injections: Recommendations of the WIP Benelux Work Group, by Van Boxem et al., Pain Practice 2019;19:344

- $\quad$ Maino P, van Kuijk SMJ, Perez RSGM, Koetsier E. Refill Procedures of Intrathecal Drug Delivery Systems with a Recessed Fill Port on the Pump Surface: a Prospective Comparison Study of Ultrasound-Guided vs. Blind Refill Technique. Neuromodulation. 2019;22:799-804

- Maino P, Presilla S, Colli Franzone PA, van Kuijk SMJ, Perez RSGM, Koetsier E. Radiation Dose Exposure for Lumbar Transforaminal Epidural Steroid Injections and Facet Joint Blocks under CT versus Fluoroscopic Guidance. Pain Pract, 2018;18: 798-804

- $\quad$ Maino P, van Kuijk SMJ, Perez RSGM, Koetsier E. Ease of Fill Port Access During the Ultrasound-Guided vs. the Blind Refill Technique of Intrathecal Drug Delivery Systems with a Raised Septum, a Prospective Comparison Study. Neuromodulation. 2018;21:641-647

- Maino P, Perez RSGM, Koetsier E. Intrathecal Pump Refills, Pocket Fills, and Symptoms of Drug Overdose: A Prospective, Observational Study Comparing the Injected Drug Volume vs. the Drug Volume Effectively Measured Inside the Pump. Neuromodulation. 2017;20:733-739

- Koetsier E, Kallewaard JW, Maino P. Commentary on: Does provocative discography cause clinically important injury to the lumbar intervertebral disc? A 10-year matched cohort study, by Cueller et al. Spine J. 2017;17:610-611

- Maino P, Koetsier E, Perez RSGM. The Accuracy of Template-Guided Refill Technique of Intrathecal Pumps Controlled by Fluoroscopy: An Observational Study. Neuromodulation. 2015;18:428-432

- Koetsier E, Maino P, (shared first authorship), Perez RSGM. Fentanyl Overdose Caused by Malfunction of SynchroMed II Intrathecal Pump: Two Case Reports. Reg Anesth Pain Med. 2014;39:434-437

- Koetsier E, Boer Ch, Loer, SA, Complaints and incident reports related to anaesthesia service are foremost attributed to nontechnical skills. European Journal of Anaesthesiology 2011;28:29-33

- Baldeschi L, MacAndie K, Koetsier E, Blank LE, Wieringa WM, The influence of previous orbital irradiation on the outcome of rehabilitative decomplression surgery in graves orbitopathy. Am J Ophtalmol. 2008; 145: 534-540 


\section{Other publications (medical law)}

- Koetsier, E Garmers, L; 2010- 2015: Regular column about law and anesthesiologists in a magazine of Anethesiology in the Netherlands

- $\quad$ Garmers L; Koetsier E; Arts vogelvrij op internet?, De Anesthesioloog 2010;4:14

- Koetsier E; Jap-A-Joe K, Veilig melden, beroepsgeheim en verschoningsrecht versus waarheidsvinding (Safe reporting, confidentiality and privilege versus truth), de anesthesioloog 2010;3:6-9. 




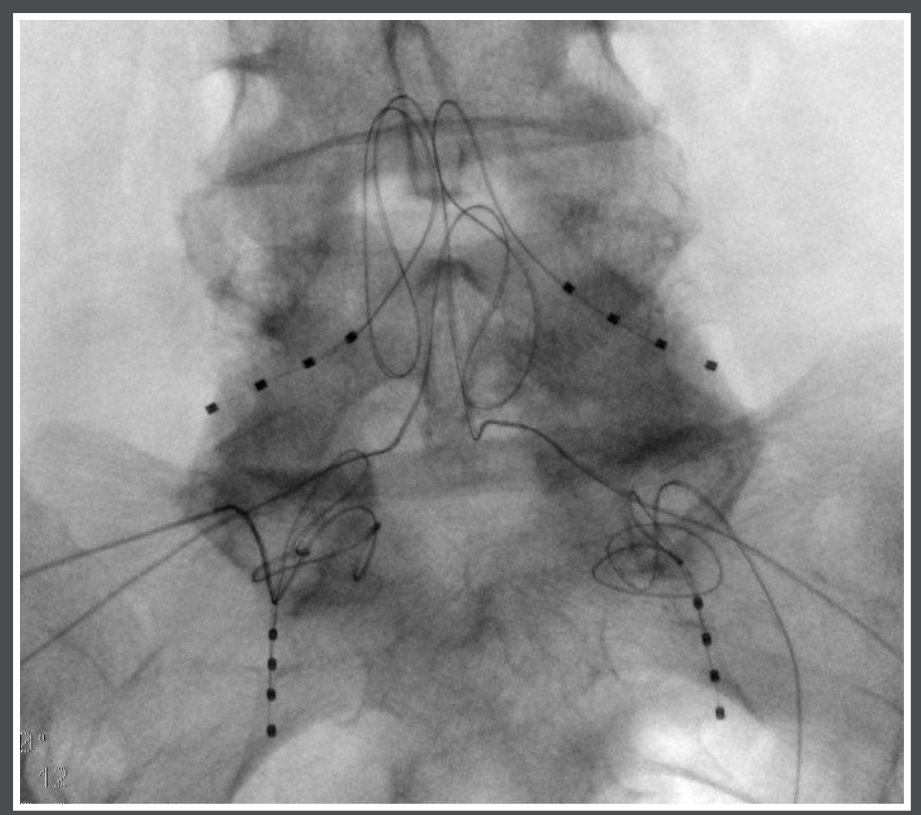

